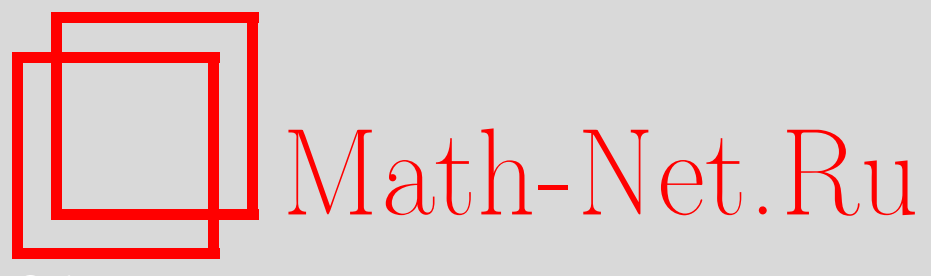

С. Д. Глызин, А. Ю. Колесов, Н. Х. Розов, Релаксационные автоколебания в сетях импульсных нейронов, $У М H$, 2015, том 70, выпуск 3, 3-76

DOI: https://doi.org/10.4213/rm9659

Использование Общероссийского математического портала Math-Net.Ru подразумевает, что вы прочитали и согласны с пользовательским соглашением http://www . mathnet.ru/rus/agreement

Параметры загрузки:

IP : 35.174 .16 .151

26 апреля 2023 г., 16:02:51

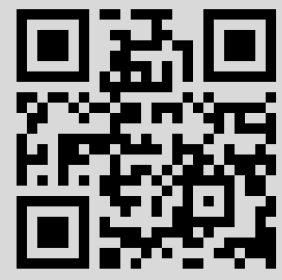


УДК 517.926

\title{
Релаксационные автоколебания \\ в сетях импульсных нейронов
}

\author{
С. Д. Глызин, А. Ю. Колесов, Н. Х. Розов
}

Статья посвящена проблеме математического моделирования нейронной активности. Предлагаются новые классы сингулярно возмущенных дифференциально-разностных уравнений с запаздыванием вольтерровского типа, с помощью которых описывается функционирование как отдельного нейрона, так и нейронных сетей с различным характером связи (электрическим и химическим). Развиваются специальные асимптотические методы, позволяющие изучить вопросы о существовании и устойчивости в указанных системах релаксационных периодических движений.

Библиография: 56 названий.

Ключевые слова: нейронные модели, дифференциально-разностные уравнения, релаксационные колебания, асимптотика, устойчивость, буферность, bursting-эффект.

DOI: $10.4213 / \mathrm{rm} 9659$

\section{СОДЕРЖАНИЕ}

1. Основные принципы математического моделирования в нейродинамике 4

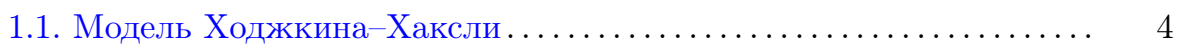

1.2. Двумерные модели .................................. 8

1.3. Трехмерные модели . . . . . . . . . . . . . . . . . . . . 10

1.4. Модель Хопфилда с запаздыванием .................... 16

1.5. Основные способы моделирования нейронных сетей.......... 17

1.6. Новый подход к моделированию отдельного нейрона.......... 18

2. Математическая модель импульсного нейрона

с одним запаздыванием.......................... 21

2.1. Основной результат ........................... 21

2.2. Существование релаксационного цикла................ 24

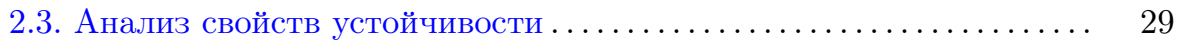

Работа выполнена при поддержке РФФИ (грант № 15-01-04066-а) и проекта 1875 госзадания на НИР № 2014/258.

(C) С. Д. Глызин, А. Ю. Колесов, Н. Х. Розов, 2015 
3. Автоволновые процессы в сетях связанных нейронов .............. 32

3.1. Базовые теоремы ............................. 32

3.2. Аттракторы предельного отображения ................. 38

3.3. Случай кольцевой однонаправленной связи.............. 46

3.4. Случай полносвязной нейронной сети ................. 51

3.5. Об одном способе математического моделирования химических

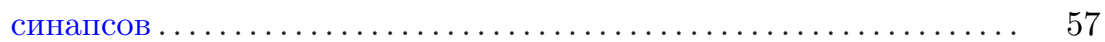

4. Модель импульсного нейрона с двумя запаздываниями ............ 59

4.1. Математическое описание bursting-эффекта............... 59

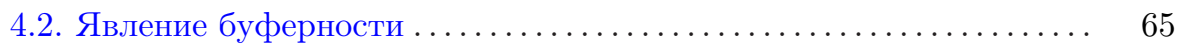

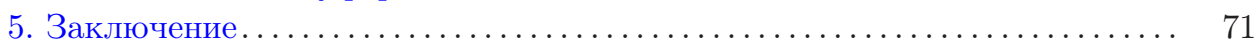

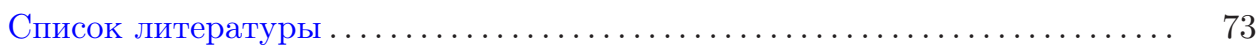

\section{1. Основные принципы математического моделирования в нейродинамике}

1.1. Модель Ходжкина-Хаксли. Ниже дается краткий обзор основных идей, лежащих в основе математического моделирования нейродинамических процессов, и приводится описание некоторых наиболее известных непрерывных нейронных моделей, представляющих собой системы обыкновенных дифференциальных уравнений или дифференциально-разностных уравнений с запаздыванием. Основная цель статьи - анализ новой математической модели функционирования отдельного нейрона, учитывающей эффекты запаздывания.

Отметим, что нас будут интересовать так называемые импульсные нейроны, мембранные потенциалы которых претерпевают кратковременные всплески (в нейродинамике их называют спайками), чередующиеся с относительно спокойными участками. Для того чтобы в последующем дать математическое описание спайков, остановимся сначала на биофизическом механизме их возникновения.

Как известно, электрические процессы в нейронах носят ионный характер (см. работы [1]-[3] и содержащуюся в них библиографию). При этом мембрана клетки выполняет роль изолятора, поскольку обладает высоким электрическим сопротивлением, большой электроемкостью и малой, причем избирательной, проницаемостью. Далее, существуют специальные каналы, через которые в соответствии со своей концентрацией и электрическим зарядом ионы проникают сквозь мембрану (это так называемый пассивный транспорт, не требующий затрат энергии). Добавим еще, что для каждого вида ионов имеется свой тип таких каналов.

Пассивный транспорт, состоящий в основном из ионов натрия $\left(\mathrm{Na}^{+}\right)$и калия $\left(\mathrm{K}^{+}\right)$, приводит к тому, что в процессе движения ионов положительный и отрицательный заряды накапливаются на противоположных сторонах поверхности мембраны. В результате создается мембранный потенциал, равный разности потенциалов между внутренней и наружной ее сторонами. А так как в состоянии покоя мембрана хорошо проницаема извне для ионов калия и менее проницаема для ионов натрия, то внутриклеточная среда имеет высокую концентрацию $\mathrm{K}^{+}$, а межклеточное пространство - высокую концентрацию $\mathrm{Na}^{+}$. 
При этом суммарный электрический заряд внутри клетки оказывается меньше внешнего заряда. Тем самым, в состоянии покоя мембрана сильно поляризована, а ее потенциал отрицателен.

Дополнительным механизмом поляризации, обеспечивающим электроотрицательность внутренней среды клетки, служит так называемый активный транспорт: перенос ионов натрия изнутри наружу за счет энергии АТФ (аденозинтрифосфорной кислоты).

Разнонаправленные ионные потоки, связанные с пассивным и активным транспортом, могут уравновешивать друг друга, и в этом случае состояние покоя устойчиво. В случае же импульсного нейрона оно теряет устойчивость, а мембранный потенциал переходит в режим генерации спайков.

Процесс формирования спайка можно разбить на два этапа. Первый этап начинается с открытия в мембране натриевых каналов и поступления через них ионов $\mathrm{Na}^{+}$внутрь клетки. Положительный заряд этих ионов снижает отрицательный заряд внутриклеточного вещества, и происходит частичная деполяризация мембраны. Причем, что характерно, по времени данный этап развивается лавинообразно: чем сильнее деполяризация мембраны, тем больше открывается натриевых каналов, что сопровождается резким скачком мембранного потенциала. В итоге последний достигает своего положительного максимума.

Второй этап, также развивающийся лавинообразно, в определенном смысле противоположен первому. А именно, положительность мембранного потенциала в точке максимума приводит к одновременному уменьшению проницаемости мембраны изнутри наружу для ионов натрия и к увеличению аналогичной проницаемости для ионов калия. В результате последние начинают покидать клетку, унося положительный заряд, что приводит к резкому падению мембранного потенциала. Более того, отрицательный заряд на внутренней поверхности мембраны увеличивается и достигает значения, превышающего по абсолютной величине соответствующее значение в состоянии покоя. Иными словами, происходит гиперполяризация клетки.

Сразу после спайка нервная клетка находится в состоянии рефрактерности, которое характеризуется полной или относительной невосприимчивостью к внешнему воздействию. В этот период при помощи ионного транспорта значения концентраций ионов и мембранного потенциала медленно восстанавливаются до значений, соответствующих положению равновесия. По прошествии указанного периода нейрон возвращается в первоначальное состояние и вновь обретает способность генерировать спайки.

Описанное поведение мембранного потенциала нейрона, характеризующееся наличием сменяющих друг друга участков быстрых и медленных движений, удивительным образом напоминает электрические релаксационные колебания в различных автогенераторах. Именно по этой причине подавляющее большинство нейронных математических моделей базируется на так называемой гипотезе об эквивалентности. В рамках данной гипотезы мы заменяем биологический нейрон физическим объектом - некоторым генератором с сосредоточенными электрическими параметрами. В свою очередь, упомянутый генератор моделируется нелинейной системой обыкновенных дифференциальных уравнений 
или аналогичной системой с запаздыванием. А так как колебания мембранного потенциала носят заведомо релаксационный характер, то получающаяся в итоге система, как правило, оказывается сингулярно возмущенной.

Одна из первых непрерывных нейронных моделей была предложена Ходжкиным и Хаксли в известной работе [4] (см. также [2], [5], [6]). Рассматриваемая ими модель учитывала функционирование "натриево-калиевого насоса" и опиралась на гипотезу об эквивалентности. Точнее говоря, в [4] были сделаны следующие формальные допущения.

ГиПотезА 1 (об эквивалентности). Считаем, что биологический нейрон эквивалентен генератору, блок-схема которого показана на рис. 1.

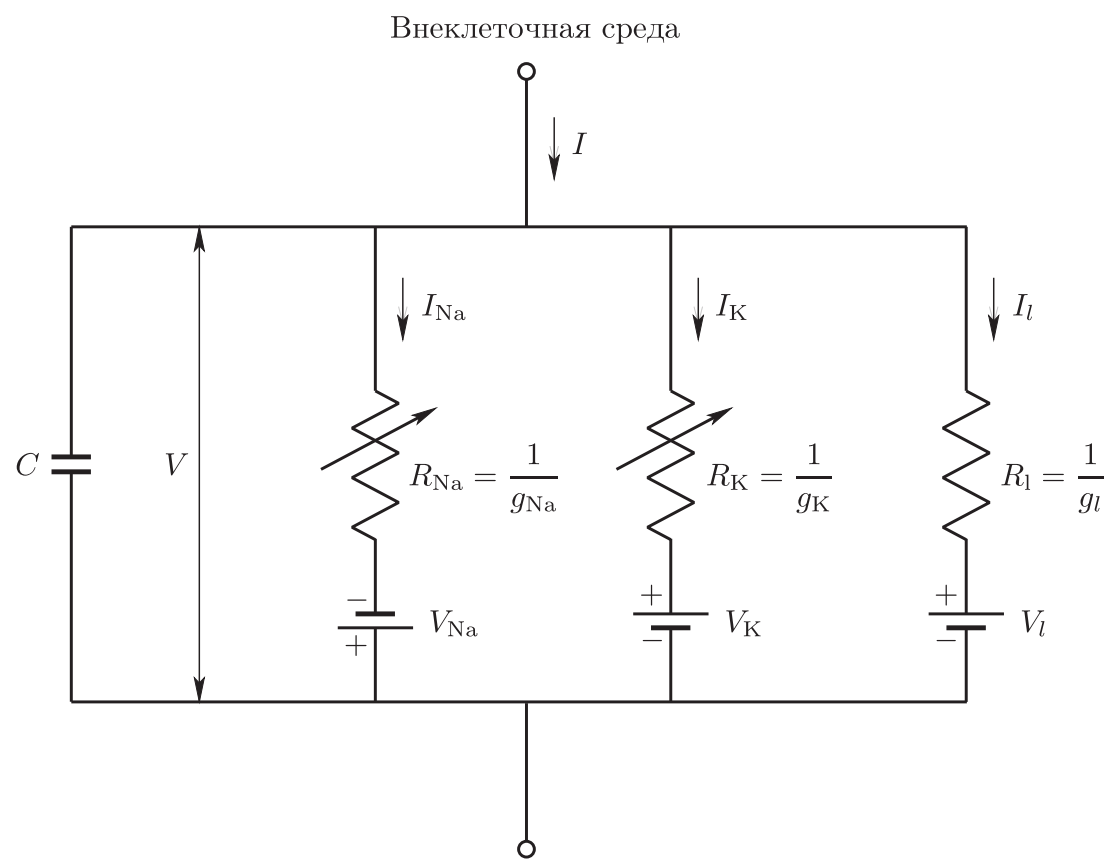

Внутриклеточная среда

Рис. 1

Приведенный рисунок нуждается в некоторых пояснениях. Отметим, во-первых, что положительное направление токов на нем соответствует направлению внутрь клетки. Во-вторых, справедливы равенства

$$
V(t)=E(t)-E_{r}, \quad V_{\mathrm{Na}}=E_{\mathrm{Na}}-E_{r}, \quad V_{\mathrm{K}}=E_{\mathrm{K}}-E_{r}, \quad V_{l}=E_{l}-E_{r},
$$

где $E(t)$ - мембранный потенциал, $E_{r}$ - абсолютное значение потенциала покоя, а через $E_{\mathrm{Na}}, E_{\mathrm{K}}, E_{l}$ обозначены некоторые равновесные (не зависящие от $t$ ) значения натриевого потенциала, калиевого потенциала и потенциала утечки соответственно. В-третьих, величина $C$ представляет собой мембранную емкость на единицу площади и предполагается постоянной. И наконец, в-четвертых, 
считаем, что натриевая и калиевая проводимости $g_{\mathrm{Na}}=g_{\mathrm{Na}}(t), g_{\mathrm{K}}=g_{\mathrm{K}}(t)$ зависят от $t$, а проводимость утечки $\bar{g}_{l}$ постоянна.

ГиПотезА 2. Имеет место равенство

$$
g_{\mathrm{K}}=\bar{g}_{\mathrm{K}} n^{4}
$$

где $\bar{g}_{\mathrm{K}}=\mathrm{const}>0$, a $n=n(t) \in(0,1)$. Предполагаем, что функиия $n(t)$, представляющая собой вероятностную характеристику активации калиевых каналов, удовлетворяет уравнению

$$
\dot{n}=\alpha_{n}(V)(1-n)-\beta_{n}(V) n .
$$

Здесъ $\alpha_{n}(V), \beta_{n}(V)$ - некоторье непрерьвные при всех $V \in \mathbb{R}$ и строго положительные функиии.

ГиПотезА 3. Для натриевой проводимости справедливо равенство

$$
g_{\mathrm{Na}}=\bar{g}_{\mathrm{Na}} m^{3} h, \quad \bar{g}_{\mathrm{Na}}=\text { const }>0,
$$

где $m(t) \in(0,1), h(t) \in(0,1)$ - вероятностные характеристики активации и инактивации натриевых каналов. Эти характеристики удовлетворяют системе дифференииальных уравнений

$$
\dot{m}=\alpha_{m}(V)(1-m)-\beta_{m}(V) m, \quad \dot{h}=\alpha_{h}(V)(1-h)-\beta_{h}(V) h,
$$

где, как и в (1.2), функции $\alpha_{m}(V), \beta_{m}(V), \alpha_{h}(V), \beta_{h}(V)$ непрерьвны на всей оси и положительны.

Основой модели Ходжкина-Хаксли служат законы Ома и Кирхгофа, приводящие к равенствам (см. рис. 1)

$$
\begin{gathered}
I=C \dot{V}+I_{\mathrm{Na}}+I_{\mathrm{K}}+I_{l}, \quad I_{\mathrm{Na}}=g_{\mathrm{Na}}\left(V-V_{\mathrm{Na}}\right), \\
I_{\mathrm{K}}=g_{\mathrm{K}}\left(V-V_{\mathrm{K}}\right), \quad I_{l}=\bar{g}_{l}\left(V-V_{l}\right) .
\end{gathered}
$$

Здесь $I$ - суммарный мембранный ток, предполагающийся постоянным, а через $I_{\mathrm{Na}}, I_{\mathrm{K}}, I_{l}$ обозначены натриевый ток, калиевый ток и ток утечки соответственно. Далее, учитывая в (1.5) соотношения (1.1)-(1.4), окончательно получаем:

$$
\begin{gathered}
C \dot{V}=\bar{g}_{\mathrm{K}} n^{4}\left(V_{\mathrm{K}}-V\right)+\bar{g}_{\mathrm{Na}} m^{3} h\left(V_{\mathrm{Na}}-V\right)+\bar{g}_{l}\left(V_{l}-V\right)+I, \\
\dot{n}=\frac{n_{\infty}(V)-n}{\tau_{n}(V)}, \quad \dot{m}=\frac{m_{\infty}(V)-m}{\tau_{m}(V)}, \quad \dot{h}=\frac{h_{\infty}(V)-h}{\tau_{h}(V)},
\end{gathered}
$$

где

$$
\begin{array}{ll}
n_{\infty}(V)=\frac{\alpha_{n}(V)}{\alpha_{n}(V)+\beta_{n}(V)}, & \tau_{n}(V)=\frac{1}{\alpha_{n}(V)+\beta_{n}(V)}, \\
m_{\infty}(V)=\frac{\alpha_{m}(V)}{\alpha_{m}(V)+\beta_{m}(V)}, & \tau_{m}(V)=\frac{1}{\alpha_{m}(V)+\beta_{m}(V)}, \\
h_{\infty}(V)=\frac{\alpha_{h}(V)}{\alpha_{h}(V)+\beta_{h}(V)}, & \tau_{h}(V)=\frac{1}{\alpha_{h}(V)+\beta_{h}(V)} .
\end{array}
$$


Добавим еще, что в системе (1.6), (1.7) параметры $\bar{g}_{\mathrm{K}}, \bar{g}_{\mathrm{Na}}, \bar{g}_{l}, C$ по своему физическому смыслу положительны, а знаки постоянных $V_{\mathrm{K}}, V_{\mathrm{Na}}, V_{l}, I$ произвольны.

В работах Ходжкина и Хаксли в качестве функций $\alpha_{n}, \beta_{n}, \alpha_{m}, \beta_{m}, \alpha_{h}, \beta_{h}$ изначально были взяты

$$
\begin{array}{lll}
\alpha_{n}=0.01(V+10)\left[\exp \left(\frac{V+10}{10}\right)-1\right]^{-1}, & \beta_{n}=0.125 \exp \left(\frac{V}{80}\right), \\
\alpha_{m}=0.1(V+25)\left[\exp \left(\frac{V+25}{10}\right)-1\right]^{-1}, & \beta_{m}=4 \exp \left(\frac{V}{18}\right) \\
\alpha_{h}=0.07 \exp \left(\frac{V}{20}\right), & \beta_{h}=\left[\exp \left(\frac{V+30}{10}\right)+1\right]^{-1} .
\end{array}
$$

Уточнение этих функций выполнялось и позднее. Для примера сошлемся на работу [7], где приведен вид этих функций и набор параметров $\bar{g}_{\mathrm{K}}, \bar{g}_{\mathrm{Na}}, \bar{g}_{l}$, $C, V_{\mathrm{K}}, V_{\mathrm{Na}}, V_{l}$, при которых модель $(1.6),(1.7)$ хорошо описывает прохождение нервных импульсов по аксонам гигантского кальмара (параметр $I$ в этом случае был управляющим и менялся на некотором промежутке).

1.2. Двумерные модели. Теоретический анализ модели Ходжкина-Хаксли (1.6), (1.7) довольно затруднителен, и обычно ее изучают численно. Для аналитического же исследования автоколебаний в изолированном нейроне привлекаются различные упрощенные модели, учитывающие лишь внешнее проявление активности нейрона (изменение мембранного потенциала и генерации спайков) и не отражающие внутриклеточных причин этих изменений.

В качестве упрощенных нейронных моделей широко используются двумерные сингулярно возмущенные системы вида

$$
\varepsilon \dot{u}=f(u, v), \quad \dot{v}=g(u, v),
$$

где $u=u(t)$ - мембранный потенциал, $v=v(t)$ имеет смысл силы тока, $\varepsilon>0$ малый параметр. Принимая во внимание релаксационный характер электрической активности клетки, будем считать, что на правые части $f, g \in C^{\infty}$ наложены стандартные ограничения [8], обеспечивающие существование устойчивого релаксационного цикла.

Некоторые модели вида (1.8) выводятся из системы (1.6), (1.7) при ряде дополнительных предположений. Другие же выписываются независимо. К последним относится известная система Фитцхью-Нагумо [9]-[12], имеющая вид

$$
\varepsilon \dot{u}=v+u-\frac{u^{3}}{3}+c, \quad \dot{v}=a-u-b v,
$$

где $0<\varepsilon \ll 1, a, b=$ const $>0, c=$ const $\in \mathbb{R}$.

В работе [11] система (1.9) использовалась в качестве феноменологической модели, воспроизводящей основные свойства волн возбуждения в более сложной модели (1.6), (1.7). Далее, в статье [12] было показано, что та же самая система уравнений описывает электрические колебания в генераторе с туннельным диодом (см. рис. 2) при условии, что вольт-амперная характеристика последнего (см. рис. 3) аппроксимирована кубической параболой. И наконец, 


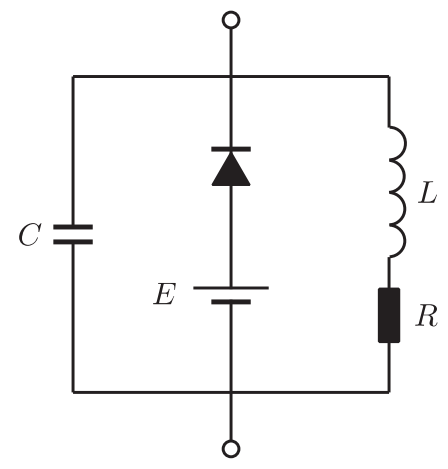

Рис. 2

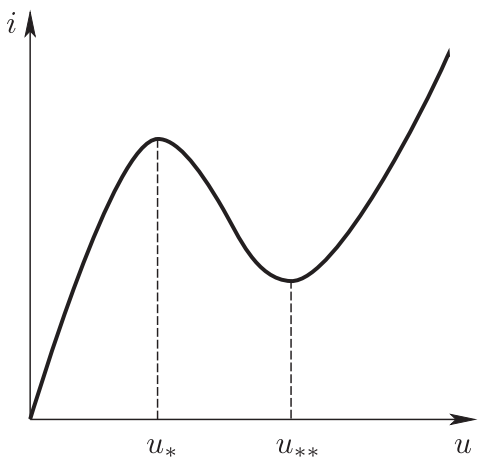

Рис. 3

следует отметить, что при соответствующем выборе параметров система (1.9) допускает устойчивый релаксационный цикл.

В класс моделей (1.8) попадает также известная система Морриса-Лекаpa [13]

$$
\begin{gathered}
C \dot{V}=\bar{g}_{\mathrm{Ca}} m_{\infty}(V)\left(V_{\mathrm{Ca}}-V\right)+\bar{g}_{\mathrm{K}} n\left(V_{\mathrm{K}}-V\right)+\bar{g}_{l}\left(V_{l}-V\right)+I, \\
\dot{n}=\lambda_{n}(V)\left(n_{\infty}(V)-n\right) .
\end{gathered}
$$

Здесь, как и в случае системы $(1.6),(1.7)$, постоянные $C, \bar{g}_{\text {Са }}, \bar{g}_{\mathrm{K}}, \bar{g}_{l}$ положительны, а знаки параметров $V_{\mathrm{Ca}}, V_{\mathrm{K}}, V_{l}, I$ произвольны. Что же касается функций $m_{\infty}(V), \lambda_{n}(V), n_{\infty}(V)$, то они задаются равенствами

$$
\begin{gathered}
m_{\infty}(V)=\frac{1}{2}\left(1+\operatorname{th} \frac{V-V_{1}}{V_{2}}\right), \quad n_{\infty}(V)=\frac{1}{2}\left(1+\operatorname{th} \frac{V-V_{3}}{V_{4}}\right), \\
\lambda_{n}(V)=\lambda \operatorname{ch} \frac{V-V_{3}}{2 V_{4}},
\end{gathered}
$$

где $V_{j}=$ const $\in \mathbb{R}, j=1, \ldots, 4, \lambda=$ const $>0$. Добавим еще, что в случае $(1.10),(1.11)$ мы имеем дело с электросхемой, показанной на рис. 1, в которой натриевый ток заменен на кальциевый.

При условии $\lambda \gg 1$ после нормировки времени и переобозначения переменных система (1.10) преобразуется к виду (1.8) при $\varepsilon=1 / \lambda$. Более того, можно показать, что при подходящем выборе параметров у нее существует устойчивый релаксационный цикл.

Еще одним примером системы (1.8) служит двумерный вариант модели Хиндмарша и Роуз [14], [15], имеющий вид

$$
\varepsilon \dot{u}=v-a u^{3}+b u^{2}+I, \quad \dot{v}=c-d u^{2}-v .
$$

Обычно система (1.12) рассматривается при фиксированных значениях параметров $\varepsilon=1, a=1, b=3, c=1, d=5, I=2.7$. Если же при указанных значениях $a, b, c, d, I$ уменьшать $\varepsilon$, то ее устойчивый цикл становится релаксационным. 
Двумерные модели (1.8) обладают по крайней мере двумя существенными недостатками. Первый недостаток состоит в том, что реализующиеся в этих моделях классические релаксационные колебания не вполне адекватны колебаниям мембранного потенциала реального нейрона. В принципе, данный недостаток может быть исправлен. Например, в работах [16], [17] предложена некоторая модификация модели (1.9), в которой колебания компоненты $u=u(t)$ носят $\delta$-образный характер. Такие колебания в большей степени соответствуют реальности.

Вторым недостатком является тот факт, что модели (1.8) не охватывают важное нейродинамическое явление, называемое bursting-эффектом. Этот феномен заключается в чередовании пакетов импульсов (наборов из нескольких подряд идущих интенсивных всплесков) с участками относительно спокойного изменения мембранного потенциала. По всей видимости, добиться bursting-эффекта в двумерных моделях невозможно.

1.3. Трехмерные модели. Изучению "bursting behavior" посвящена обширная литература (см., например, [3], [18]-[24] и приведенную в этих работах библиографию). Как правило, для математического моделирования данного феномена привлекаются трехмерные сингулярно возмущенные системы вида

$$
\varepsilon \dot{x}=f(x, y), \quad \dot{y}=g(x, y), \quad x=\left(x_{1}, x_{2}\right) \in \mathbb{R}^{2}, \quad y \in \mathbb{R}, \quad 0<\varepsilon \ll 1,
$$

в которых при определенных ограничениях на правые части $f, g \in C^{\infty}$ реализуются незатухающие релаксационные автоколебания с bursting-эффектом.

Для пояснения сути дела приведем простейший набор условий, гарантирующих существование у системы (1.13) устойчивого bursting-цикла.

Остановимся сначала на совокупности ограничений, связанных с так называемой системой быстрых движений

$$
\frac{d x}{d \tau}=f(x, y), \quad y \in \mathbb{R},
$$

где $\tau=t / \varepsilon$, а переменная $y$ рассматривается как параметр. Будем предполагать, что уравнение $f(x, y)=0$, из которого определяются состояния равновесия этой системы, задает в $\mathbb{R}^{3}$ гладкую кривую, распадающуюся на три части (см. рис. 4):

$$
\begin{gathered}
\left\{(x, y): x=\varphi_{1}(y),-\infty<y \leqslant y_{3}\right\}, \quad\left\{(x, y): x=\varphi_{2}(y), y_{1} \leqslant y \leqslant y_{3}\right\}, \\
\left\{(x, y): x=\varphi_{3}(y), y_{1} \leqslant y<+\infty\right\} .
\end{gathered}
$$

Здесь $y_{1}<y_{3}$, а вектор-функции $\varphi_{j}(y), j=1,2,3$, таковы, что

$$
\begin{gathered}
\varphi_{1}(y) \in C^{\infty}\left(-\infty, y_{3}\right), \quad \varphi_{2}(y) \in C^{\infty}\left(y_{1}, y_{3}\right), \quad \varphi_{3}(y) \in C^{\infty}\left(y_{1},+\infty\right), \\
\varphi_{1}\left(y_{3}\right)=\varphi_{2}\left(y_{3}\right), \quad \varphi_{2}\left(y_{1}\right)=\varphi_{3}\left(y_{1}\right) .
\end{gathered}
$$

Для формулировки следующей серии ограничений введем в рассмотрение двумерные матрицы $A_{j}(y)=\left.f_{x}^{\prime}(x, y)\right|_{x=\varphi_{j}(y)}, j=1,2,3$. Считаем, что при всех $-\infty<y<y_{3}$ матрица $A_{1}(y)$ гурвицева, а при $y=y_{3}$ одно ее собственное значение становится равным нулю (другое отрицательно). Далее, обозначим через $e_{0}$ 


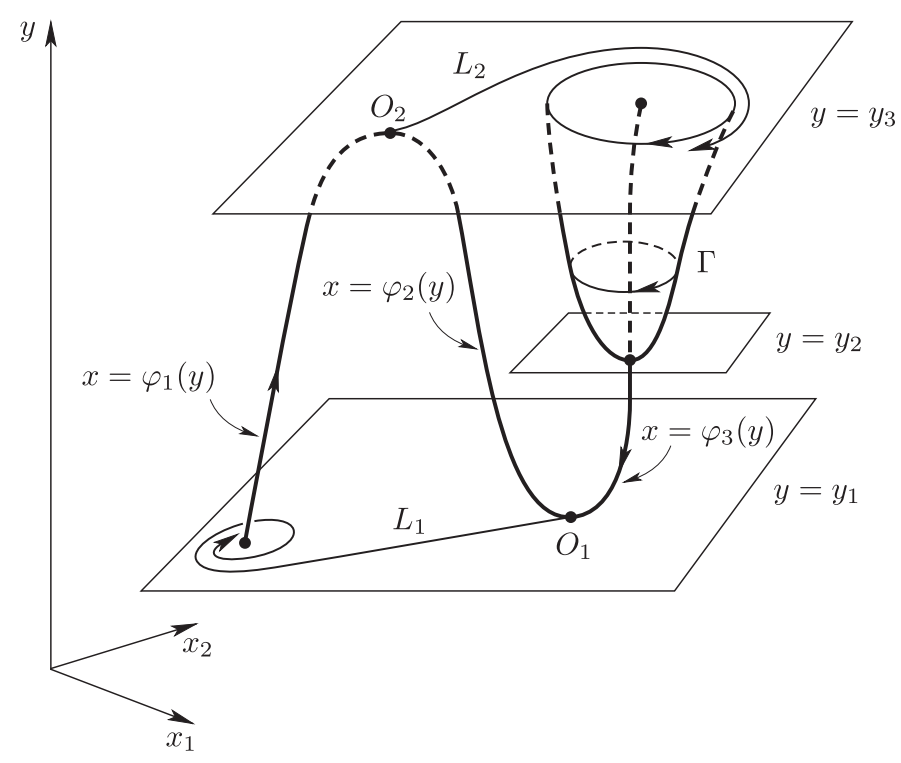

Рис. 4

собственный вектор матрицы $A_{1}^{T}\left(y_{3}\right)$ (здесь $T$ - операция транспонирования), отвечающий ее нулевому собственному значению, и предположим, что

$$
\left.\left(f_{y}^{\prime}(x, y), e_{0}\right)\right|_{x=\varphi_{1}\left(y_{3}\right), y=y_{3}} \neq 0
$$

где $(\cdot, \cdot)$ - евклидово скалярное произведение.

Перейдем теперь к матрице $A_{2}(y)$. Считаем, что, во-первых, при всех $y$ из интервала $y_{1}<y<y_{3}$ она имеет хотя бы одно собственное значение в правой комплексной полуплоскости $\{\lambda \in \mathbb{C}: \operatorname{Re} \lambda>0\}$ и, во-вторых, при $y=y_{1}$ одно ее собственное значение нулевое, другое отрицательно и выполняется аналогичное (1.15) неравенство.

Обратимся, далее, к матрице $A_{3}(y)$ и предположим, что существует значение $y=y_{2} \in\left(y_{1}, y_{3}\right)$ со следующими свойствами: при $y \in\left(y_{1}, y_{2}\right)$ эта матрица гурвицева, при $y \in\left(y_{2}, y_{3}\right)$ у нее имеется хотя бы одно собственное значение в правой комплексной полуплоскости, а при $y=y_{2}$ ее собственные значения чисто мнимые, т. е. $\lambda= \pm i \beta_{0}$, где $\beta_{0}>0$.

Очередные два ограничения относятся к системе (1.14) при значениях $y$, близких к $y_{2}$. В силу сказанного чуть выше при таких $y$ матрица $A_{3}(y)$ допускает комплексные собственные значения $\lambda=\alpha(y) \pm i \beta(y), \alpha(y), \beta(y) \in \mathbb{R}$, $\alpha\left(y_{2}\right)=0, \beta\left(y_{2}\right)=\beta_{0}$. Что же касается системы (1.14) при $y=y_{2}$, то в окрестности состояния равновесия $x=\varphi_{3}\left(y_{2}\right)$ ее нормальная форма Пуанкаре-Дюлака, выписанная с точностью до кубических слагаемых, имеет вид

$$
\frac{d z}{d \tau}=i \beta_{0} z+d|z|^{2} z, \quad d=\text { const } \in \mathbb{C},
$$


где $z$ - некоторая вспомогательная комплексная переменная. Будем считать, что

$$
\left.\alpha^{\prime}(y)\right|_{y=y_{2}}>0, \quad \operatorname{Re} d<0 .
$$

Как известно (см., например, [25]), при условиях (1.16) и при прохождении параметра $y$ через критическое значение $y=y_{2}$ в системе (1.14) происходит стандартная бифуркация Андронова-Хопфа, приводящая к появлению у нее при всех $0<y-y_{2} \ll 1$ экспоненциально орбитально устойчивого цикла

$$
\begin{gathered}
x=x_{*}(\theta, y): x_{*}\left(\theta, y_{2}\right) \equiv \varphi_{3}\left(y_{2}\right), \quad x_{*}(\theta+2 \pi, y) \equiv x_{*}(\theta, y), \\
\frac{d \theta}{d \tau}=\omega_{*}(y),
\end{gathered}
$$

где частота $\omega_{*}(y)>0$ такова, что $\omega_{*}\left(y_{2}\right)=\beta_{0}$. Будем считать, что этот цикл непрерывно продолжается по параметру $y$ (с сохранением экспоненциальной орбитальной устойчивости) на промежуток $y \in\left(y_{2}, y_{3}\right]$. Тогда равенство (1.17) задает в $\mathbb{R}^{3}$ гладкую поверхность

$$
\Gamma=\left\{(x, y) \in \mathbb{R}^{3}: x=x_{*}(\theta, y), 0 \leqslant \theta \leqslant 2 \pi, y_{2} \leqslant y \leqslant y_{3}\right\},
$$

вид которой показан на рис. 4 .

Последняя серия ограничений, связанных с системой (1.14), относится к ее фазовым портретам при $y=y_{1}$ и $y=y_{3}$. Обратимся сначала к случаю $y=y_{1}$ и заметим, что в малой окрестности критического состояния равновесия $x=\varphi_{3}\left(y_{1}\right)$ система $d x / d \tau=f\left(x, y_{1}\right)$ имеет экспоненциально устойчивое одномерное инвариантное центральное многообразие. Движения на этом многообразии описываются с помощью некоторого скалярного уравнения вида $\dot{r}=\Delta(r), \Delta(0)=\Delta^{\prime}(0)=0, r \in \mathbb{R}$. Считаем, что, во-первых, выполняется неравенство

$$
\Delta^{\prime \prime}(0) \neq 0
$$

и, во-вторых, единственная траектория $L_{1}$, лежащая на упомянутом многообразии и стремящаяся при $\tau \rightarrow-\infty$ к состоянию равновесия $x=\varphi_{3}\left(y_{1}\right)$, при $\tau \rightarrow+\infty$ стремится к экспоненциально устойчивому состоянию равновесия $x=\varphi_{1}\left(y_{1}\right)$ (см. рис. 4 , где соответствующий фазовый портрет изображен в плоскости $\left.y=y_{1}\right)$.

В случае $y=y_{3}$ для критического состояния равновесия $x=\varphi_{1}\left(y_{3}\right)$ системы $d x / d \tau=f\left(x, y_{3}\right)$ предполагаем выполненным аналогичное (1.19) неравенство. Считаем также, что единственная траектория $L_{2}$ этой системы, выходящая из точки $x=\varphi_{1}\left(y_{3}\right)$ (т. е. стремящаяся к $\varphi_{1}\left(y_{3}\right)$ при $\tau \rightarrow-\infty$ ), при $\tau \rightarrow+\infty$ стремится к устойчивому циклу (1.17) при $y=y_{3}$ (см. рис. 4).

Заключительные ограничения связаны с правой частью $g(x, y)$ из (1.13), а точнее говоря, с ее знаками. Будем считать, что

$$
\begin{gathered}
g\left(\varphi_{1}(y), y\right)>0 \quad \text { при } y_{1} \leqslant y \leqslant y_{3}, \quad g\left(\varphi_{3}(y), y\right)<0 \quad \text { при } y_{1} \leqslant y \leqslant y_{2}, \\
\widetilde{g}(y) \stackrel{\text { def }}{=} \frac{1}{2 \pi} \int_{0}^{2 \pi} g\left(x_{*}(\theta, y), y\right) d \theta<0 \quad \text { при } y_{2} \leqslant y \leqslant y_{3} .
\end{gathered}
$$


При сформулированных условиях, опираясь на результаты монографии [26], дадим качественное описание асимптотического поведения при $\varepsilon \rightarrow 0$ произвольной траектории

$$
(x(t, \varepsilon), y(t, \varepsilon)): \quad t \geqslant 0, \quad x(0, \varepsilon)=x_{0}, \quad y(0, \varepsilon)=y_{0}
$$

системы (1.13) с не зависящими от $\varepsilon$ начальными условиями $x_{0}, y_{0}$. Для определенности будем считать, что $y_{0} \in\left(y_{1}, y_{3}\right)$, а точка $x=x_{0}$ лежит в области притяжения устойчивого состояния равновесия $x=\varphi_{1}\left(y_{0}\right)$ системы $(1.14)$ при $y=y_{0}$.

Сначала происходит асимптотически быстрое (занимающее отрезок времени порядка $\varepsilon \ln (1 / \varepsilon))$ "падение" фазовой точки $(x(t, \varepsilon), y(t, \varepsilon))$ на устойчивую кривую медленных движений $x=\varphi_{1}(y)$. При этом переменная $x$ меняется примерно по закону $x=x\left(\tau, x_{0}\right), \tau=t / \varepsilon$, где $x\left(\tau, x_{0}\right)$ - решение задачи Коши $d x / d \tau=f\left(x, y_{0}\right),\left.x\right|_{\tau=0}=x_{0}$, а переменная $y$ асимптотически близка к константе $y=y_{0}$.

После падения начинается так называемый участок медленного движения. На этом участке фазовая точка $(x(t, \varepsilon), y(t, \varepsilon))$ асимптотически близка к кривой $\left\{(x, y): x=\varphi_{1}(y)\right\}$, а переменная $y$ с точностью до асимптотически малых добавок удовлетворяет задаче Коши

$$
\dot{y}=g\left(\varphi_{1}(y), y\right),\left.\quad y\right|_{t=0}=y_{0} .
$$

Далее, учитывая в (1.22) первое неравенство из (1.20), приходим к выводу, что за время, асимптотически близкое к значению

$$
T_{1}=\int_{y_{0}}^{y_{3}} \frac{d y}{g\left(\varphi_{1}(y), y\right)}>0,
$$

фазовая точка (1.21) попадает в асимптотически малую окрестность точки срыва $O_{2}=\left\{(x, y): x=\varphi_{1}\left(y_{3}\right), y=y_{3}\right\}$ (см. рис. 4$)$.

Последующий участок в теории релаксационных колебаний принято называть участком быстрого движения. Для того чтобы разобраться с ним, объединим локальные условия в точке $O_{2}$ (неравенства вида $(1.15),(1.19)$ ), обеспечивающие "срыв" с многообразия медленных движений $x=\varphi_{1}(y)$, с нелокальным поведением кривой $L_{2}$ (см. рис. 4). Согласно результатам монографии [26] на данном участке компонента $x(t, \varepsilon)$ меняется в асимптотически малой окрестности траектории $L_{2}$ и асимптотически быстро оказывается в асимптотически малой окрестности цикла (1.17) при $y=y_{3}$. Что же касается компоненты $y(t, \varepsilon)$, то она все это время остается асимптотически близкой к константе $y=y_{3}$.

После прохождения участка быстрого движения фазовая точка (1.21) начинает двигаться в асимптотически малой окрестности поверхности Г (см. рис. 4). При этом компонента $y(t, \varepsilon)$ убывает со скоростью порядка единицы, а компонента $x(t, \varepsilon)$ быстро осциллирует, повторяя форму колебаний циклов (1.17) при различных $y$. Точнее говоря, в первом приближении эволюция данных компонент задается соотношениями

$$
x=x_{*}(\theta, y), \quad \dot{\theta}=\frac{\omega_{*}(y)}{\varepsilon}, \quad \dot{y}=\widetilde{g}(y),\left.\quad y\right|_{t=T_{1}}=y_{3},
$$

где $T_{1}$ - величина (1.23). 
В связи с отмеченным выше характером поведения $x(t, \varepsilon)$ участок траектории (1.21), описываемый равенствами (1.24), будем называть bursting-участком. Учитывая в (1.24) третье условие (1.20), приходим к выводу, что этот участок занимает промежуток времени $T_{1} \lesssim t \lesssim T_{2}$, где

$$
T_{2}=T_{1}-\int_{y_{2}}^{y_{3}} \frac{d y}{\widetilde{g}(y)}>T_{1} .
$$

При прохождении же $t$ через значение (1.25) осцилляция компоненты $x(t, \varepsilon)$ прекращается и bursting-участок плавно переходит в очередной участок медленных движений, описываемый (в первом приближении) аналогичными (1.22) формулами

$$
x=\varphi_{3}(y), \quad \dot{y}=g\left(\varphi_{3}(y), y\right),\left.\quad y\right|_{t=T_{2}}=y_{2} .
$$

Из второго неравенства (1.20) вытекает, что соотношения (1.26) справедливы до тех пор, пока фазовая точка (1.21) не попадет в асимптотически малую окрестность точки срыва $O_{1}=\left\{(x, y): x=\varphi_{3}\left(y_{1}\right), y=y_{1}\right\}$ (см. рис. 4). Последнее же происходит при значениях $t$, асимптотически близких к $t=T_{3}$, где

$$
T_{3}=T_{2}-\int_{y_{1}}^{y_{2}} \frac{d y}{g\left(\varphi_{3}(y), y\right)}>T_{2} .
$$

Затем следует очередной участок быстрых движений, аналогичный уже описанному выше. На этом участке компонента $y(t, \varepsilon)$ асимптотически близка к значению $y=y_{1}$, а компонента $x(t, \varepsilon)$, двигаясь в асимптотически малой окрестности кривой $L_{1}$ (см. рис. 4$)$, асимптотически быстро оказывается в асимптотически малой окрестности точки $x=\varphi_{1}\left(y_{1}\right)$.

На заключительном этапе снова имеем дело с участком медленных движений, задающимся аналогичными (1.22) равенствами

$$
x=\varphi_{1}(y), \quad \dot{y}=g\left(\varphi_{1}(y), y\right),\left.\quad y\right|_{t=T_{3}}=y_{1},
$$

где $T_{3}$ - момент времени (1.27). Из соотношений $(1.20),(1.28)$, в свою очередь, следует существование момента времени $t=T_{*}(\varepsilon)$ (являющегося вторым положительным корнем уравнения $\left.y(t, \varepsilon)=y_{0}\right)$, при котором фазовая точка (1.21) возвращается в начальную плоскость $y=y_{0}$ и попадает в асимптотически малую окрестность точки $(x, y)=\left(\varphi_{1}\left(y_{0}\right), y_{0}\right)$. Нетрудно проверить, что $T_{*}(0)=T_{4}$, где

$$
T_{4}=T_{3}+\int_{y_{1}}^{y_{0}} \frac{d y}{g\left(\varphi_{1}(y), y\right)}>T_{3} .
$$

Суммируя вышесказанное, приходим к выводу, что в некотором замкнутом шаре $B \subset \mathbb{R}^{2}$ с центром в точке $x=\varphi_{1}\left(y_{0}\right)$, целиком лежащем в области притяжения состояния равновесия $\varphi_{1}\left(y_{0}\right)$ системы $(1.14)$ при $y=y_{0}$, определен оператор последования П: $\left.x_{0} \in B \rightarrow x(t, \varepsilon)\right|_{t=T_{*}(\varepsilon)}$ по траекториям (1.21) системы (1.13). Содержащиеся в [26] результаты позволяют утверждать, что оператор П преобразует шар $B$ в себя и является в нем сжимающим. Тем самым, он допускает единственную неподвижную точку, которой в системе (1.13) соответствует устойчивый релаксационный цикл. Данный цикл уместно называть bursting-циклом в силу характера зависимости от $t$ его компоненты 
$x=\left(x_{1}, x_{2}\right)$. Действительно, с течением времени участки быстрой осцилляции функций $x_{1}, x_{2}$ чередуются с участками медленных движений, т. е. имеет место некая аналогия с bursting-активностью биологического нейрона.

Подчеркнем, что нами предложен простейший набор условий, гарантирующих существование у системы (1.13) устойчивых автоколебаний с bursting-эффектом. Но возможны и другие варианты таких условий. В связи с этим сформулируем три общих принципа существования устойчивых bursting-колебаний.

Во-первых, должен быть некоторый промежуток изменения $y$, в котором у системы (1.14) сосуществуют устойчивое состояние равновесия $x=\varphi(y)$ и устойчивый цикл $x=x_{*}(\theta, y), x_{*}(\theta+2 \pi, y) \equiv x_{*}(\theta, y), d \theta / d \tau=\omega_{*}(y), \omega_{*}(y)>0$. Это так называемое условие бистабильности.

Во-вторых, на упомянутом промежутке должно выполняться неравенство $g(\varphi(y), y) \cdot \widetilde{g}(y)<0$, где $\widetilde{g}(y)-$ функция из (1.20). Данное условие, которое уместно назвать условием гистерезиса, обеспечивает разнонаправленность движений вдоль медленной кривой $S=\{(x, y): x=\varphi(y)\}$ и вдоль поверхности $\Gamma=\left\{(x, y): x=x_{*}(\theta, y), 0 \leqslant \theta \leqslant 2 \pi\right\}$.

В-третьих, должны существовать механизмы переключений, позволяющие траекториям системы (1.13) переходить с кривой $S$ на поверхность Г и обратно. Эти механизмы зависят от происходящих в системе (1.14) бифуркаций и могут быть разными. Простейшие из них описаны нами выше.

Остановимся на некоторых конкретных примерах систем вида (1.13), возникающих в нейродинамике. Одной из них является так называемая модель Фитцхью-Ринцеля, имеющая вид

$$
\varepsilon \dot{x}_{1}=x_{1}-\frac{x_{1}^{3}}{3}-x_{2}+y+I, \quad \varepsilon \dot{x}_{2}=\delta\left(a+x_{1}-b x_{2}\right), \quad \dot{y}=c-x_{1}-d y .
$$

В работе [21] с помощью численных методов было установлено существование у системы (1.29) устойчивого bursting-цикла при $I=0.3125, a=0.7, b=0.8$, $c=-0.775, d=1, \delta=0.08, \varepsilon \leqslant 0.0001$.

Другим представителем систем (1.13) служит модель Хиндмарша и Роуз [15]

$$
\varepsilon \dot{x}_{1}=x_{2}-a x_{1}^{3}+b x_{1}^{2}+I-y, \quad \varepsilon \dot{x}_{2}=c-d x_{1}^{2}-x_{2}, \quad \dot{y}=k_{1}\left(x_{1}-k_{2}\right)-y .
$$

Чаще всего систему (1.30) рассматривают при значениях параметров $a=1$, $b=3, c=1, d=5, k_{1}=4, k_{2}=-8 / 5,|I| \leqslant 10, \varepsilon \leqslant 0.001$. Как показал численный анализ (см. [15]), в этом случае она имеет устойчивые автоколебательные режимы с bursting-участками.

В дополнение к приведенным примерам сошлемся на статью [18], где представлена целая серия трехмерных моделей с bursting-эффектом. Некоторые из них получаются путем достройки модели (1.10), (1.11) до трехмерной системы. Например, если положить в (1.10), (1.11)

$$
I=I(u), \quad I(u)=-u, \quad \dot{u}=\varepsilon(0.2+V), \quad 0<\varepsilon \ll 1,
$$


где $u$ - новая переменная, и выполнить нормировку $\varepsilon t \rightarrow t$, то после соответствующих переобозначений получим систему вида (1.13). Как показал численный эксперимент (см. [18]), при значениях параметров

$$
\begin{gathered}
C=1, \quad \lambda=1 / 3, \quad V_{1}=-0.01, \quad V_{2}=0.15, \quad V_{3}=0.1, \quad V_{4}=0.05, \quad V_{l}=-0.5, \\
V_{\mathrm{K}}=-0.7, \quad V_{\mathrm{Ca}}=1, \quad \bar{g}_{l}=0.5, \quad \bar{g}_{\mathrm{K}}=2, \quad \bar{g}_{\mathrm{Ca}}=1.2
\end{gathered}
$$

и при $\varepsilon \leqslant 0.005$ система (1.10), (1.11), (1.31) имеет устойчивый bursting-цикл.

Следует отметить, что для всех приведенных здесь примеров выполняются три сформулированных выше общих принципа существования устойчивых bursting-автоколебаний: бистабильность, гистерезис и наличие требуемых механизмов переключений. Однако сами эти механизмы отличны от описанного нами простейшего варианта.

1.4. Модель Хопфилда с запаздыванием. Перейдем теперь к нейронным моделям с запаздыванием. Поскольку об этих моделях еще будет идти речь в п. 1.6, то здесь мы ограничимся лишь одним наиболее известным примером - системой Хопфилда с запаздыванием. История возникновения упомянутой системы такова.

В 1984 г. Хопфилд [27] предложил математическую модель простейшей нейронной сети, представляющую собой систему обыкновенных дифференциальных уравнений вида

$$
\dot{u}_{j}=-\mu_{j} u_{j}+\sum_{i=1}^{m} a_{i j} f_{i}\left(u_{i}\right)+I_{j}, \quad j=1, \ldots, m .
$$

Здесь $u_{j}(t)$ - мембранные потенциалы нейронов, $\mu_{j}=$ const $\geqslant 0$ - коэффициенты затухания за счет токов утечки, $a_{i j}=$ const $\in \mathbb{R}$ - синаптические веса, $I_{j}=$ const $\in \mathbb{R}$ - внешние токи смещения, а гладкие функции $f_{j}(u), u \in \mathbb{R}$, представляющие собой вольт-амперные характеристики нелинейных элементов, удовлетворяют предельным равенствам

$$
\lim _{u \rightarrow-\infty} f_{j}(u)=0, \quad \lim _{u \rightarrow+\infty} f_{j}(u)=1 .
$$

Достаточно подробное описание свойств этой системы, а также ее вывод можно найти в монографии [28].

Автор работы [27] исходил из допущения, что все элементы сети оказывают друг на друга мгновенное воздействие. Однако в реальности взаимодействие нейронов происходит с некоторыми запаздываниями по времени, обусловленными, например, конечной скоростью переключения усилителей в электрической нейронной сети или конечной скоростью распространения сигнала в биологических сетях. Таким образом, представляется вполне осмысленным переход от системы (1.32) к системе дифференциально-разностных уравнений

$$
\dot{u}_{j}=-\mu_{j} u_{j}+\sum_{i=1}^{m} a_{i j} f_{i}\left(u_{i}\left(t-s_{i j}\right)\right)+I_{j}, \quad j=1, \ldots, m,
$$

где $s_{i j}=$ const $\geqslant 0$. Впервые такой переход был осуществлен в статье [29], а в последующем как сама система (1.34), так и некоторые ее модификации 
изучались многими авторами (см., например, работы [30]-[36] и содержащуюся в них библиографию). Как правило, во всех этих статьях рассматривались вопросы об устойчивости состояния равновесия и о возникновении незатухающих автоколебаний в результате бифуркации Андронова-Хопфа.

Отдельно остановимся на статье [37], в которой в отличие от упомянутых выше работ исследовались не гармонические, а релаксационные автоколебания в сетях Хопфилда. Точнее говоря, в [37] изучалась кольцевая система

$$
\begin{gathered}
\dot{u}_{j}=-\mu u_{j}+\lambda\left[1-(a+1) f\left(u_{j}(t-1)\right)-b g\left(u_{j-1}\right)\right], \\
j=1, \ldots, m, \quad u_{0}=u_{m},
\end{gathered}
$$

где $\mu, a, b=$ const $>0$, функции $f(u), g(u)$ обладали свойствами (1.33), а параметр $\lambda>0$ предполагался большим. Было установлено, что при подходящем выборе параметров $\mu, a, b$ и при согласованном увеличении $\lambda, m$ можно добиться сосуществования в системе (1.35) любого наперед заданного конечного числа устойчивых релаксационных циклов. Иными словами, в ней реализуется известный феномен буферности (см. [38], [39]).

1.5. Основные способы моделирования нейронных сетей. Обратим внимание на то, что за исключением систем (1.32), (1.34), (1.35) нами рассматривались только модели изолированного нейрона. Это вполне естественно, так как создание таких моделей - первый этап в решении более общей проблемы: математического моделирования нейронных ассоциаций и сетей. Вторым же этапом указанной проблемы является математическое описание межнейронных связей - как электрических, так и химических.

В случае электрических связей способ действий таков. Пусть функционирование отдельного нейрона моделируется, к примеру, системой (1.8). Тогда одна из возможных математических моделей цепочки взаимодействующих нейронов имеет вид

$$
\varepsilon \dot{u}_{j}=d\left(u_{j+1}-2 u_{j}+u_{j-1}\right)+f\left(u_{j}, v_{j}\right), \quad \dot{v}_{j}=g\left(u_{j}, v_{j}\right), \quad j=1, \ldots, m,
$$

где $u_{0}=u_{1}, u_{m+1}=u_{m}$, а параметр $d \in \mathbb{R}$ характеризует глубину связи между нейронами.

Существуют и другие способы описания электрического взаимодействия между нейронами. Например, вместо системы (1.36) можно рассмотреть кольцевую цепочку вида

$$
\varepsilon \dot{u}_{j}=d\left(u_{j+1}-u_{j}\right)+f\left(u_{j}, v_{j}\right), \quad \dot{v}_{j}=g\left(u_{j}, v_{j}\right), \quad j=1, \ldots, m,
$$

где $u_{m+1}=u_{1}, d \in \mathbb{R}$, или полносвязную нейронную сеть

$$
\varepsilon \dot{u}_{j}=\sum_{\substack{i=1 \\ i \neq j}}^{m} d_{i j}\left(u_{i}-u_{j}\right)+f\left(u_{j}, v_{j}\right), \quad \dot{v}_{j}=g\left(u_{j}, v_{j}\right), \quad j=1, \ldots, m,
$$

где $d_{i j}=$ const $\in \mathbb{R}$. Можно также аналогичные линейные слагаемые, отвечающие за электрическое взаимодействие нейронов, добавлять и в уравнения для переменных $v_{j}, j=1, \ldots, m$. 
Другой способ взаимодействия нервных клеток - синаптический. Системы с ингибиторной связью (химический синапс) рассмотрены в большом числе статей (см., например, [40]-[42]). В основе подхода к моделированию химических синапсов лежит идея быстрой пороговой модуляции. Феномен быстрой пороговой модуляции (fast threshold modulation, или FTM), впервые описанный в работах [43], [44], представляет собой специальный способ связи динамических систем. Характерная особенность этого способа состоит в том, что правые части соответствующих дифференциальных уравнений меняются скачкообразно при переходе некоторых управляющих переменных через свои критические значения. В нейронных системах идея FTM реализуется, как правило, следующим образом.

Предположим, что напряжение $u=u(t)$ и сила тока $v=v(t)$ в отдельной нейронной клетке, как и выше, удовлетворяют системе (1.8). Рассмотрим, далее, простейшую сеть, состоящую из двух синаптически связанных нейронов. В этом случае согласно выработанным к настоящему времени представлениям (см., например, [45]) соответствующие им электрические переменные $\left(u_{j}, v_{j}\right)$, $j=1,2$, удовлетворяют системе уравнений

$$
\begin{array}{ll}
\varepsilon \dot{u}_{1}=f\left(u_{1}, v_{1}\right)+b s_{2}\left(u_{2}\right)\left(u_{*}-u_{1}\right), & \dot{v}_{1}=g\left(u_{1}, v_{1}\right), \\
\varepsilon \dot{u}_{2}=f\left(u_{2}, v_{2}\right)+b s_{1}\left(u_{1}\right)\left(u_{*}-u_{2}\right), & \dot{v}_{2}=g\left(u_{2}, v_{2}\right) .
\end{array}
$$

Здесь $b$ - положительный параметр, характеризующий максимальную проводимость синапса, $u_{*}$ - потенциал покоя (или потенциал Нернста), а функции $s_{j}\left(u_{j}\right), j=1,2,-$ постсинаптические проводимости, зависящие от пресинаптических потенциалов $u_{j}$.

Следует отметить, что существует несколько различных способов выбора функций $s_{j}\left(u_{j}\right)$, описание которых можно найти в [45]. Мы же, руководствуясь идеей FTM, остановимся здесь лишь на простейшем из них. А именно, будем считать, что

$$
s_{j}\left(u_{j}\right)=H\left(u_{j}-u_{* *}\right), \quad H(x)= \begin{cases}0 & \text { при } x<0, \\ 1 & \text { при } x>0,\end{cases}
$$

где $u_{* *}-$ порог, начиная с которого одна клетка влияет на другую. Например, если $u_{1}<u_{* *}$, то первый нейрон не действует на второй, а если $u_{1}>u_{* *}$, то действует.

В заключение добавим, что между нейронами могут существовать одновременно как электрические, так и химические связи. Приведенные выше общие принципы позволяют получить соответствующие математические модели в этом случае.

1.6. Новый подход к моделированию отдельного нейрона. В данном пункте приведем описание нашей собственной модели функционирования отдельного нейрона, в которой учтены эффекты запаздывания.

Опираясь на подход Ходжкина и Хаксли (см. [4], [5]), заменим биологический нейрон электросхемой, показанной на рис. 1, но теперь будем считать положительными токи, направленные изнутри клетки во внешнюю среду. Предположим, далее, что суммарный мембранный ток $I$ и ток утечки $I_{l}$ равны нулю, 
а в качестве начала отсчета мембранного потенциала $E(t)$ возьмем $E_{*}$, где $E_{*}-$ уровень наибольшей поляризации мембраны (который, напомним, отрицателен). В этом случае $E(t)=E_{*}+u(t), u(t)>0$, а уравнение баланса токов из (1.5) примет вид

$$
C \dot{u}=-I_{\mathrm{Na}}-I_{\mathrm{K}},
$$

где, как и ранее, $C=$ const $>0$.

Для формирования содержательной модели сделаем несколько дополнительных предположений.

Условие 1.1. Считаем, что токи $I_{\mathrm{Na}}$ и $I_{\mathrm{K}}$ записываются в виде

$$
I_{\mathrm{Na}}=\chi_{\mathrm{Na}}\left(u\left(t-h_{1}\right)\right) \cdot u, \quad I_{\mathrm{K}}=\chi_{\mathrm{K}}\left(u\left(t-h_{2}\right)\right) \cdot u .
$$

Здесь непрерывные по $u \in \mathbb{R}_{+}, \mathbb{R}_{+}=\{u \in \mathbb{R}: u \geqslant 0\}$, бункиии $\chi_{\mathrm{Na}}(u), \chi_{\mathrm{K}}(u)$ представляют собой натриевую и калиевую проводимости, $а h_{1}, h_{2}>0$ - запаздывания по времени.

УСловие 1.2. Предполагаем, что $\chi_{\mathrm{Na}}(0)=\alpha_{0} u \chi_{\mathrm{Na}}(u) \rightarrow \beta_{0}$ при $u \rightarrow+\infty$, причем $\beta_{0}>\alpha_{0}>0$.

Положительность величины $\chi_{\mathrm{Na}}(0)$ легко объяснить, исходя из описанных в п. 1.1 биофизических механизмов функционирования отдельного нейрона. Действительно, в состоянии гиперполяризации $(u \ll 1)$ на внутренней поверхности мембраны наблюдается избыток ионов натрия. В их откачивании из клетки и состоит работа ионных насосов. В силу выбранной нами ориентации токов в этом случае ток $I_{\mathrm{Na}}$ заведомо положителен. А отсюда и из (1.42) заключаем, что $\chi_{\mathrm{Na}}(u)>0$ при всех $u \ll 1$.

Перейдем к обсуждению зависимости от $u$ функции $\chi_{\mathrm{K}}(u)$. В связи с этим обратим внимание на то, что в состоянии гиперполяризации поток ионов калия направлен внутрь клетки, а значит, $I_{\mathrm{K}}<0$ и автоматически $\chi_{\mathrm{K}}(u)<0$ при $u \ll 1$. Однако во время прохождения спайка, т. е. при $u \gg 1$, данный поток меняет свое направление. Следовательно, существует такое пороговое значение переменной $u$, при превышении которого проводимость $\chi_{\mathrm{K}}(u)$ становится положительной. Учитывая вышесказанное, приходим к следующему условию.

УСловие 1.3. Сиитаем, что $\chi_{\mathrm{K}}(0)=-\alpha_{1} u \chi_{\mathrm{K}}(u) \rightarrow \beta_{1}$ при $u \rightarrow+\infty$, где $\alpha_{1}, \beta_{1}>0$.

Соотношения (1.41), (1.42) приводят к выводу, что в рамках сделанных нами допущений эволюция во времени мембранного потенциала $u=u(t)$ описывается дифференциально-разностным уравнением

$$
C \dot{u}=-\left[\chi_{\mathrm{Na}}\left(u\left(t-h_{1}\right)\right)+\chi_{\mathrm{K}}\left(u\left(t-h_{2}\right)\right)\right] u .
$$

Это уравнение имеет, очевидно, нулевое состояния равновесия, которое в случае импульсного нейрона заведомо неустойчиво. Тем самым, должно выполняться неравенство

$$
-\chi_{\mathrm{Na}}(0)-\chi_{\mathrm{K}}(0)=\alpha_{1}-\alpha_{0}>0 .
$$

Мы же будем считать, что справедливо следующее, более сильное, чем (1.44), ограничение. 
УСловие 1.4. Предполагаем, что

$$
\alpha_{1} \in\left(\alpha_{0}, \beta_{0}\right) .
$$

Для удобства дальнейшего анализа преобразуем уравнение (1.43) к некоторой канонической форме. С этой целью введем в рассмотрение функции

$$
f(u)=\frac{\chi_{\mathrm{Na}}(u)+\chi_{\mathrm{K}}(0)}{\chi_{\mathrm{Na}}(0)+\chi_{\mathrm{K}}(0)}, \quad g(u)=-\frac{\chi_{\mathrm{K}}(u)-\chi_{\mathrm{K}}(0)}{\chi_{\mathrm{Na}}(0)+\chi_{\mathrm{K}}(0)},
$$

с помощью которых наше уравнение записывается в виде

$$
\dot{u}=r\left[f\left(u\left(t-h_{1}\right)\right)-g\left(u\left(t-h_{2}\right)\right)\right] u,
$$

где $r=-\left(\chi_{\mathrm{Na}}(0)+\chi_{\mathrm{K}}(0)\right) / C>0$ (см. (1.44)). Выполняя затем в (1.47) замену времени $t / h_{2} \rightarrow t$, окончательно получаем:

$$
\dot{u}=\lambda[f(u(t-h))-g(u(t-1))] u,
$$

где $\lambda=r h_{2}, h=h_{1} / h_{2}$.

Уравнение (1.48) и является интересующей нас математической моделью отдельного нейрона. Эта модель носит заведомо феноменологический характер, поскольку лежащие в ее основе условия 1.1-1.4 поддаются биофизическому объяснению лишь частично. Но тем не менее с ее помощью описываются такие фундаментальные нейродинамические эффекты, как генерация спайков, bursting-активность и феномен буферности.

Необходимо отметить, что наш подход к моделированию отдельного нейрона в идейном плане смыкается с подходом, предложенным в книге [46]. В упомянутой монографии при несколько иных гипотезах о натриевой и калиевой проводимостях получена аналогичная (1.48) вольтерровская модель с одним запаздыванием. Однако в случае одного запаздывания можно объяснить лишь факт генерации спайков. Добиться же реализуемости в такой модели bursting-эффекта и буферности не удается. Тем самым, становится понятной принципиальная важность учета второго запаздывания.

Математическое исследование модели (1.48) будем проводить при условии $\lambda \gg 1$ (в силу изложенных в п. 1.1 биофизических соображений это допущение вполне естественно) и при некоторых дополнительных ограничениях на функции (1.46). А именно, предположим, что $f, g \in C^{2}\left(\mathbb{R}_{+}\right)$. Далее, из условий 1.1-1.4 и формул (1.46) вытекает, что

$$
f(0)=1, \quad g(0)=0, \quad \lim _{u \rightarrow+\infty} f(u)=-a, \quad \lim _{u \rightarrow+\infty} g(u)=b,
$$

где постоянные $a, b$ задаются равенствами

$$
a=-\frac{\alpha_{1}-\beta_{0}}{\alpha_{1}-\alpha_{0}}, \quad b=\frac{\alpha_{1}+\beta_{1}}{\alpha_{1}-\alpha_{0}}
$$

и в силу (1.45) являются положительными. Мы же усилим требования (1.49) и будем считать, что

$$
\begin{array}{lll}
f(u)=-a+O\left(u^{-1}\right), & u f^{\prime}(u)=O\left(u^{-1}\right), & u^{2} f^{\prime \prime}(u)=O\left(u^{-1}\right), \\
g(u)=b+O\left(u^{-1}\right), & u g^{\prime}(u)=O\left(u^{-1}\right), & u^{2} g^{\prime \prime}(u)=O\left(u^{-1}\right)
\end{array}
$$

при $u \rightarrow+\infty$. 
Помимо уравнения (1.48) ниже рассматривается и его упрощенный аналог

$$
\dot{u}=\lambda f(u(t-1)) u,
$$

где функция $f(u)$ удовлетворяет всем перечисленным выше условиям. Уравнение (1.51) получается из (1.48) при $h=1$ и при переобозначениях

$$
f(u)-g(u) \rightarrow f(u), \quad a+b \rightarrow a .
$$

Кроме того, оно интересно еще и тем, что представляет собой некое обобщение известного уравнения Хатчинсона [47]

$$
\dot{u}=\lambda(1-u(t-1)) u
$$

из экологии. А именно, уравнение (1.52) получается из (1.51) при аппроксимации функции $f(u)$, меняющей знак c “+" на “-" при увеличении $u$, линейной функцией $f(u)=1-u$.

В настоящей статье излагаются специальные асимптотические методы, позволяющие разобраться с вопросами о существовании и устойчивости релаксационных периодических движений как в самих уравнениях (1.48), (1.51), так и в системах связанных уравнений такого типа. На этом пути устанавливается реализуемость в данных моделях bursting-эффекта и феномена буферности.

\section{2. Математическая модель импульсного нейрона с одним запаздыванием}

2.1. Основной результат. В настоящем разделе изучается модель (1.51). Как будет показано ниже, при выполнении условий (1.49), (1.50) на функцию $f(u)$ уравнение (1.51) при всех $\lambda \gg 1$ допускает устойчивый релаксационный цикл $u_{*}(t, \lambda), u_{*}(0, \lambda) \equiv 1$, периода $T_{*}(\lambda)$, обладающий свойствами

$$
\begin{gathered}
\lim _{\lambda \rightarrow+\infty} T_{*}(\lambda)=T_{0}, \quad T_{0}=2+a+\frac{1}{a}, \\
\max _{t} u_{*}(t, \lambda) \sim c_{1} \exp \lambda, \quad \min _{t} u_{*}(t, \lambda) \sim c_{2} \exp (-a \lambda),
\end{gathered}
$$

где $c_{1}, c_{2}=$ const $>0$. Наглядное представление об этом цикле дает показанный на рис. 5 график на плоскости $(t, u)$ функции $u=u_{*}(t+c, \lambda)$, где $c$ - некоторый фазовый сдвиг. Построение данного графика проводилось численно для случая $\lambda=7, f(u)=(1-u) /(1+0.5 u)$.

При исследовании вопроса о существовании у уравнения (1.51) релаксационного цикла $u_{*}(t, \lambda)$ с требуемыми характеристиками $(2.1),(2.2)$ и его устойчивости удобно сделать в (1.51) замену $u=\exp (\lambda x)$. Действительно, указанная замена преобразует интересующее нас уравнение к виду

$$
\dot{x}=F(x(t-1), \varepsilon),
$$

где $F(x, \varepsilon)=f(\exp (x / \varepsilon)), \varepsilon=1 / \lambda \ll 1$. Далее, свойства (1.49), (1.50) функции $f(u)$ позволяют заключить, что

$$
\lim _{\varepsilon \rightarrow 0} F(x, \varepsilon)=R(x), \quad R(x)=\left\{\begin{array}{rc}
1 & \text { при } x<0, \\
-a & \text { при } x>0 .
\end{array}\right.
$$




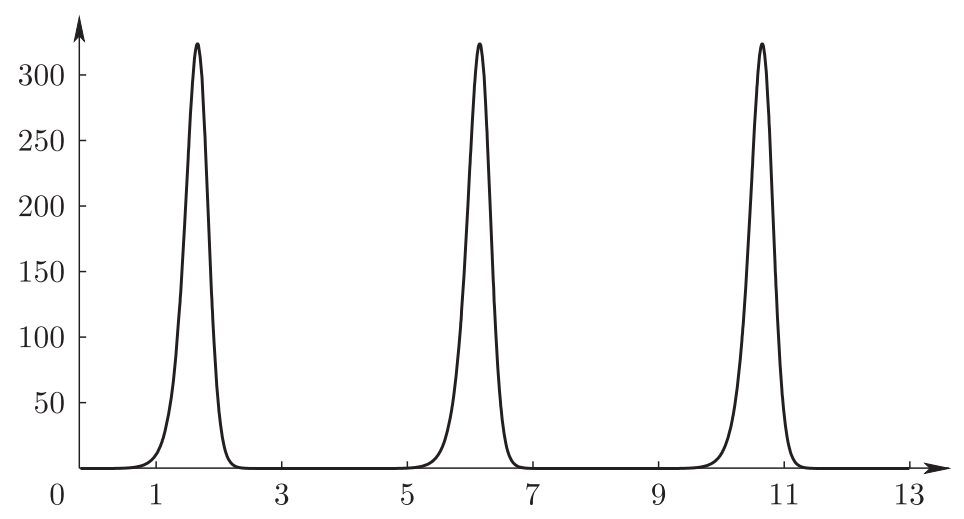

Рис. 5

Таким образом, исходному сингулярно возмущенному уравнению (1.51) ставится в соответствие предельное релейное уравнение с запаздыванием

$$
\dot{x}=R(x(t-1)) .
$$

Как и в работе [48], понятие решения уравнения (2.4) определим конструктивно. С этой целью зафиксируем некоторое

$$
\sigma_{0}: 0<\sigma_{0}<a,
$$

рассмотрим множество функций

$$
\varphi(t) \in C\left[-1-\sigma_{0},-\sigma_{0}\right], \quad \varphi(t)<0 \quad \forall t \in\left[-1-\sigma_{0},-\sigma_{0}\right], \quad \varphi\left(-\sigma_{0}\right)=-\sigma_{0},
$$

и обозначим через $x_{\varphi}(t), t \geqslant-\sigma_{0}$, решение уравнения $(2.4)$ с произвольной начальной функцией (2.6). Заметим, далее, что поскольку $\varphi(t-1)<0$ при $t \in\left[-\sigma_{0}, 1-\sigma_{0}\right]$, то на указанном промежутке времени согласно $(2.4) x_{\varphi}(t)$ является решением задачи Коши $\dot{x}=1, x\left(-\sigma_{0}\right)=-\sigma_{0}$ и, следовательно,

$$
x_{\varphi}(t)=t .
$$

Ясно также, что формула (2.7) сохраняется до тех пор, пока $x_{\varphi}(t-1)<0$. Тем самым, она справедлива на полуинтервале $-\sigma_{0} \leqslant t<1$.

При $t=1$ первый раз происходит переключение, и при $t>1$ решение $x_{\varphi}(t)$ определяется уже из задачи Коши $\dot{x}=-a, x(1)=1$, т. е. посредством равенства

$$
x_{\varphi}(t)=1-a(t-1) .
$$

В свою очередь, формула (2.8) остается в силе, пока $x_{\varphi}(t-1)>0$, т. е. до очередного момента переключения $t=t_{0}+1$, где

$$
t_{0}=1+\frac{1}{a}
$$

На промежутке же $t_{0}+1 \leqslant t \leqslant T_{0}-\sigma_{0}$, где $T_{0}$ определено в $(2.1)$, функция $x_{\varphi}(t)$ задается аналогичным (2.7) равенством

$$
x_{\varphi}(t)=-a+t-t_{0}-1 .
$$




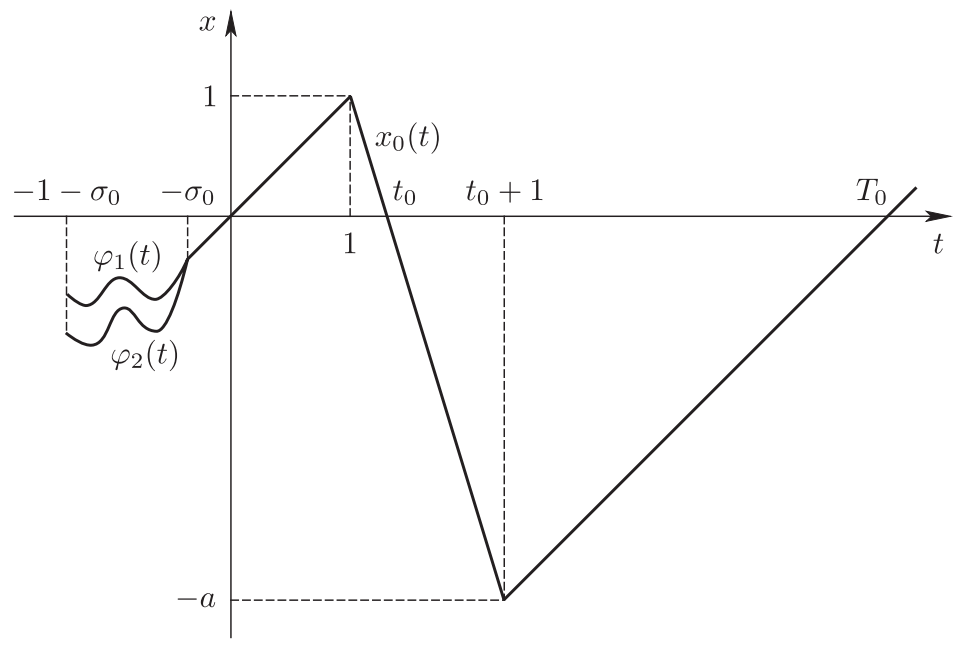

Рис. 6

Завершая описание построения решения $x_{\varphi}(t)$, отметим, что в силу условия (2.5) на постоянную $\sigma_{0}$ функция $x_{\varphi}\left(t+T_{0}\right),-1-\sigma_{0} \leqslant t \leqslant-\sigma_{0}$, принадлежит исходному начальному множеству (2.6). А это означает, что при $t \geqslant T_{0}-\sigma_{0}$ весь процесс повторяется. Более того, из (2.7)-(2.10) вытекает, что каждое решение $x_{\varphi}(t)$ с начальным условием $(2.6)$ при всех $t \geqslant-\sigma_{0}$ совпадает с одной и той же $T_{0}$-периодической функцией (см. рис. 6)

$$
x_{0}(t)=\left\{\begin{array}{ll}
t & \text { при } 0 \leqslant t \leqslant 1, \\
1-a(t-1) & \text { при } 1 \leqslant t \leqslant t_{0}+1, \\
-a+t-t_{0}-1 & \text { при } t_{0}+1 \leqslant t \leqslant T_{0},
\end{array} \quad x_{0}\left(t+T_{0}\right) \equiv x_{0}(t) .\right.
$$

Перейдем к вопросу о связи между периодическими решениями уравнений (2.3) и (2.4). Справедливо следующее утверждение.

Теорема 2.1. При всех достаточно малых $\varepsilon>0$ уравнение (2.3) допускает единственный экспоненииально орбитально устойчивый иикл $x=x_{*}(t, \varepsilon)$, $x_{*}\left(-\sigma_{0}, \varepsilon\right) \equiv-\sigma_{0}$, периода $T_{*}(\varepsilon)$, удовлетворяющий предельным равенствам

$$
\lim _{\varepsilon \rightarrow 0} \max _{0 \leqslant t \leqslant T_{*}(\varepsilon)}\left|x_{*}(t, \varepsilon)-x_{0}(t)\right|=0, \quad \lim _{\varepsilon \rightarrow 0} T_{*}(\varepsilon)=T_{0} .
$$

Доказательство сформулированной теоремы, содержащееся в двух следующих пунктах, опирается на некоторые дополнительные конструкции. Для их описания наряду с константой $\sigma_{0}$ (см. (2.5)) зафиксируем постоянные $q_{1}>\sigma_{0}$, $q_{2} \in\left(0, \sigma_{0}\right)$ и обозначим через $S\left(\sigma_{0}, q_{1}, q_{2}\right) \subset C\left[-1-\sigma_{0},-\sigma_{0}\right]$ замкнутое, ограниченное и выпуклое множество функций $\varphi(t)$, удовлетворяющих требованиям

$$
-q_{1} \leqslant \varphi(t) \leqslant-q_{2}, \quad \varphi\left(-\sigma_{0}\right)=-\sigma_{0} .
$$

Далее, для произвольной функции $\varphi \in S\left(\sigma_{0}, q_{1}, q_{2}\right)$ введем в рассмотрение решение $x=x_{\varphi}(t, \varepsilon), t \geqslant-\sigma_{0}$, уравнения $(2.3)$ с начальным условием $\varphi(t)$, 
$-1-\sigma_{0} \leqslant t \leqslant-\sigma_{0}$, а через $t=T_{\varphi}$ обозначим второй положительный корень уравнения $x_{\varphi}\left(t-\sigma_{0}, \varepsilon\right)=-\sigma_{0}$ (если он существует). И наконец, зададим оператор $\Pi_{\varepsilon}: S\left(\sigma_{0}, q_{1}, q_{2}\right) \rightarrow C\left[-1-\sigma_{0},-\sigma_{0}\right]$ с помощью равенства

$$
\Pi_{\varepsilon}(\varphi)=x_{\varphi}\left(t+T_{\varphi}, \varepsilon\right), \quad-1-\sigma_{0} \leqslant t \leqslant-\sigma_{0} .
$$

Последующий план действий таков. Сначала мы установим асимптотические формулы для $x_{\varphi}(t, \varepsilon)$ на различных промежутках изменения $t$, из которых будет следовать, что при подходящем выборе параметров $\sigma_{0}, q_{1}, q_{2}$ оператор (2.14) определен на множестве $S\left(\sigma_{0}, q_{1}, q_{2}\right)$ и преобразует его в себя. Затем проведем анализ уравнения в вариациях на решении $x_{\varphi}(t, \varepsilon)$ и покажем, что $\Pi_{\varepsilon}$ является сжимающим.

2.2. Существование релаксационного цикла. Построение асимптотики функции $x_{\varphi}(t, \varepsilon)$ начнем с отрезка $-\sigma_{0} \leqslant t \leqslant 1-\sigma_{0}$, на котором согласно $(2.3)$ эта функция является решением задачи Коши

$$
\dot{x}=F(\varphi(t-1), \varepsilon),\left.\quad x\right|_{t=-\sigma_{0}}=-\sigma_{0} .
$$

Далее, опираясь на неравенства из (2.13) и свойства (1.49), (1.50), замечаем, что равномерно по $t \in\left[-\sigma_{0}, 1-\sigma_{0}\right], \varphi \in S\left(\sigma_{0}, q_{1}, q_{2}\right)$

$$
F(\varphi(t-1), \varepsilon)=1+O\left(\exp \left(-\frac{q_{2}}{\varepsilon}\right)\right), \quad \varepsilon \rightarrow 0 .
$$

А отсюда очевидным образом выводим, что

$$
x_{\varphi}(t, \varepsilon)=t+\Delta_{1, \varphi}(t, \varepsilon), \quad-\sigma_{0} \leqslant t \leqslant 1-\sigma_{0} ;
$$

здесь и далее через $\Delta_{1, \varphi}, \Delta_{2, \varphi}$ и т. д. обозначаем остатки, модули которых имеют экспоненциальный порядок малости (т. е. порядок $\exp (-q / \varepsilon), q=$ const $>0$ ) равномерно по $\varphi, t$.

На следующем этапе, считая, что

$$
\sigma_{0}<\frac{1}{2}
$$

обратимся к отрезку $1-\sigma_{0} \leqslant t \leqslant 1+\sigma_{0}$. Так как в силу (2.17) его длина не превосходит единицы, то в этом случае $x_{\varphi}(t-1, \varepsilon)$ задается уже известным равенством (2.16) (при замене в нем $t$ на $t-1$ ). Тем самым, и здесь имеем дело с задачей Коши

$$
\dot{x}=F\left(t-1+\Delta_{1, \varphi}(t-1, \varepsilon), \varepsilon\right),\left.\quad x\right|_{t=1-\sigma_{0}}=1-\sigma_{0}+\Delta_{1, \varphi}\left(1-\sigma_{0}, \varepsilon\right),
$$

правые части которой известны из предыдущего шага. Что же касается решения этой задачи, то его будем искать в виде

$$
x_{\varphi}(t, \varepsilon)=1+\left.\varepsilon v(\tau)\right|_{\tau=(t-1) / \varepsilon}+\Delta_{2, \varphi}(t, \varepsilon), \quad 1-\sigma_{0} \leqslant t \leqslant 1+\sigma_{0},
$$

где

$$
v(\tau)=\tau+\int_{-\infty}^{\tau}[f(\exp s)-1] d s,
$$

a $\Delta_{2, \varphi}$ - подлежащий определению остаток. 
Подставляя выражения (2.19), (2.20) в $(2.18)$, для $\Delta_{2, \varphi}$ приходим к задаче Коши

$$
\dot{\Delta}_{2, \varphi}=G_{\varphi}(t, \varepsilon),\left.\quad \Delta_{2, \varphi}\right|_{t=1-\sigma_{0}}=\mathrm{g}_{\varphi}(\varepsilon),
$$

где

$$
\begin{aligned}
& G_{\varphi}(t, \varepsilon)=f\left(x_{1}\right)-f\left(x_{2}\right) \\
& \quad \text { при } x_{1}=\exp \left(\frac{t-1}{\varepsilon}+\frac{\Delta_{1, \varphi}(t-1, \varepsilon)}{\varepsilon}\right), \quad x_{2}=\exp \left(\frac{t-1}{\varepsilon}\right) ; \\
& \mathrm{g}_{\varphi}(\varepsilon)=-\sigma_{0}-\left.\varepsilon v(\tau)\right|_{\tau=-\sigma_{0} / \varepsilon}+\Delta_{1, \varphi}\left(1-\sigma_{0}, \varepsilon\right) .
\end{aligned}
$$

Таким образом, доказательство равномерной по $\varphi, t$ экспоненциальной малости $\Delta_{2, \varphi}$ сводится к проверке аналогичных свойств для функции $(2.22)$ и начального условия (2.23).

Обратимся сначала к анализу $\mathrm{g}_{\varphi}(\varepsilon)$. Учитывая в $(2.20)$ свойство $f(0)=1$, убеждаемся, что

$$
v(\tau)=\tau+O(\exp \tau), \quad \tau \rightarrow-\infty
$$

и, следовательно,

$$
-\sigma_{0}-\left.\varepsilon v(\tau)\right|_{\tau=-\sigma_{0} / \varepsilon}=O\left(\exp \left(-\frac{\sigma_{0}}{\varepsilon}\right)\right)
$$

А отсюда и из известных свойств $\Delta_{1, \varphi}$ вытекает требуемая экспоненциальная малость величины (2.23).

Для оценки функции (2.22) привлечем свойства (1.50) функции $f(u)$. В peзультате приходим к выводу, что

$$
M_{1}=\sup _{u \in \mathbb{R}_{+}}\left(1+u^{2}\right)\left|f^{\prime}(u)\right|<\infty .
$$

Далее, используя этот факт, нетрудно увидеть, что

$$
\left|f\left(u_{1}\right)-f\left(u_{2}\right)\right| \leqslant \frac{M_{1}}{1+\min \left(u_{1}^{2}, u_{2}^{2}\right)}\left|u_{1}-u_{2}\right| \quad \forall u_{1}, u_{2} \in \mathbb{R}_{+} .
$$

Применяя затем свойство (2.25) к правой части из (2.22), получаем неравенство

$$
\left|G_{\varphi}(t, \varepsilon)\right| \leqslant \frac{M_{2}}{\varepsilon} \exp \left(-\frac{|t-1|}{\varepsilon}\right)\left|\Delta_{1, \varphi}(t-1, \varepsilon)\right|, \quad 1-\sigma_{0} \leqslant t \leqslant 1+\sigma_{0},
$$

где $M_{2}$ - некоторая универсальная (не зависящая от $t, \varphi, \varepsilon$ ) положительная константа. Остается заметить, что из (2.26) равномерная по $t, \varphi$ экспоненциальная малость $G_{\varphi}(t, \varepsilon)$ следует уже очевидным образом.

Дальнейший анализ связан с рассмотрением отрезка $1+\sigma_{0} \leqslant t \leqslant 2+\sigma_{0}$ при условии

$$
\sigma_{0}<\frac{1}{2 a}
$$


гарантирующем выполнение неравенства $2+\sigma_{0}<t_{0}+1-\sigma_{0}$, где, напомним, $t_{0}-$ момент времени (2.9). В этом случае на промежутках $t \in\left[1+\sigma_{0}, 2-\sigma_{0}\right]$ и $t \in\left[2-\sigma_{0}, 2+\sigma_{0}\right]$ функция $x_{\varphi}(t-1, \varepsilon)$ задается соответственно формулами $(2.16)$ и $(2.19)$ (с заменой в них $t$ на $t-1)$. А отсюда, в частности, следует, что

$$
x_{\varphi}(t-1, \varepsilon) \geqslant q, \quad t \in\left[1+\sigma_{0}, 2-\sigma_{0}\right],
$$

где $q$ - произвольно фиксированная постоянная из интервала $\left(0, \sigma_{0}\right)$.

Для получения аналогичной (2.28) оценки на отрезке $2-\sigma_{0} \leqslant t \leqslant 2+\sigma_{0}$ необходимо наряду с (2.24) знать поведение функции (2.20) при $\tau \rightarrow+\infty$. Несложный подсчет показывает, что

$$
v(\tau)=-a \tau+c_{0}+O(\exp (-\tau)), \quad \tau \rightarrow+\infty,
$$

где

$$
c_{0}=\int_{0}^{1} \frac{f(u)-1}{u} d u+\int_{1}^{+\infty} \frac{f(u)+a}{u} d u .
$$

Учитывая, далее, соотношения (2.24), (2.29), (2.30) в (2.19), приходим к выводу, что

$$
x_{\varphi}(t-1, \varepsilon) \geqslant q, \quad t \in\left[2-\sigma_{0}, 2+\sigma_{0}\right],
$$

где теперь $q \in\left(0, \min \left(1-\sigma_{0}, 1-a \sigma_{0}\right)\right)$.

Установленные выше неравенства $(2.28),(2.31)$ и свойства (1.50) функции $f(u)$ позволяют заключить, что равномерно по $t \in\left[1+\sigma_{0}, 2+\sigma_{0}\right], \varphi \in S\left(\sigma_{0}, q_{1}, q_{2}\right)$ справедливо аналогичное (2.15) асимптотическое представление

$$
F\left(x_{\varphi}(t-1, \varepsilon), \varepsilon\right)=-a+O\left(\exp \left(-\frac{q}{\varepsilon}\right)\right), \quad q=\text { const }>0, \quad \varepsilon \rightarrow 0 .
$$

Подставляя его вместе с формулами (2.19), (2.20), (2.29), (2.30) в интегральное соотношение

$$
x_{\varphi}(t, \varepsilon)=\left.x_{\varphi}\right|_{t=1+\sigma_{0}}+\int_{1+\sigma_{0}}^{t} F\left(x_{\varphi}(s-1, \varepsilon), \varepsilon\right) d s,
$$

убеждаемся, что

$$
x_{\varphi}(t, \varepsilon)=1-a(t-1)+\varepsilon c_{0}+\Delta_{3, \varphi}(t, \varepsilon),
$$

где, как обычно, остаток $\Delta_{3, \varphi}$ равномерно по $\varphi, t$ экспоненциально мал.

Добавим еще, что на самом деле формула (2.34) сохраняется (вместе с требуемой малостью остатка) и на отрезке $2+\sigma_{0} \leqslant t \leqslant t_{0}+1-\sigma_{0}$. Обосновать этот факт позволяет так называемый метод шагов, суть которого состоит в следующем. Разобьем указанный отрезок на части длины не более единицы и на очередном промежутке, опираясь на формулу (2.34) (уже установленную на предыдущем участке изменения $t$ ), сначала получаем оценку вида $x_{\varphi}(t-1, \varepsilon) \geqslant q$ с некоторой универсальной постоянной $q>0$, а затем распространяем равенство (2.34) на шаг вперед. Ясно, что описанный процесс продолжается до тех пор, пока $1-a(t-2)>0$. Тем самым, рассуждая подобным образом, мы заведомо добираемся до точки $t=t_{0}+1-\sigma_{0}$. 
На очередном отрезке $t_{0}+1-\sigma_{0} \leqslant t \leqslant t_{0}+1+\sigma_{0}$ в силу $(2.3),(2.34)$ и оценок $(2.17),(2.27)$ интересующее нас решение $x_{\varphi}(t, \varepsilon)$ определяется из задачи Коши

$$
\begin{gathered}
\dot{x}=F\left(-a\left(t-t_{0}-1\right)+\varepsilon c_{0}+\Delta_{3, \varphi}(t-1, \varepsilon), \varepsilon\right), \\
\left.x\right|_{t=t_{0}+1-\sigma_{0}}=-a\left(1-\sigma_{0}\right)+\varepsilon c_{0}+\Delta_{3, \varphi}\left(t_{0}+1-\sigma_{0}, \varepsilon\right) .
\end{gathered}
$$

Решение получившейся задачи будем искать в виде

$$
\begin{gathered}
x_{\varphi}(t, \varepsilon)=-a+\left.\varepsilon w(\tau)\right|_{\tau=\left(t-t_{0}-1\right) / \varepsilon}+\Delta_{4, \varphi}(t, \varepsilon), \\
t_{0}+1-\sigma_{0} \leqslant t \leqslant t_{0}+1+\sigma_{0},
\end{gathered}
$$

где

$$
w(\tau)=-a \tau+c_{0}+\int_{-\infty}^{\tau}\left[f\left(\exp \left(-a s+c_{0}\right)\right)+a\right] d s .
$$

В результате для очередного остатка $\Delta_{4, \varphi}$ выходит аналогичная (2.21) задача Коши

$$
\dot{\Delta}_{4, \varphi}=G_{\varphi}(t, \varepsilon),\left.\quad \Delta_{4, \varphi}\right|_{t=t_{0}+1-\sigma_{0}}=\mathrm{g}_{\varphi}(\varepsilon)
$$

где теперь

$$
\begin{gathered}
G_{\varphi}(t, \varepsilon)=f\left(x_{1}\right)-f\left(x_{2}\right) \\
\text { при } x_{1}=\exp \left(-\frac{a\left(t-t_{0}-1\right)}{\varepsilon}+c_{0}+\frac{\Delta_{3, \varphi}(t-1, \varepsilon)}{\varepsilon}\right), \\
x_{2}=\exp \left(-\frac{a\left(t-t_{0}-1\right)}{\varepsilon}+c_{0}\right) ; \\
\mathrm{g}_{\varphi}(\varepsilon)=a \sigma_{0}+\varepsilon c_{0}-\left.\varepsilon w(\tau)\right|_{\tau=-\sigma_{0} / \varepsilon}+\Delta_{3, \varphi}\left(t_{0}+1-\sigma_{0}, \varepsilon\right) .
\end{gathered}
$$

Оценка правых частей из (2.37) проводится по той же схеме, что и в случае (2.22), (2.23). Действительно, привлекая вытекающее из (2.36) асимптотическое равенство

$$
w(\tau)=-a \tau+c_{0}+O(\exp (a \tau)), \quad \tau \rightarrow-\infty,
$$

приходим к выводу, что

$$
a \sigma_{0}+\varepsilon c_{0}-\left.\varepsilon w(\tau)\right|_{\tau=-\sigma_{0} / \varepsilon}=O\left(\exp \left(-\frac{a \sigma_{0}}{\varepsilon}\right)\right), \quad \varepsilon \rightarrow 0 .
$$

А отсюда с учетом уже установленных свойств $\Delta_{3, \varphi}$ следует нужная экспоненциальная малость начального условия (2.39). Что же касается функции (2.38), то проверка ее равномерной по $t, \varphi$ экспоненциальной малости базируется на неравенстве (2.25) (см. аналогичное место выше).

Перейдем, далее, к отрезку $t_{0}+1+\sigma_{0} \leqslant t \leqslant \min \left(t_{0}+2+\sigma_{0}, T_{0}+\sigma_{0}\right)$. Так как его длина не превосходит единицы, то при указанных $t$ функция $x_{\varphi}(t-1, \varepsilon)$ уже известна и задается равенствами (2.34) или (2.35) (при замене в них $t$ на $t-1)$. В обоих случаях, однако, справедлива оценка вида

$$
x_{\varphi}(t-1, \varepsilon) \leqslant-q
$$

с некоторой универсальной положительной константой $q$. 
Действительно, при тех значениях $t$, когда работает формула (2.34), в качестве $q$ в (2.41) можно взять любое число из интервала $\left(0, a \sigma_{0}\right)$. В случае же, когда имеем дело с формулой (2.35), для оценки $q$ кроме свойства $(2.40)$ необходимо знать асимптотическое поведение функции (2.36) при $\tau \rightarrow+\infty$. Соответствующую асимптотическую формулу легко вывести, исходя из представления

$$
\begin{aligned}
w(\tau)=\tau & -\frac{c_{0}}{a}+\int_{-\infty}^{c_{0} / a}\left[f\left(\exp \left(-a s+c_{0}\right)\right)+a\right] d s \\
& +\int_{c_{0} / a}^{+\infty}\left[f\left(\exp \left(-a s+c_{0}\right)\right)-1\right] d s-\int_{\tau}^{+\infty}\left[f\left(\exp \left(-a s+c_{0}\right)\right)-1\right] d s .
\end{aligned}
$$

Для этого достаточно заметить, что последнее слагаемое в $(2.42)$ при $\tau \rightarrow+\infty$ имеет порядок $\exp (-a \tau)$, а сумма

$$
\begin{gathered}
\int_{-\infty}^{c_{0} / a}\left[f\left(\exp \left(-a s+c_{0}\right)\right)+a\right] d s+\int_{c_{0} / a}^{+\infty}\left[f\left(\exp \left(-a s+c_{0}\right)\right)-1\right] d s \\
=\frac{1}{a}\left(\int_{0}^{1} \frac{f(u)-1}{u} d u+\int_{1}^{+\infty} \frac{f(u)+a}{u} d u\right)
\end{gathered}
$$

в силу (2.30) совпадает с $c_{0} / a$. Таким образом, из (2.42) окончательно получаем

$$
w(\tau)=\tau+O(\exp (-a \tau)), \quad \tau \rightarrow+\infty .
$$

Возвратимся к оценке (2.41). Из (2.35), (2.40), (2.43) следует, что в случае, когда $x_{\varphi}(t-1, \varepsilon)$ задается с помощью формулы $(2.35)$, в качестве $q$ в $(2.41)$ можно взять любое число из интервала $\left(0, \min \left(a-\sigma_{0}, a-a \sigma_{0}\right)\right)$.

Установленное выше неравенство (2.41) и свойства (1.49) приводят к асимптотическому представлению

$$
F\left(x_{\varphi}(t-1, \varepsilon), \varepsilon\right)=1+O\left(\exp \left(-\frac{q}{\varepsilon}\right)\right), \quad q=\text { const }>0, \quad \varepsilon \rightarrow 0,
$$

которое, как и аналогичные ему соотношения (2.15), (2.32), справедливо равномерно по $\varphi, t$. Дальнейший же способ действий стандартен: подставляем (2.44) вместе с формулами (2.35), (2.36), (2.43) в аналогичное (2.33) интегральное уравнение

$$
x_{\varphi}(t, \varepsilon)=\left.x_{\varphi}\right|_{t=t_{0}+1+\sigma_{0}}+\int_{t_{0}+1+\sigma_{0}}^{t} F\left(x_{\varphi}(s-1, \varepsilon), \varepsilon\right) d s
$$

и после несложных выкладок приходим к очередному асимптотическому равенству

$$
x_{\varphi}(t, \varepsilon)=t-T_{0}+\Delta_{5, \varphi}(t, \varepsilon),
$$

где остаток $\Delta_{5, \varphi}$ обладает требуемой экспоненциальной малостью.

В случае, когда $t_{0}+2<T_{0}$, отдельному рассмотрению подлежит еще отрезок $t_{0}+2+\sigma_{0} \leqslant t \leqslant T_{0}+\sigma_{0}$. Однако здесь не возникают новые моменты, так как 
при указанных $t$ сохраняется априорная оценка (2.41) (см. аналогичное место при обосновании формулы (2.34)). Тем самым, асимптотическое представление (2.46) оказывается пригодным на всем отрезке $t_{0}+1+\sigma_{0} \leqslant t \leqslant T_{0}+\sigma_{0}$.

Подведем некоторый итог. Из (2.44)-(2.46) очевидным образом следует, что интересующий нас корень $t=T_{\varphi}$ уравнения $x_{\varphi}\left(t-\sigma_{0}, \varepsilon\right)=-\sigma_{0}$ определяется однозначно, причем равномерно по $\varphi \in S\left(\sigma_{0}, q_{1}, q_{2}\right)$

$$
T_{\varphi}=T_{0}+O\left(\exp \left(-\frac{q}{\varepsilon}\right)\right), \quad q=\text { const }>0, \quad \varepsilon \rightarrow 0 .
$$

Далее, объединяя все полученные выше асимптотические представления для решения $x_{\varphi}(t, \varepsilon)$ (см. $\left.(2.16),(2.19),(2.34),(2.35),(2.46)\right)$, приходим к выводу, что

$$
\max _{-\sigma_{0} \leqslant t \leqslant T_{0}+\sigma_{0}}\left|x_{\varphi}(t, \varepsilon)-x_{0}(t)\right|=O(\varepsilon), \quad \varepsilon \rightarrow 0,
$$

где $x_{0}(t)$ - функция (2.11), а остаток равномерен по $\varphi \in S\left(\sigma_{0}, q_{1}, q_{2}\right)$.

Формулы $(2.47),(2.48)$ свидетельствуют о том, что оператор (2.14) действительно определен на множестве $S\left(\sigma_{0}, q_{1}, q_{2}\right)$ и равномерно по $\varphi$

$$
\max _{-1-\sigma_{0} \leqslant t \leqslant-\sigma_{0}}\left|x_{\varphi}\left(t+T_{\varphi}, \varepsilon\right)-x_{0}(t)\right|=O(\varepsilon), \quad \varepsilon \rightarrow 0 .
$$

Что же касается требуемого включения $\Pi_{\varepsilon}\left(S\left(\sigma_{0}, q_{1}, q_{2}\right)\right) \subset S\left(\sigma_{0}, q_{1}, q_{2}\right)$, то в силу (2.49) оно будет заведомо выполняться для всех достаточно малых $\varepsilon>0$ при условии

$$
x_{0}(t) \in \widehat{S}\left(\sigma_{0}, q_{1}, q_{2}\right),
$$

где $\widehat{S}\left(\sigma_{0}, q_{1}, q_{2}\right)$ - множество функций, получающееся из $S\left(\sigma_{0}, q_{1}, q_{2}\right)$ при замене в (2.13) нестрогих неравенств строгими. Напомним, далее, что на параметр $\sigma_{0}$ нами уже наложено ограничение $(2.5)$, обеспечивающее свойства $x_{0}\left(-\sigma_{0}\right)=-\sigma_{0}$ и $x_{0}(t)<0$ при $-1-\sigma_{0} \leqslant t \leqslant-\sigma_{0}$. Поэтому справедливости включения $(2.50)$ добиваемся за счет имеющихся в запасе параметров $q_{1}, q_{2}$, предполагая, что

$$
q_{1}>-\min _{-1-\sigma_{0} \leqslant t \leqslant-\sigma_{0}} x_{0}(t), \quad 0<q_{2}<-\max _{-1-\sigma_{0} \leqslant t \leqslant-\sigma_{0}} x_{0}(t) .
$$

Итак, оператор $\Pi_{\varepsilon}$, являющийся очевидным образом компактным, при выполнении условий $(2.5),(2.17),(2.27),(2.51)$ на параметры $\sigma_{0}, q_{1}, q_{2}$ преобразует в себя замкнутое, ограниченное и выпуклое множество $S\left(\sigma_{0}, q_{1}, q_{2}\right)$. А отсюда в соответствии с известным принципом Шаудера заключаем, что этот оператор имеет в $S\left(\sigma_{0}, q_{1}, q_{2}\right)$ по крайней мере одну неподвижную точку $\varphi=\varphi_{*}(t, \varepsilon)$. Ясно также, что решение $x_{*}(t, \varepsilon)$ уравнения $(2.3)$ с начальной функцией $\varphi_{*}(t, \varepsilon)$, $-1-\sigma_{0} \leqslant t \leqslant-\sigma_{0}$, оказывается периодическим с периодом $T_{*}(\varepsilon)=\left.T_{\varphi}\right|_{\varphi=\varphi_{*}}$ и в силу (2.47)-(2.49) обладает требуемыми свойствами (2.12).

2.3. Анализ свойств устойчивости. Перейдем теперь ко второй части обоснования теоремы 2.1, т. е. к доказательству единственности и устойчивости релаксационного цикла $x_{*}(t, \varepsilon)$ с нулевым приближением (2.11). Из явной формулы (2.14) для оператора $\Pi_{\varepsilon}$ вытекает, что он непрерывно дифференцируем по $\varphi$, а его производная Фреше $\partial_{\varphi} \Pi_{\varepsilon}(\varphi)$ задается равенством

$$
\partial_{\varphi} \Pi_{\varepsilon}(\varphi) \mathrm{g}_{0}=\mathrm{g}\left(t+T_{\varphi}, \varepsilon\right)-l\left(\mathrm{~g}_{0}\right) \dot{x}_{\varphi}\left(t+T_{\varphi}, \varepsilon\right), \quad-1-\sigma_{0} \leqslant t \leqslant-\sigma_{0} .
$$


Здесь функция $\mathrm{g}_{0}(t)$ представляет собой произвольный элемент пространства

$$
C_{0}=\left\{\mathrm{g}_{0}(t) \in C\left[-1-\sigma_{0},-\sigma_{0}\right]: \mathrm{g}_{0}\left(-\sigma_{0}\right)=0\right\},
$$

через $\mathrm{g}(t, \varepsilon),-\sigma_{0} \leqslant t \leqslant T_{\varphi}-\sigma_{0}$, обозначено решение линейного уравнения

$$
\dot{\mathrm{g}}=A(t, \varepsilon) \mathrm{g}(t-1), \quad A(t, \varepsilon)=\left.\frac{1}{\varepsilon}\left(f^{\prime}(\exp x) \exp x\right)\right|_{x=x_{\varphi}(t-1, \varepsilon) / \varepsilon}
$$

с начальной функцией $\mathrm{g}_{0}(t),-1-\sigma_{0} \leqslant t \leqslant-\sigma_{0}$, а линейный функционал $l: C_{0} \rightarrow \mathbb{R}$ определен формулой

$$
l\left(\mathrm{~g}_{0}\right)=\frac{\mathrm{g}\left(T_{\varphi}-\sigma_{0}, \varepsilon\right)}{\dot{x}_{\varphi}\left(T_{\varphi}-\sigma_{0}, \varepsilon\right)} .
$$

Из соотношений (2.52), (2.54) следует, что проблема оценки нормы линейного оператора $\partial_{\varphi} \Pi_{\varepsilon}(\varphi)$ в пространстве $C_{0}$ с нормой

$$
\left\|\mathrm{g}_{0}\right\|=\max _{-1-\sigma_{0} \leqslant t \leqslant-\sigma_{0}}\left|\mathrm{~g}_{0}(t)\right|
$$

сводится к анализу введенного выше решения $\mathrm{g}(t, \varepsilon)$ уравнения (2.53). Покажем, что для этого решения выполняется неравенство вида

$$
\max _{-\sigma_{0} \leqslant t \leqslant T_{\varphi}-\sigma_{0}}|\mathrm{~g}(t, \varepsilon)| \leqslant M \exp \left(-\frac{q}{\varepsilon}\right)\left\|\mathrm{g}_{0}\right\|
$$

с некоторыми универсальными (не зависящими от $\varepsilon, \varphi, \mathrm{g}_{0}$ ) положительными постоянными $M, q$.

Обратимся сначала к отрезку $-\sigma_{0} \leqslant t \leqslant 1-\sigma_{0}$, на котором из $(2.53)$ для $\mathrm{g}(t, \varepsilon)$ имеем явную формулу

$$
\mathrm{g}(t, \varepsilon)=\int_{-\sigma_{0}}^{t} A(s, \varepsilon) \mathrm{g}_{0}(s-1) d s, \quad-\sigma_{0} \leqslant t \leqslant 1-\sigma_{0} .
$$

Напомним, далее, что при рассматриваемых $t$ решение $x_{\varphi}(t-1, \varepsilon)$ совпадает с функцией $\varphi(t-1)$, удовлетворяющей условиям (2.13). А отсюда и из свойств (1.49) функции $f(u)$, в свою очередь, следует, что фигурирующий в $(2.53)$ коэффициент $A(t, \varepsilon)$ допускает оценку вида

$$
\max _{-\sigma_{0} \leqslant t \leqslant 1-\sigma_{0}}|A(t, \varepsilon)| \leqslant M \exp \left(-\frac{q}{\varepsilon}\right) .
$$

И наконец, учитывая (2.57) в (2.56), убеждаемся, что

$$
\max _{-\sigma_{0} \leqslant t \leqslant 1-\sigma_{0}}|\mathrm{~g}(t, \varepsilon)| \leqslant M \exp \left(-\frac{q}{\varepsilon}\right)\left\|\mathrm{g}_{0}\right\| .
$$


Для распространения оценки (2.58) на оставшийся отрезок $\left[1-\sigma_{0}, T_{\varphi}-\sigma_{0}\right]$ изменения $t$ воспользуемся методом шагов. А именно, разобьем указанный промежуток времени на отрезки $\left[1-\sigma_{0}+k, 2-\sigma_{0}+k\right], k=0,1, \ldots, k_{0}$, и $\left[2-\sigma_{0}+k_{0}, T_{\varphi}-\sigma_{0}\right]$, где $k_{0}=\left\lfloor T_{\varphi}-2\right\rfloor,\lfloor\cdot\rfloor$ - целая часть. Опираясь, далее, на равномерную по $\varphi \in S\left(\sigma_{0}, q_{1}, q_{2}\right)$ асимптотическую формулу

$$
\max _{1-\sigma_{0} \leqslant t \leqslant T_{\varphi}-\sigma_{0}}|A(t, \varepsilon)|=O\left(\frac{1}{\varepsilon}\right), \quad \varepsilon \rightarrow 0,
$$

замечаем, что из неравенства

$$
|\mathrm{g}(t, \varepsilon)| \leqslant\left|\mathrm{g}\left(1-\sigma_{0}+k, \varepsilon\right)\right|+\int_{1-\sigma_{0}+k}^{t}|A(s, \varepsilon)| \cdot|\mathrm{g}(s-1, \varepsilon)| d s, \quad t \geqslant 1-\sigma_{0}+k,
$$

и из уже полученной оценки вида $(2.58)$ на $(k-1)$-м отрезке вытекает требуемая оценка на $k$-м отрезке изменения $t$.

Возвращаясь к оператору $\Pi_{\varepsilon}$ и учитывая установленное выше неравенство $(2.55)$ в $(2.52),(2.54)$, приходим к выводу, что

$$
\sup _{\varphi \in S\left(\sigma_{0}, q_{1}, q_{2}\right)}\left\|\partial_{\varphi} \Pi_{\varepsilon}(\varphi)\right\|_{C_{0} \rightarrow C_{0}} \leqslant M \exp \left(-\frac{q}{\varepsilon}\right) .
$$

Остается добавить, что оценка (2.59) обеспечивает как сжимаемость оператора $\Pi_{\varepsilon}$ (а значит, единственность его неподвижной точки $\varphi=\varphi_{*}(t, \varepsilon)$ в множестве $\left.S\left(\sigma_{0}, q_{1}, q_{2}\right)\right)$, так и экспоненциальную орбитальную устойчивость соответствующего цикла $x_{*}(t, \varepsilon)$. Теорема 2.1 полностью доказана.

В дополнение к установленной теореме отметим, что релаксационный цикл $u_{*}(t, \lambda), u_{*}(0, \lambda) \equiv 1$, исходного уравнения (1.51) задается равенством

$$
u_{*}(t, \lambda)=\left.\exp \left(\frac{x_{*}\left(t+t_{*}(\varepsilon), \varepsilon\right)}{\varepsilon}\right)\right|_{\varepsilon=1 / \lambda},
$$

где $t=t_{*}(\varepsilon)$ - корень уравнения $x_{*}(t, \varepsilon)=0$, лежащий в асимптотически малой окрестности точки $t=0$. Из формул $(2.15),(2.16)$ следует, что этот корень определяется однозначно и допускает асимптотику $t_{*}(\varepsilon)=O(\exp (-q / \varepsilon)), \varepsilon \rightarrow 0$, где $q=$ const $>0$. Что же касается самого цикла $(2.60)$, то в силу $(2.47),(2.48)$ он обладает требуемыми асимптотическими характеристиками (2.1), (2.2).

Завершая рассмотрение уравнения (1.51), остановимся на нейродинамической интерпретации полученных нами результатов. В первую очередь отметим, что функционирование нейрона в режиме устойчивого цикла (2.60) соответствует режиму генерации спайков. Спайком в данном случае уместно назвать промежуток времени, на котором функция (2.60) принимает асимптотически большие значения, т. е. участок всплеска. В силу (2.60), (2.48), (2.11) этот участок соответствует значениям $0 \lesssim t \lesssim t_{0}$. Оставшийся же промежуток $t_{0} \lesssim t \lesssim T_{0}$ представляет собой участок рефрактерности. Из биологических соображений ясно, что длительность спайка должна быть меньше длительности рефрактерности, а значит, должно выполняться неравенство $t_{0}<T_{0}-t_{0}$. Привлекая, далее, явные формулы для $t_{0}, T_{0}$ (см. $\left.(2.1),(2.9)\right)$, убеждаемся, что данное неравенство эквивалентно условию

$$
a>1 \text {. }
$$

Ниже при рассмотрении различных цепочек связанных уравнений вида (1.51) это условие в некоторых случаях будем считать выполненным. 


\section{3. Автоволновые процессы в сетях связанных нейронов}

3.1. Базовые теоремы. В данном разделе исследованию подлежат аналогичные (1.36)-(1.38) системы электрически связанных нейронов, каждый из которых в отдельности моделируется уравнением (1.51), а также аналогичные (1.39) системы синаптически связанных нейронов.

Обратимся сначала к диффузионной нейронной сети, которая описывается системой вида

$$
\dot{u}_{j}=d\left(u_{j+1}-2 u_{j}+u_{j-1}\right)+\lambda f\left(u_{j}(t-1)\right) u_{j}, \quad j=1, \ldots, m, \quad m \geqslant 2 .
$$

Здесь $u_{0}=u_{1}, u_{m+1}=u_{m}, \lambda \gg 1$, а параметр $d>0$ имеет порядок единицы. Что же касается функции $f(u)$, то она удовлетворяет прежним ограничениям (1.49), (1.50) и дополнительному требованию (2.61).

Система (3.1) допускает, очевидно, так называемый однородный или синхронный цикл

$$
u_{1} \equiv \cdots \equiv u_{m}=u_{*}(t, \lambda),
$$

где $u_{*}(t, \lambda)$ - устойчивое периодическое решение (2.60) уравнения (1.51). Наш основной результат состоит в том, что при подходящем уменьшении $d$ и при всех $\lambda \gg 1$ эта система наряду с устойчивым циклом (3.2) имеет не менее $m$ экспоненциально орбитально устойчивых неоднородных периодических движений, которые по аналогии с пространственно непрерывным случаем будем называть автоволновыми процессами. А так как размерность $m$ системы (3.1) мы можем выбирать сколь угодно большой, то это означает реализуемость в рассматриваемой нейронной модели феномена буферности.

Для удобства дальнейшего асимптотического анализа перейдем в (3.1) к новым переменным $x, y_{1}, \ldots, y_{m-1}$ по формулам

$$
u_{1}=\exp \left(\frac{x}{\varepsilon}\right), \quad u_{j}=\exp \left(\frac{x}{\varepsilon}+\sum_{k=1}^{j-1} y_{k}\right), \quad j=2, \ldots, m, \quad \varepsilon=\frac{1}{\lambda} .
$$

В результате получаем систему

$$
\begin{aligned}
\dot{x}= & \varepsilon d\left(\exp y_{1}-1\right)+F(x(t-1), \varepsilon), \\
\dot{y}_{j}=d[ & \left.\exp y_{j+1}+\exp \left(-y_{j}\right)-\exp y_{j}-\exp \left(-y_{j-1}\right)\right] \\
& \quad+G_{j}\left(x(t-1), y_{1}(t-1), \ldots, y_{j}(t-1), \varepsilon\right), \quad j=1, \ldots, m-1,
\end{aligned}
$$

где $y_{0}=y_{m}=0$, функция $F(x, \varepsilon)$ та же самая, что и в $(2.3)$, а нелинейности $G_{j}$ задаются соотношениями

$$
\begin{gathered}
G_{j}\left(x, y_{1}, \cdots, y_{j}, \varepsilon\right)=\frac{1}{\varepsilon}\left\{f\left(\exp \left(\frac{x}{\varepsilon}+\sum_{k=1}^{j} y_{k}\right)\right)-f\left(\exp \left(\frac{x}{\varepsilon}+\sum_{k=1}^{j-1} y_{k}\right)\right)\right\}, \\
j=1, \ldots, m-1 .
\end{gathered}
$$

Зафиксируем постоянную $\sigma_{0}$, подчиненную требованиям $0<\sigma_{0}<a-1$, и рассмотрим банахово пространство $\mathscr{F}$ непрерывных при $-1-\sigma_{0} \leqslant t \leqslant-\sigma_{0}$ вектор-функций $\varphi(t)=\left(\varphi_{1}(t), \ldots, \varphi_{m}(t)\right)$ с нормой

$$
\|\varphi\|_{\mathscr{F}}=\max _{1 \leqslant j \leqslant m}\left(\max _{-1-\sigma_{0} \leqslant t \leqslant-\sigma_{0}}\left|\varphi_{j}(t)\right|\right) .
$$


Всюду ниже нас будут интересовать решения системы (3.4), (3.5) с начальными условиями из множества

$$
S=\left\{\varphi(t)=\left(\varphi_{1}(t), \ldots, \varphi_{m}(t)\right): \varphi_{1} \in S_{1}, \varphi_{2} \in S_{2}, \ldots, \varphi_{m} \in S_{m}\right\} \subset \mathscr{F} .
$$

Здесь через $S_{1}$ обозначена введенная при доказательстве теоремы 2.1 совокупность непрерывных функций $S\left(\sigma_{0}, q_{1}, q_{2}\right)$, параметры $q_{1}, q_{2}$ в которой удовлетворяют неравенствам (2.51), а в качестве $S_{2}, \ldots, S_{m}$ взяты произвольные замкнутые и ограниченные подмножества пространства $C\left[-1-\sigma_{0},-\sigma_{0}\right]$.

Формулировка строгих результатов об автоволновых режимах системы (3.4), (3.5) требует некоторых подготовительных построений. В связи с этим введем в рассмотрение решение

$$
\left(x_{\varphi}(t, \varepsilon), y_{1, \varphi}(t, \varepsilon), \ldots, y_{m-1, \varphi}(t, \varepsilon)\right), \quad t \geqslant-\sigma_{0},
$$

упомянутой системы, отвечающее произвольному начальному условию $\varphi(t)$ из множества $S$. Рассмотрим также второй положительный корень $t=T_{\varphi}$ уравнения $x_{\varphi}\left(t-\sigma_{0}, \varepsilon\right)=-\sigma_{0}$ (в случае, когда он существует) и на множестве $(3.8)$ определим оператор $\Pi_{\varepsilon}: S \rightarrow \mathscr{F}$ посредством аналогичного (2.14) равенства

$$
\begin{gathered}
\Pi_{\varepsilon}(\varphi)=\left(x_{\varphi}\left(t+T_{\varphi}, \varepsilon\right), y_{1, \varphi}\left(t+T_{\varphi}, \varepsilon\right), \ldots, y_{m-1, \varphi}\left(t+T_{\varphi}, \varepsilon\right)\right), \\
-1-\sigma_{0} \leqslant t \leqslant-\sigma_{0} .
\end{gathered}
$$

Помимо (3.10) нам потребуется еще оператор $\Pi_{0}: S \rightarrow \mathscr{F}$, который зададим формулой

$$
\begin{gathered}
\Pi_{0}(\varphi)=\left.\left(x_{0}(t), y_{1}^{0}\left(t+T_{0}, z\right), \ldots, y_{m-1}^{0}\left(t+T_{0}, z\right)\right)\right|_{z=\left(\varphi_{2}\left(-\sigma_{0}\right), \ldots, \varphi_{m}\left(-\sigma_{0}\right)\right)}, \\
-1-\sigma_{0} \leqslant t \leqslant-\sigma_{0} .
\end{gathered}
$$

Здесь, как и ранее, $T_{0}$ - величина из $(2.1)$, а $x_{0}(t)$ - периодическая функция, определенная в (2.11). Что же касается компонент $y_{1}^{0}(t, z), \ldots, y_{m-1}^{0}(t, z)$, зависящих от вектора $z=\left(z_{1}, \ldots, z_{m-1}\right) \in \mathbb{R}^{m-1}$, то при $-1-\sigma_{0} \leqslant t \leqslant T_{0}-\sigma_{0}$ они удовлетворяют импульсной задаче Коши

$$
\begin{gathered}
\dot{y}_{j}=d\left[\exp y_{j+1}+\exp \left(-y_{j}\right)-\exp y_{j}-\exp \left(-y_{j-1}\right)\right], \\
y_{j}(1+0)=y_{j}(1-0)-(1+a) y_{j}(0), \\
y_{j}\left(t_{0}+1+0\right)=y_{j}\left(t_{0}+1-0\right)-\left(1+\frac{1}{a}\right) y_{j}\left(t_{0}\right), \\
j=1, \ldots, m-1, \quad y_{0}=y_{m}=0, \\
\left.\left(y_{1}, \ldots, y_{m-1}\right)\right|_{t=-\sigma_{0}}=\left(z_{1}, \ldots, z_{m-1}\right),
\end{gathered}
$$

где $t_{0}$ - момент времени (2.9).

Отдельно остановимся на вопросе о корректности определения оператора (3.11). Главная проблема здесь заключается в том, что на промежутках времени $-\sigma_{0} \leqslant t<1,1 \leqslant t<t_{0}+1$ и $t_{0}+1 \leqslant t \leqslant T_{0}-\sigma_{0}$ решение задачи Коши (3.12), (3.13) удовлетворяет нелинейной системе

$$
\begin{gathered}
\dot{y}_{j}=d\left[\exp y_{j+1}+\exp \left(-y_{j}\right)-\exp y_{j}-\exp \left(-y_{j-1}\right)\right], \\
j=1, \ldots, m-1, \quad y_{0}=y_{m}=0 .
\end{gathered}
$$


Поэтому возникает вопрос о продолжимости решений последней на указанные промежутки, длины которых отнюдь не малы. Ответ на этот вопрос дается в следующем утверждении.

Лемма 3.1. Решение $\left(y_{1}(t), \ldots, y_{m-1}(t)\right)$ системы (3.14) с произвольным начальным условием $\left.\left(y_{1}, \ldots, y_{m-1}\right)\right|_{t=0}=\left(\bar{y}_{1}, \ldots, \bar{y}_{m-1}\right) \in \mathbb{R}^{m-1}$ определено на полуоси $t \geqslant 0$ и стремится $\kappa$ нулю при $t \rightarrow+\infty$.

Для доказательства заметим, что любое решение системы (3.14) записывается в виде

$$
y_{j}(t)=\ln \frac{\xi_{j+1}(t)}{\xi_{j}(t)}, \quad j=1, \ldots, m-1
$$

где $\left(\xi_{1}(t), \ldots, \xi_{m}(t)\right)$ - произвольное решение линейной системы

$$
\dot{\xi}_{j}=d\left(\xi_{j+1}-2 \xi_{j}+\xi_{j-1}\right), \quad j=1, \ldots, m, \quad \xi_{0}=\xi_{1}, \quad \xi_{m+1}=\xi_{m},
$$

принадлежащее инвариантному конусу $K=\left\{\left(\xi_{1}, \ldots, \xi_{m}\right): \xi_{j}>0, j=1, \ldots, m\right\}$.

Свойства системы (3.16) хорошо известны. Во-первых, все ее решения при $t \rightarrow+\infty$ стремятся к устойчивому одномерному инвариантному многообразию $\left\{\left(\xi_{1}, \ldots, \xi_{m}\right): \xi_{1}=\xi_{2}=\cdots=\xi_{m}=c, c \in \mathbb{R}\right\}$, движения на котором задаются уравнением $\dot{c}=0$. Во-вторых, справедлив закон сохранения $\sum_{j=1}^{m} \xi_{j} \equiv$ const. Далее, из упомянутых свойств следует, что для любого решения $\left(\xi_{1}(t), \ldots, \xi_{m}(t)\right) \in K$ этой системы выполняются предельные равенства

$$
\lim _{t \rightarrow+\infty} \xi_{1}(t)=\lim _{t \rightarrow+\infty} \xi_{2}(t)=\cdots=\lim _{t \rightarrow+\infty} \xi_{m}(t)=\frac{1}{m} \sum_{j=1}^{m} \xi_{j}(0)>0 .
$$

А отсюда и из (3.15) утверждение леммы 3.1 вытекает очевидным образом.

Другая проблема, связанная с корректностью оператора (3.11), состоит в том, что функции $y_{j}^{0}(t, z), j=1, \ldots, m-1$, являются разрывными в точках $t=1$ и $t=t_{0}+1$, где согласно (3.12) они претерпевают конечные скачки. Однако в силу неравенства $(2.61)$ и оценки $\sigma_{0}<a-1$ функции $y_{j}^{0}\left(t+T_{0}, z\right), j=1, \ldots, m-1$, оказываются непрерывными на нужном отрезке $-1-\sigma_{0} \leqslant t \leqslant-\sigma_{0}$, поскольку в этом случае $T_{0}-1-\sigma_{0}>t_{0}+1$. Тем самым, условие $(2.61)$, выведенное нами из нейродинамических соображений, приобретает дополнительный математический смысл: оно гарантирует выполнение требуемого включения $\Pi_{0}(\varphi) \in \mathscr{F}$ при всех $\varphi \in S$.

Завершая описание подготовительной части, рассмотрим производные Фреше $\partial_{\varphi} \Pi_{\varepsilon}(\varphi), \partial_{\varphi} \Pi_{0}(\varphi)$ операторов $(3.10),(3.11)$ по переменной $\varphi$. Проводя соответствующий подсчет, убеждаемся, что в данном случае эти производные представляют собой линейные операторы, действующие в пространстве

$$
\mathscr{F}_{0}=\left\{\mathrm{g}_{0}(t)=\left(\mathrm{g}_{1,0}(t), \mathrm{g}_{2,0}(t), \ldots, \mathrm{g}_{m, 0}(t)\right) \in \mathscr{F}: \mathrm{g}_{1,0}\left(-\sigma_{0}\right)=0\right\}
$$


с нормой (3.7), а результаты их применения к произвольному элементу $\mathrm{g}_{0}(t)$ из $\mathscr{F}_{0}$ задаются соответственно равенствами

$$
\begin{aligned}
& \partial_{\varphi} \Pi_{\varepsilon}(\varphi) \mathrm{g}_{0}=\left(\mathrm{g}_{1}\left(t+T_{\varphi}, \varepsilon\right), \ldots, \mathrm{g}_{m}\left(t+T_{\varphi}, \varepsilon\right)\right) \\
& \quad-l\left(\mathrm{~g}_{0}\right)\left(\dot{x}_{\varphi}\left(t+T_{\varphi}, \varepsilon\right), \dot{y}_{1, \varphi}\left(t+T_{\varphi}, \varepsilon\right), \ldots, \dot{y}_{m-1, \varphi}\left(t+T_{\varphi}, \varepsilon\right)\right) \\
& \quad-1-\sigma_{0} \leqslant t \leqslant-\sigma_{0} ; \\
& \partial_{\varphi} \Pi_{\varepsilon}(\varphi) \mathrm{g}_{0}=\left(0, \sum_{s=1}^{m-1} \frac{\partial y_{1}^{0}}{\partial z_{s}}\left(t+T_{0}, z\right) \mathrm{g}_{s+1,0}\left(-\sigma_{0}\right),\right. \\
& \left.\quad \sum_{s=1}^{m-1} \frac{\partial y_{2}^{0}}{\partial z_{s}}\left(t+T_{0}, z\right) \mathrm{g}_{s+1,0}\left(-\sigma_{0}\right), \ldots, \sum_{s=1}^{m-1} \frac{\partial y_{m-1}^{0}}{\partial z_{s}}\left(t+T_{0}, z\right) \mathrm{g}_{s+1,0}\left(-\sigma_{0}\right)\right), \\
& z=\left(\varphi_{2}\left(-\sigma_{0}\right), \ldots, \varphi_{m}\left(-\sigma_{0}\right)\right), \quad-1-\sigma_{0} \leqslant t \leqslant-\sigma_{0} .
\end{aligned}
$$

Здесь $\mathrm{g}(t, \varepsilon)=\left(\mathrm{g}_{1}(t, \varepsilon), \ldots, \mathrm{g}_{m}(t, \varepsilon)\right), \mathrm{g}(t, \varepsilon)=\mathrm{g}_{0}(t)$ при $-1-\sigma_{0} \leqslant t \leqslant-\sigma_{0},-$ решение линейной системы, получающейся из (3.4), (3.5) при линеаризации на решении (3.9), а функционал $l: \mathscr{F}_{0} \rightarrow \mathbb{R}$ определен аналогичным (2.54) соотношением

$$
l\left(\mathrm{~g}_{0}\right)=\frac{\mathrm{g}_{1}\left(T_{\varphi}-\sigma_{0}, \varepsilon\right)}{\dot{x}_{\varphi}\left(T_{\varphi}-\sigma_{0}, \varepsilon\right)} .
$$

Естественно возникающий вопрос о связи между операторами (3.10) и (3.11) решается следующей теоремой.

ТЕОРема 3.1 (о $C^{1}$-сходимости). Пусть выполнено условие (2.61) и множество $S$ выбрано описанным выше способом. Тогда найдется такое достаточно малое $\varepsilon_{0}=\varepsilon_{0}(S)>0$, что при всех $0<\varepsilon \leqslant \varepsilon_{0}$ оператор $\Pi_{\varepsilon}$ определен на $S$ и удовлетворяет предельным равенствам

$$
\begin{array}{r}
\lim _{\varepsilon \rightarrow 0} \sup _{\varphi \in S}\left\|\Pi_{\varepsilon}(\varphi)-\Pi_{0}(\varphi)\right\|_{\mathscr{F}}=0, \\
\lim _{\varepsilon \rightarrow 0} \sup _{\varphi \in S}\left\|\partial_{\varphi} \Pi_{\varepsilon}(\varphi)-\partial_{\varphi} \Pi_{0}(\varphi)\right\|_{\mathscr{F}_{0} \rightarrow \mathscr{F}_{0}}=0 .
\end{array}
$$

Доказательство этой теоремы опустим, поскольку в случае $m=2$ оно приведено в статье [49]. Переход же от двумерного случая к значениям $m>2$ носит чисто технический характер.

Остановимся на одном важном следствии из $C^{1}$-сходимости, касающемся существования и устойчивости периодических решений системы (3.4), (3.5). В связи с этим обратим внимание, что в силу (3.12), (3.13) предельный оператор (3.11) является надстройкой над соответствующим $(m-1)$-мерным отображением

$$
\left.z \mapsto \Phi(z) \stackrel{\text { def }}{=}\left(y_{1}^{0}(t, z), y_{2}^{0}(t, z), \ldots, y_{m-1}^{0}(t, z)\right)\right|_{t=T_{0}-\sigma_{0}}
$$

где $z=\left(\varphi_{2}\left(-\sigma_{0}\right), \ldots, \varphi_{m}\left(-\sigma_{0}\right)\right)$. 
Действительно, любой неподвижной точке $z=z_{*}$ этого отображения соответствует неподвижная точка

$$
\begin{gathered}
\varphi_{*}(t)=\left(\varphi_{1}^{*}(t), \ldots, \varphi_{m}^{*}(t)\right): \varphi_{1}^{*}(t)=x_{0}(t), \quad \varphi_{j}^{*}(t)=y_{j-1}^{0}\left(t+T_{0}, z_{*}\right), \\
j=2, \ldots, m, \quad-1-\sigma_{0} \leqslant t \leqslant-\sigma_{0},
\end{gathered}
$$

оператора $\Pi_{0}$ (при условии, конечно, что $\left.\varphi_{j}^{*}(t) \in S_{j}, j=2, \ldots, m\right)$. Последние же требования не являются ограничениями, поскольку, как уже говорилось выше, множества $S_{j}, j=2, \ldots, m$, из (3.8) можно считать произвольными замкнутыми и ограниченными подмножествами пространства $C\left[-1-\sigma_{0},-\sigma_{0}\right]$.

Верно и обратное утверждение: если $\varphi_{*}(t)=\left(\varphi_{1}^{*}(t), \ldots, \varphi_{m}^{*}(t)\right) \in S$ есть неподвижная точка оператора $\Pi_{0}$, то с необходимостью $\varphi_{1}^{*}(t)=x_{0}(t)$, а вектор $z_{*}=\left(\varphi_{2}^{*}\left(-\sigma_{0}\right), \ldots, \varphi_{m}^{*}\left(-\sigma_{0}\right)\right)$ таков, что $\Phi\left(z_{*}\right)=z_{*}$. Кроме того, в силу $(3.19)$ спектр линейного оператора $\partial_{\varphi} \Pi_{0}\left(\varphi_{*}\right)$ состоит из двух множеств: собственного значения $\mu=0$ бесконечной кратности и совокупности собственных значений матрицы Якоби $\Phi^{\prime}\left(z_{*}\right)$.

Суммируя изложенные факты, приходим к выводу, что справедлив следующий результат.

ТЕОРема 3.2 (о соответствии). Каждой неподвижной точке $z=z_{*}$ отображения (3.22), удовлетворяющей условию $\operatorname{det}\left(I-\Phi^{\prime}\left(z_{*}\right)\right) \neq 0$, где $I$ - единичная матрица, соответствует релаксационныи цикл системы (3.4), (3.5). Этот иикл существует при всех достаточно малых $\varepsilon>0$ и является экспоненииалъно орбитально устойчивым (неустойчивым) при $r_{*}<1(>1)$, где $r_{*}-$ спектральный радиус матрицъ $\Phi^{\prime}\left(z_{*}\right)$.

ДокАзАтЕльство. Обратимся сначала к вопросу о существовании релаксационного цикла. В связи с этим обозначим через $\varphi_{*}(t) \in S$ неподвижную точку оператора (3.11), отвечающую неподвижной точке $z=z_{*}$ отображения (3.22). Рассмотрим, далее, уравнение

$$
\Pi_{\varepsilon}(\varphi)-\varphi=0
$$

и заметим, что в силу равенств (3.18)-(3.21) и отмеченных выше спектральных свойств оператора $\partial_{\varphi} \Pi_{0}\left(\varphi_{*}\right)$ к данному уравнению в точке $(\varphi, \varepsilon)=\left(\varphi_{*}(t), 0\right)$ пространства $\mathscr{F} \times \mathbb{R}$ применима теорема о неявном отображении по переменной $\varphi$. Таким образом, из (3.23) однозначно определяется неподвижная точка

$$
\varphi=\varphi_{*}^{\varepsilon}(t) \in S, \quad \lim _{\varepsilon \rightarrow 0}\left\|\varphi_{*}^{\varepsilon}(t)-\varphi_{*}(t)\right\|_{\mathscr{F}}=0,
$$

оператора (3.10), а отвечающее ей решение

$$
\left(x(t, \varepsilon), y_{1}(t, \varepsilon), y_{2}(t, \varepsilon), \ldots, y_{m-1}(t, \varepsilon)\right)
$$

системы (3.4), (3.5) будет, очевидно, периодическим с периодом $T(\varepsilon)=\left.T_{\varphi}\right|_{\varphi=\varphi_{*}^{\varepsilon}}$.

Перейдем теперь к вопросу об устойчивости найденного периодического решения (3.25). Из проделанных выше построений следует, что его устойчивость определяется по спектру оператора $\partial_{\varphi} \Pi_{\varepsilon}\left(\varphi_{*}^{\varepsilon}\right)$. Последний же в силу вытекающего из (3.21), (3.24) равенства

$$
\lim _{\varepsilon \rightarrow 0}\left\|\partial_{\varphi} \Pi_{\varepsilon}\left(\varphi_{*}^{\varepsilon}\right)-\partial_{\varphi} \Pi_{0}\left(\varphi_{*}\right)\right\|_{\mathscr{F}_{0} \rightarrow \mathscr{F}_{0}}=0
$$


имеет набор из $m-1$ собственных значений, асимптотически близких к собственным значениям матрицы $\Phi^{\prime}\left(z_{*}\right)$, а остальной его спектр лежит в круге $\left\{\mu \in \mathbb{C}:|\mu| \leqslant r_{0}\right\}$ асимптотически малого по $\varepsilon$ радиуса $r_{0}=r_{0}(\varepsilon)$. Таким образом, свойства устойчивости рассматриваемого цикла совпадают с аналогичными свойствами неподвижной точки $z=z_{*}$ отображения (3.22). Теорема 3.2 доказана.

В дополнение к установленной теореме остановимся на асимптотических свойствах компонент цикла (3.25). Из содержащегося в [49] обоснования теоремы 3.1 вытекают предельные равенства

$$
\begin{gathered}
\lim _{\varepsilon \rightarrow 0} T(\varepsilon)=T_{0}, \quad \lim _{\varepsilon \rightarrow 0} \max _{-\sigma_{0} \leqslant t \leqslant T(\varepsilon)-\sigma_{0}}\left|x(t, \varepsilon)-x_{0}(t)\right|=0, \\
\lim _{\varepsilon \rightarrow 0} \max _{t \in \Sigma(\varepsilon)}\left|y_{j}(t, \varepsilon)-y_{j}^{0}\left(t, z_{*}\right)\right|=0, \quad j=1, \ldots, m-1,
\end{gathered}
$$

где множество $\Sigma(\varepsilon)$ - отрезок $-\sigma_{0} \leqslant t \leqslant T(\varepsilon)-\sigma_{0}$ с выброшенными интервалами вида $\left(1-\varepsilon^{\delta}, 1+\varepsilon^{\delta}\right),\left(t_{0}+1-\varepsilon^{\delta}, t_{0}+1+\varepsilon^{\delta}\right), \delta=$ const $\in(0,1)$.

Теорема 3.2 сводит интересующую нас проблему автоволновых процессов системы (3.1) к поиску неподвижных точек отображения (3.22). Вопрос о количестве и устойчивости последних будет изучен в следующем пункте. Здесь же приведем указанное отображение к некоторому инвариантному виду, не зависящему от начального момента времени $t=-\sigma_{0}$.

Заметим в первую очередь, что интересующее нас отображение представляет собой оператор сдвига по траекториям системы (3.12) с $T_{0}$-периодическим импульсным воздействием за отрезок времени $-\sigma_{0} \leqslant t \leqslant T_{0}-\sigma_{0}$. А это означает, что его можно записать в инвариантной форме, заменяя указанный выше отрезок не зависящим от $\sigma_{0}$ промежутком $0 \leqslant t \leqslant T_{0}$.

Действительно, введем в рассмотрение оператор сдвига $P^{t}(z), P^{0}(z)=z$, $P^{t}(0) \equiv 0$, по траекториям системы (3.14) и положим $\bar{z}=\Phi(z)$. Тогда отображение (3.22) может быть представлено в виде

$$
\bar{z}=\left(P^{T_{0}-t_{0}-1-\sigma_{0}} \circ P_{2} \circ P^{t_{0}} \circ P_{1} \circ P^{1+\sigma_{0}}\right)(z),
$$

где через $P_{1}, P_{2}$ обозначены операторы пересчета начальных условий в точках $t=1$ и $t=t_{0}+1$ соответственно, действующие по правилам

$$
P_{1}(z)=z-(1+a) P^{-1}(z), \quad P_{2}(z)=z-\left(1+\frac{1}{a}\right) P^{-1}(z) .
$$

Далее, применим к левой и правой части получившегося равенства оператор $P^{\sigma_{0}}$. В результате с учетом очевидных соотношений

$$
P^{1+\sigma_{0}}=P^{1} \circ P^{\sigma_{0}}, \quad P^{T_{0}-t_{0}-1-\sigma_{0}}=P^{-\sigma_{0}} \circ P^{T_{0}-t_{0}-1}
$$

имеем

$$
P^{\sigma_{0}}(\bar{z})=\left(P^{T_{0}-t_{0}-1} \circ P_{2} \circ P^{t_{0}} \circ P_{1} \circ P^{1}\right)\left(P^{\sigma_{0}}(z)\right) .
$$

А отсюда, в свою очередь, следует, что после замены $P^{\sigma_{0}}(z) \rightarrow z$ интересующее нас отображение принимает требуемую инвариантную форму

$$
z \rightarrow \Phi_{0}(z) \stackrel{\text { def }}{=}\left(P^{T_{0}-t_{0}-1} \circ P_{2} \circ P^{t_{0}} \circ P_{1} \circ P^{1}\right)(z)
$$


или, что то же самое,

$$
\left.z \rightarrow \Phi_{0}(z) \stackrel{\text { def }}{=}\left(y_{1}^{0}(t, z), y_{2}^{0}(t, z), \ldots, y_{m-1}^{0}(t, z)\right)\right|_{t=T_{0}},
$$

где $\left(y_{1}^{0}(t, z), y_{2}^{0}(t, z), \ldots, y_{m-1}^{0}(t, z)\right)$ - решение аналогичной $(3.12),(3.13)$ задачи Коши для системы (3.12) с начальным условием

$$
\left.\left(y_{1}, \ldots, y_{m-1}\right)\right|_{t=0}=z, \quad z=\left(z_{1}, \ldots, z_{m-1}\right) .
$$

Подчеркнем, что в силу леммы 3.1 это отображение заведомо определено во всем пространстве $\mathbb{R}^{m-1}$.

3.2. Аттракторы предельного отображения. В данном пункте обратимся к актуальному в связи с теоремой 3.2 вопросу о количестве и устойчивости неподвижных точек отображения (3.27). Поиск аттракторов этого отображения начнем с анализа свойств устойчивости его неподвижной точки $z=0$.

ЛЕмма 3.2. Нулевая неподвижная точка отображения (3.27) экспоненииально устойчива при любом $d>0$.

ДокАзАтЕльство. Несложный подсчет показывает, что отвечающая точке $z=0$ матрица Якоби $\Phi_{0}^{\prime}(0)$ есть оператор сдвига по решениям импульсной системы

$$
\begin{gathered}
\dot{\mathrm{h}}_{j}=d\left(\mathrm{~h}_{j+1}-2 \mathrm{~h}_{j}+\mathrm{h}_{j-1}\right), \\
\mathrm{h}_{j}(1+0)=\mathrm{h}_{j}(1-0)-(1+a) \mathrm{h}_{j}(0), \\
\mathrm{h}_{j}\left(t_{0}+1+0\right)=\mathrm{h}_{j}\left(t_{0}+1-0\right)-\left(1+\frac{1}{a}\right) \mathrm{h}_{j}\left(t_{0}\right), \\
j=1, \ldots, m-1, \quad \mathrm{~h}_{0}=\mathrm{h}_{m}=0,
\end{gathered}
$$

за время от $t=0$ до $t=T_{0}$. Далее, применим к (3.29) метод Фурье по собственным векторам разностного оператора Лапласа, а точнее говоря, положим

$$
\mathrm{h}_{j}=\sum_{k=1}^{m-1} \mathrm{~g}_{k}(t) \sin \left(\frac{\pi k}{m} j\right), \quad j=1, \ldots, m-1 .
$$

В результате убеждаемся, что компоненты $\mathrm{g}_{k}(t), k=1, \ldots, m-1$, из (3.30) являются решениями импульсной системы

$$
\begin{gathered}
\dot{\mathrm{g}}=-s \mathrm{~g}, \quad \mathrm{~g}(1+0)=\mathrm{g}(1-0)-(1+a) \mathrm{g}(0), \\
\mathrm{g}\left(t_{0}+1+0\right)=\mathrm{g}\left(t_{0}+1-0\right)-\left(1+\frac{1}{a}\right) \mathrm{g}\left(t_{0}\right)
\end{gathered}
$$

при $0 \leqslant t \leqslant T_{0}, s=s_{k}$, где

$$
s_{k}=4 d \sin ^{2} \frac{\pi k}{2 m}, \quad k=1, \ldots, m-1 .
$$

На завершающем этапе дополним систему (3.31) начальным условием $\mathrm{g}=1$ при $t=0$, проинтегрируем получившуюся задачу Коши на отрезке $0 \leqslant t \leqslant T_{0}$ и положим $\mu_{k}=\left.\mathrm{g}\right|_{t=T_{0}, s=s_{k}}$, где $s_{k}$ - дискретные значения (3.32) параметра $s$. 
В итоге получаем набор чисел $\mu_{k}, k=1, \ldots, m-1$, который, как нетрудно увидеть, образует спектр интересующей нас матрицы $\Phi_{0}^{\prime}(0)$. Более того, справедливы соотношения $\mu_{k}=\left.\mu(s)\right|_{s=s_{k}}, k=1, \ldots, m-1$, где функция $\mu(s)$ задается равенством

$$
\mu(s)=((1+a) \exp s-1)\left(\left(1+\frac{1}{a}\right) \exp s-1\right) \exp \left(-s T_{0}\right)
$$

и обладает свойствами

$$
\begin{aligned}
& \mu(0)= 1, \\
& \mu^{\prime}(s)=\left(\left(2-T_{0}\right) \exp (2 s)+\left(T_{0}-1\right) \exp s-1\right) T_{0} \exp \left(-s T_{0}\right)<0 \\
& \quad \text { для любого } s \in(0,+\infty), \\
& \mu(s) \rightarrow 0 \quad \text { при } s \rightarrow+\infty .
\end{aligned}
$$

А отсюда очевидным образом следует, что $\mu_{k} \in(0,1), k=1, \ldots, m-1$. Лемма 3.2 доказана.

В исходной диффузионной цепочке (3.1) неподвижной точке $z=0$ отвечает однородный цикл (3.2). Лемма 3.2 и теорема 3.2 приводят к выводу, что этот цикл является экспоненциально орбитально устойчивым при любом фиксированном значении $d>0$ и при всех достаточно больших $\lambda$.

Отыскание других устойчивых неподвижных точек отображения (3.27) будем проводить в предположении о малости параметра $d$, что позволяет асимптотически проинтегрировать систему (3.12) на отрезке $0 \leqslant t \leqslant T_{0}$. Соответствующий анализ начнем с простейшего случая, когда компоненты начального условия (3.28) заданы равенствами

$$
z_{j}=\frac{1}{a} \ln \frac{1}{d}+v_{j}, \quad v_{j}=\text { const } \in \mathbb{R}, \quad j=1, \ldots, m-1 .
$$

Зафиксируем произвольно компактное множество $\Omega \subset \mathbb{R}^{m-1}$ и обозначим через

$$
\left(y_{1}(t, v, d), \ldots, y_{m-1}(t, v, d)\right), \quad 0 \leqslant t \leqslant T_{0}, \quad v=\left(v_{1}, \ldots, v_{m-1}\right) \in \Omega,
$$

решение задачи Коши (3.12), (3.28), (3.35). Ниже проводится его асимптотический анализ при $d \rightarrow 0$.

Обратимся сначала к полуинтервалу $0 \leqslant t<1$, на котором для определения фигурирующих в (3.36) функций $y_{j}(t, v, d)$ получается задача Коши

$$
\begin{aligned}
\dot{y}_{j} & =d\left[\exp y_{j+1}+\exp \left(-y_{j}\right)-\exp y_{j}-\exp \left(-y_{j-1}\right)\right], \\
\left.y_{j}\right|_{t=0} & =\frac{1}{a} \ln \frac{1}{d}+v_{j}, \quad j=1, \ldots, m-1, \quad y_{0}=y_{m}=0 .
\end{aligned}
$$

Ее решение будем искать в виде

$$
y_{j}(t, v, d)=\frac{1}{a} \ln \frac{1}{d}+\omega_{j}(t, v, d), \quad \omega_{j}(0, v, d)=v_{j}, \quad j=1, \ldots, m-1 .
$$


Подставляя затем соотношения (3.38) в (3.37), приходим к выводу, что равномерно по $t \in[0,1), v \in \Omega$

$$
\begin{gathered}
\omega_{j}(t, v, d)=v_{j}+O\left(d^{1-1 / a}\right), \quad \frac{\partial \omega_{j}}{\partial v_{k}}(t, v, d)=\delta_{j k}+O\left(d^{1-1 / a}\right), \quad d \rightarrow 0, \\
j, k=1, \ldots, m-1,
\end{gathered}
$$

где $\delta_{j k}-$ символ Кронекера.

Далее, в момент времени $t=1$ первый раз происходит скачок и согласно $(3.12),(3.38),(3.39)$ при $t \in\left[1, t_{0}+1\right)$ имеем дело с задачей Коши

$$
\begin{gathered}
\dot{y}_{j}=d\left[\exp y_{j+1}+\exp \left(-y_{j}\right)-\exp y_{j}-\exp \left(-y_{j-1}\right)\right], \\
\left.y_{j}\right|_{t=1}=-\ln \frac{1}{d}-a v_{j}+O\left(d^{1-1 / a}\right), \quad j=1, \ldots, m-1, \quad y_{0}=y_{m}=0 .
\end{gathered}
$$

Как и выше, ее решение ищем в аналогичном (3.38) виде

$$
y_{j}(t, v, d)=-\ln \frac{1}{d}+\omega_{j}(t, v, d), \quad j=1, \ldots, m-1 .
$$

В итоге с точностью до величин порядка $d^{1-1 / a}$ для функций $\omega_{j}(t, v, d)$ из $(3.41)$ приходим к задаче Коши

$$
\begin{gathered}
\dot{\omega}_{1}=\exp \left(-\omega_{1}\right), \quad \dot{\omega}_{j}=\exp \left(-\omega_{j}\right)-\exp \left(-\omega_{j-1}\right), \quad j=2, \ldots, m-1, \\
\left.\omega_{j}\right|_{t=1}=-a v_{j}, \quad j=1, \ldots, m-1
\end{gathered}
$$

решение которой обозначим через $\left(\omega_{1}^{0}(t, v), \ldots, \omega_{m-1}^{0}(t, v)\right)$. Что же касается исходной задачи (3.40), то ее решение допускает в данном случае равномерные по $t \in\left[1, t_{0}+1\right), v \in \Omega$ асимптотические представления

$$
\begin{gathered}
y_{j}(t, v, d)=-\ln \frac{1}{d}+\omega_{j}^{0}(t, v)+O\left(d^{1-1 / a}\right), \\
\frac{\partial y_{j}}{\partial v_{k}}(t, v, d)=\frac{\partial \omega_{j}^{0}}{\partial v_{k}}(t, v)+O\left(d^{1-1 / a}\right), \\
j, k=1, \ldots, m-1, \quad d \rightarrow 0 .
\end{gathered}
$$

При $t=t_{0}+1$ происходит очередной скачок, и в силу (3.43), (3.12) на оставшемся отрезке $t_{0}+1 \leqslant t \leqslant T_{0}$ получается задача Коши

$$
\begin{gathered}
\dot{y}_{j}=d\left[\exp y_{j+1}+\exp \left(-y_{j}\right)-\exp y_{j}-\exp \left(-y_{j-1}\right)\right], \\
\left.y_{j}\right|_{t=0}=\frac{1}{a} \ln \frac{1}{d}+\psi_{j}(v)+O\left(d^{1-1 / a}\right), \\
j=1, \ldots, m-1, \quad y_{0}=y_{m}=0
\end{gathered}
$$

где

$$
\psi_{j}(v)=\left.\omega_{j}^{0}(t, v)\right|_{t=t_{0}+1}-\left.\left(1+\frac{1}{a}\right) \omega_{j}^{0}(t, v)\right|_{t=t_{0}}, \quad j=1, \ldots, m-1 .
$$


Опуская ее несложный анализ, приведем сразу вытекающие из (3.44), (3.45) окончательные формулы

$$
\begin{gathered}
y_{j}(t, v, d)=\frac{1}{a} \ln \frac{1}{d}+\psi_{j}(v)+O\left(d^{1-1 / a}\right), \\
\frac{\partial y_{j}}{\partial v_{k}}(t, v, d)=\frac{\partial \psi_{j}}{\partial v_{k}}(v)+O\left(d^{1-1 / a}\right), \\
j, k=1, \ldots, m-1, \quad d \rightarrow 0,
\end{gathered}
$$

равномерные по $t \in\left[t_{0}+1, T_{0}\right], v \in \Omega$.

Проделанные построения показывают, что после перехода к новым переменным $v_{j}, j=1, \ldots, m-1$, интересующее нас отображение (3.27) записывается с помощью равенств (3.35) в виде

$$
v_{j} \rightarrow \psi_{j}(v)+\Delta_{j}(v, d), \quad j=1, \ldots, m-1,
$$

где остатки $\Delta_{j}(v, d)$ при $d \rightarrow 0$ имеют порядок малости $O\left(d^{1-1 / a}\right)$ в метрике пространства $C^{1}(\Omega)$. А это значит, что при изучении вопроса о неподвижных точках мы вправе заменить (3.47) предельным отображением

$$
v_{j} \rightarrow \psi_{j}(v), \quad j=1, \ldots, m-1 .
$$

Исследование отображения (3.48) существенно опирается на тот факт, что компоненты $\omega_{j}^{0}(t, v)$ решения задачи $(3.42)$ могут быть найдены в явном виде. Действительно, непосредственная проверка показывает, что для них справедливы равенства

$$
\begin{gathered}
\omega_{1}^{0}(t, v)+\cdots+\omega_{k}^{0}(t, v)=\ln \left\{\frac{(t-1)^{k}}{k !}+\sum_{\ell=0}^{k-1} \frac{(t-1)^{\ell}}{\ell !} \exp \left(-a \sum_{j=1}^{k-\ell} v_{j}\right)\right\}, \\
k=1, \ldots, m-1 .
\end{gathered}
$$

Далее, подставим (3.49) в (3.45) и перейдем к новым переменным

$$
\alpha_{k}=v_{1}+\cdots+v_{k}, \quad k=1, \ldots, m-1 .
$$

В результате наше отображение примет треугольный вид

$$
\begin{gathered}
\alpha_{k} \rightarrow \ln \left(r_{1, k}+\exp \left(-a \alpha_{k}\right)\right)-\left(1+\frac{1}{a}\right) \ln \left(r_{2, k}+\exp \left(-a \alpha_{k}\right)\right), \\
k=1, \ldots, m-1,
\end{gathered}
$$

где

$$
\begin{gathered}
r_{1,1}=1+\frac{1}{a}, \quad r_{2,1}=\frac{1}{a} \\
r_{1, k}\left(\alpha_{1}, \ldots, \alpha_{k-1}\right)=\frac{(1+1 / a)^{k}}{k !}+\sum_{\ell=1}^{k-1} \frac{(1+1 / a)^{\ell}}{\ell !} \exp \left(-a \alpha_{k-\ell}\right), \\
r_{2, k}\left(\alpha_{1}, \ldots, \alpha_{k-1}\right)=\frac{1}{a^{k} k !}+\sum_{\ell=1}^{k-1} \frac{1}{a^{\ell} \ell !} \exp \left(-a \alpha_{k-\ell}\right), \quad k=2, \ldots, m-1 .
\end{gathered}
$$


Анализ получившегося отображения тесно связан со свойствами вспомогательного одномерного отображения

$$
\alpha \rightarrow \psi_{r_{1}, r_{2}}(\alpha) \stackrel{\text { def }}{=} \ln \left(r_{1}+\exp (-a \alpha)\right)-\left(1+\frac{1}{a}\right) \ln \left(r_{2}+\exp (-a \alpha)\right)
$$

с двумя параметрами $r_{1}, r_{2}>0$, удовлетворяющими условию

$$
r_{1}>\left(1+\frac{1}{a}\right) r_{2}
$$

Справедливо следующее утверждение.

ЛЕмма 3.3. При выполнении неравенства (3.55) отображсние (3.54) имеет единственную глобально экспоненциально устойчивую неподвижную точ$\kappa y \alpha_{*}=\alpha_{*}\left(r_{1}, r_{2}\right)$.

ДокАзАТЕЛЬство. Отметим сначала вытекающие из явного вида функции $\psi_{r_{1}, r_{2}}(\alpha)($ см. (3.54)) асимптотические свойства

$$
\begin{gathered}
\lim _{\alpha \rightarrow+\infty} \psi_{r_{1}, r_{2}}(\alpha)=\ln r_{1}-\left(1+\frac{1}{a}\right) \ln r_{2}, \\
\psi_{r_{1}, r_{2}}(\alpha)=\alpha+\left(r_{1}-\left(1+\frac{1}{a}\right) r_{2}\right) \exp (a \alpha)+O(\exp (2 a \alpha)), \quad \alpha \rightarrow-\infty .
\end{gathered}
$$

Учитывая, далее, в (3.56) условие (3.55), приходим к выводу, что при всех достаточно больших $\alpha>0$ выполняется неравенство $\psi_{r_{1}, r_{2}}(\alpha)<\alpha$, а при всех достаточно больших по модулю отрицательных $\alpha$ - неравенство $\psi_{r_{1}, r_{2}}(\alpha)>\alpha$. Таким образом, отображение (3.54) заведомо имеет хотя бы одну неподвижную точку $\alpha=\alpha_{*}$, являющуюся, очевидно, корнем уравнения

$$
\exp \alpha_{*}=\frac{r_{1}+\exp \left(-a \alpha_{*}\right)}{\left(r_{2}+\exp \left(-a \alpha_{*}\right)\right)^{1+1 / a}} .
$$

Перейдем теперь к вопросу об устойчивости найденной неподвижной точки. В связи с этим обратим внимание на то, что требование (3.55) влечет за собой выполнение неравенства $r_{1}>r_{2}$. Используя данный факт, убеждаемся, что, во-первых,

$$
\begin{aligned}
& \frac{d}{d \alpha} \psi_{r_{1}, r_{2}}(\alpha)=\exp (-a \alpha) \\
& \quad \times\left(\frac{a\left(r_{1}-r_{2}\right)}{\left(r_{1}+\exp (-a \alpha)\right)\left(r_{2}+\exp (-a \alpha)\right)}+\frac{1}{r_{2}+\exp (-a \alpha)}\right)>0 \\
& \forall \alpha \in \mathbb{R}
\end{aligned}
$$

и, во-вторых, за устойчивость неподвижной точки $\alpha=\alpha_{*}$ отвечает мультипликатор

$$
\left.\mu_{*} \stackrel{\text { def }}{=} \frac{d}{d \alpha} \psi_{r_{1}, r_{2}}(\alpha)\right|_{\alpha=\alpha_{*}}=\left.\left(\frac{v_{*}+r_{2}}{v_{*}+r_{1}}\right)^{a}\left(1+\frac{a\left(r_{1}-r_{2}\right)}{v_{*}+r_{1}}\right)\right|_{v_{*}=\exp \left(-a \alpha_{*}\right)}
$$


являющийся положительным (при выводе равенства (3.59) привлекались соотношения $(3.57),(3.58))$. Кроме того, учитывая, что $a>1$, и опираясь на очевидные оценки

$$
\left(\frac{v_{*}+r_{1}}{v_{*}+r_{2}}\right)^{a}=\left(1+\frac{r_{1}-r_{2}}{v_{*}+r_{2}}\right)^{a}>1+\frac{a\left(r_{1}-r_{2}\right)}{v_{*}+r_{2}}>1+\frac{a\left(r_{1}-r_{2}\right)}{v_{*}+r_{1}},
$$

нетрудно заметить, что $\mu_{*}<1$.

Итак, мы убедились, что любая возможная неподвижная точка $\alpha=\alpha_{*}$ отображения (3.54) является экспоненциально устойчивой. А это значит, что на самом деле такая точка единственна и в силу монотонности нашего отображения (см. (3.58)) глобально устойчива. Лемма 3.3 доказана.

Вернемся к отображению (3.51) и заметим, что от него очевидным образом "отщепляется" одномерное отображение для координаты $\alpha_{1}$, имеющее вид

$$
\left.\alpha_{1} \rightarrow \psi_{r_{1}, r_{2}}\left(\alpha_{1}\right)\right|_{r_{1}=r_{1,1}, r_{2}=r_{2,1}}
$$

где $\psi_{r_{1}, r_{2}}$ - отображение (3.54). Кроме того, параметры $r_{1,1}, r_{2,1}$, задаваемые равенствами (3.52), удовлетворяют условию (3.55) (это условие эквивалентно требованию (2.61), которое мы а priori считаем выполненным). А отсюда и из леммы 3.3 вытекает существование у (3.60) единственной экспоненциально устойчивой неподвижной точки $\alpha_{1}=\alpha_{1}^{*}$.

Предположим теперь, что уже найдены первые $k-1$ координат $\alpha_{1}^{*}, \ldots, \alpha_{k-1}^{*}$ устойчивой неподвижной точки отображения (3.51). Тогда после подстановки в (3.53) известных значений $\alpha_{s}=\alpha_{s}^{*}, s=1, \ldots, k-1$, для определения $\alpha_{k}$ получаем аналогичное (3.60) одномерное отображение

$$
\left.\alpha_{k} \rightarrow \psi_{r_{1}, r_{2}}\left(\alpha_{k}\right)\right|_{r_{1}=r_{1, k}^{*}, r_{2}=r_{2, k}^{*}}
$$

где $r_{1, k}^{*}=r_{1, k}\left(\alpha_{1}^{*}, \ldots, \alpha_{k-1}^{*}\right), r_{2, k}^{*}=r_{2, k}\left(\alpha_{1}^{*}, \ldots, \alpha_{k-1}^{*}\right)$. Заметим, далее, что и здесь условие (3.55) остается в силе, поскольку

$$
\begin{aligned}
r_{1, k}^{*} & -\left(1+\frac{1}{a}\right) r_{2, k}^{*}=\left(1+\frac{1}{a}\right)\left\{\frac{1}{k !}\left(\left(1+\frac{1}{a}\right)^{k-1}-\frac{1}{a^{k}}\right)\right. \\
& \left.+\sum_{\ell=1}^{k-1} \frac{1}{\ell !}\left(\left(1+\frac{1}{a}\right)^{\ell-1}-\frac{1}{a^{\ell}}\right) \exp \left(-a \alpha_{k-\ell}^{*}\right)\right\}
\end{aligned}
$$

и $(1+1 / a)^{\ell-1}>1 / a^{\ell}$ для всех $\ell \leqslant k$. А это означает, что отображение (3.61) имеет единственную экспоненциально устойчивую неподвижную точку $\alpha_{k}=\alpha_{k}^{*}$.

Продолжая описанный процесс вплоть до номера $k=m-1$, убеждаемся, что исходное отображение (3.51) допускает единственную экспоненциально устойчивую неподвижную точку с компонентами $\left(\alpha_{1}^{*}, \ldots, \alpha_{m-1}^{*}\right)$. Далее, обращая замену (3.50), получаем устойчивую неподвижную точку $\left(v_{1}^{*}, \ldots, v_{m-1}^{*}\right)$ отображения (3.48) и соответствующую близкую к ней неподвижную точку $\left(v_{1}(d), \ldots, v_{m-1}(d)\right): v_{j}(0)=v_{j}^{*}, j=1, \ldots, m-1$, отображения (3.47). Что же 
касается отображения (3.27), то координаты его устойчивой неподвижной точки задаются равенствами

$$
z_{j}=\frac{1}{a} \ln \frac{1}{d}+v_{j}(d), \quad j=1, \ldots, m-1 .
$$

Следует добавить, что неподвижная точка (3.62) существует в паре с устойчивой неподвижной точкой

$$
z_{j}=-\frac{1}{a} \ln \frac{1}{d}-v_{m-j}(d), \quad j=1, \ldots, m-1 .
$$

Наличие последней обусловлено тем обстоятельством, что отображение (3.27) инвариантно по отношению к замене $z_{j} \rightarrow-z_{m-j}, j=1, \ldots, m-1$. Отметим еще, что неподвижным точкам (3.62), (3.63) в диффузионной цепочке (3.1) отвечает пара устойчивых релаксационных циклов, переходящих друг в друга при замене $u_{j} \rightarrow u_{m+1-j}, j=1, \ldots, m$.

Завершая обсуждение свойств отображения (3.27), отметим, что помимо найденных выше трех устойчивых неподвижных точек у него имеются и другие аттракторы. Для отыскания части из них зафиксируем натуральное $k_{0}$, $k_{0} \leqslant m-2$, и дополним систему (3.12) аналогичными (3.35) начальными условиями

$$
\begin{array}{rlrl}
\left.y_{j}\right|_{t=0}=-\frac{1}{a} \ln \frac{1}{d}+v_{j}, & j & j, \ldots, k_{0} ; \\
\left.y_{j}\right|_{t=0}=\frac{1}{a} \ln \frac{1}{d}+v_{j}, & j=k_{0}+1, \ldots, m-1,
\end{array}
$$

где по-прежнему параметр $d$ мал, $v_{j}=$ const $\in \mathbb{R}, j=1, \ldots, m-1$, а постоянная $а$ удовлетворяет оценке (2.61). В результате для компонент $y_{j}(t, v, d)$, $j=1, \ldots, m-1$, решения получившейся задачи Коши (3.12), (3.64) приходим к аналогичным (3.38)-(3.46) асимптотическим равенствам:

$$
\begin{array}{ll}
y_{j}=-\frac{1}{a} \ln \frac{1}{d}+v_{j}+O\left(d^{1-1 / a}\right), & j=1, \ldots, k_{0}, \\
y_{j}=\frac{1}{a} \ln \frac{1}{d}+v_{j}+O\left(d^{1-1 / a}\right), & j=k_{0}+1, \ldots, m-1,
\end{array}
$$

при $t \in[0,1)$;

$$
\begin{array}{ll}
y_{j}=\ln \frac{1}{d}+\omega_{j}^{0}(t, v)+O\left(d^{1-1 / a}\right), & j=1, \ldots, k_{0}, \\
y_{j}=-\ln \frac{1}{d}+\omega_{j}^{0}(t, v)+O\left(d^{1-1 / a}\right), & j=k_{0}+1, \ldots, m-1
\end{array}
$$

при $t \in\left[1, t_{0}+1\right)$;

$$
\begin{array}{ll}
y_{j}=-\frac{1}{a} \ln \frac{1}{d}+\psi_{j}(v)+O\left(d^{1-1 / a}\right), & j=1, \ldots, k_{0}, \\
y_{j}=\frac{1}{a} \ln \frac{1}{d}+\psi_{j}(v)+O\left(d^{1-1 / a}\right), & j=k_{0}+1, \ldots, m-1,
\end{array}
$$

при $t \in\left[t_{0}+1, T_{0}\right]$, где $\left(\omega_{1}^{0}(t, v), \ldots, \omega_{m-1}^{0}(t, v)\right)$ - решение задачи Коши

$$
\begin{gathered}
\dot{\omega}_{j}=\exp \omega_{j+1}-\exp \omega_{j}, \quad j=1, \ldots, k_{0}-1, \quad \dot{\omega}_{k_{0}}=-\exp \omega_{k_{0}}, \\
\left.\omega_{j}\right|_{t=1}=-a v_{j}, \quad j=1, \ldots, k_{0} ;
\end{gathered}
$$




$$
\begin{gathered}
\dot{\omega}_{k_{0}+1}=\exp \left(-\omega_{k_{0}+1}\right) \\
\dot{\omega}_{j}=\exp \left(-\omega_{j}\right)-\exp \left(-\omega_{j-1}\right), \quad j=k_{0}+2, \ldots, m-1, \\
\left.\omega_{j}\right|_{t=1}=-a v_{j}, \quad j=k_{0}+1, \ldots, m-1,
\end{gathered}
$$

а для функций $\psi_{j}(v), j=1, \ldots, m-1$, сохраняются формулы (3.45).

Дальнейший способ действий аналогичен изложенному выше: после подстановки в (3.27) соотношений

$$
\begin{array}{rlrl}
z_{j} & =-\frac{1}{a} \ln \frac{1}{d}+v_{j}, & & j=1, \ldots, k_{0}, \\
z_{j}=\frac{1}{a} \ln \frac{1}{d}+v_{j}, & j=k_{0}+1, \ldots, m-1,
\end{array}
$$

и предельного перехода при $d \rightarrow 0$ (законность которого в метрике пространства $C^{1}(\Omega)$ вытекает из формул (3.65)-(3.67)) получаем соответствующее отображение для $v=\left(v_{1}, \ldots, v_{m-1}\right)$. Специфика рассматриваемого случая заключается в том, что в силу независимости подсистем (3.68) и (3.69) упомянутое отображение также распадается на два независимых отображения

$$
\begin{array}{ll}
v_{j} \rightarrow \psi_{j}\left(v_{1}, \ldots, v_{k_{0}}\right), & j=1, \ldots, k_{0} \\
v_{j} \rightarrow \psi_{j}\left(v_{k_{0}+1}, \ldots, v_{m-1}\right), & j=k_{0}+1, \ldots, m-1 .
\end{array}
$$

Сравнивая системы (3.42) и (3.69), нетрудно заметить, что после надлежащего переобозначения координат отображение (3.71) в точности совпадет с отображением (3.48) (при замене в последнем $m$ на $m-k_{0}$ ). Что же касается отображения (3.70), то оно приводится к (3.48) в результате преобразований $v_{j} \rightarrow-v_{k_{0}+1-j}, j=1, \ldots, k_{0}$. А отсюда и из проделанного выше анализа вытекает, что полное отображение (3.70), (3.71) имеет единственную экспоненциально устойчивую неподвижную точку $\left(v_{1, k_{0}}^{*}, \ldots, v_{m-1, k_{0}}^{*}\right)$.

Суммируя проделанные построения и меняя $k_{0}$ в пределах от единицы до $m-2$, приходим к выводу, что при всех достаточно малых $d>0$ и при условии (2.61) отображение (3.27) допускает набор из $m-2$ устойчивых неподвижных точек с координатами

$$
\begin{aligned}
z_{j}=-\frac{1}{a} \ln \frac{1}{d}+v_{j, k_{0}}^{*}+O\left(d^{1-1 / a}\right), & j=1, \ldots, k_{0} ; \\
z_{j}=\frac{1}{a} \ln \frac{1}{d}+v_{j, k_{0}}^{*}+O\left(d^{1-1 / a}\right), & j=k_{0}+1, \ldots, m-1, \quad d \rightarrow 0,
\end{aligned}
$$

где $v_{j, k_{0}}^{*}, j=1, \ldots, m-1,-$ компоненты неподвижной точки предельного отображения (3.70), (3.71). Общее же количество найденных нами устойчивых ненулевых неподвижных точек отображения (3.27) равно $m$ (см. формулы (3.62), (3.63), (3.72)).

Подведем некоторый итог. Теорема 3.2 о соответствии и проделанный выше асимптотический анализ отображения (3.27) приводят к следующему утверждению.

Теорема 3.3. Пусть параметр а удовлетворяет неравенству (2.61). Тогда для любых достаточно малых $d_{2}>d_{1}>0$ найдется такое достаточно 
большое $\lambda_{0}=\lambda_{0}\left(d_{1}, d_{2}\right)>0$, что при всех $d_{1} \leqslant d \leqslant d_{2}, \lambda \geqslant \lambda_{0}$ система (3.1) имеет не менее $m$ экспоненциально орбитально устойчивых автоволновых периодических движений, сосуществующих с устойчивым ииклом (3.2).

В дополнение к сформулированной теореме отметим, что в силу свойств (3.26) и равенств (3.3) графики компонент $u_{j}, j=1, \ldots, m$, любого устойчивого автоволнового периодического режима по форме близки к профилю $u_{*}(t, \lambda)$ однородного цикла (см. рис. 5), но отличаются друг от друга амплитудами и фазовыми сдвигами. В количественном отношении это отличие характеризуют функции $y_{j}=\ln \left(u_{j+1} / u_{j}\right), j=1, \ldots, m-1$, которые оказываются ограниченными по $t, \lambda$.

Наглядное представление о вышесказанном дает рис. 7, где представлены графики на плоскости $(t, u)$ компонент $u_{1}(t), u_{2}(t)$ одного из устойчивых неоднородных циклов системы (3.1) при $m=2, f(u)=(1-u) /(1+0.5 u), \lambda=7.8$, $d=0.05$ (сплошной линией изображена функция $u_{1}(t)$, а пунктирной $\left.-u_{2}(t)\right)$.

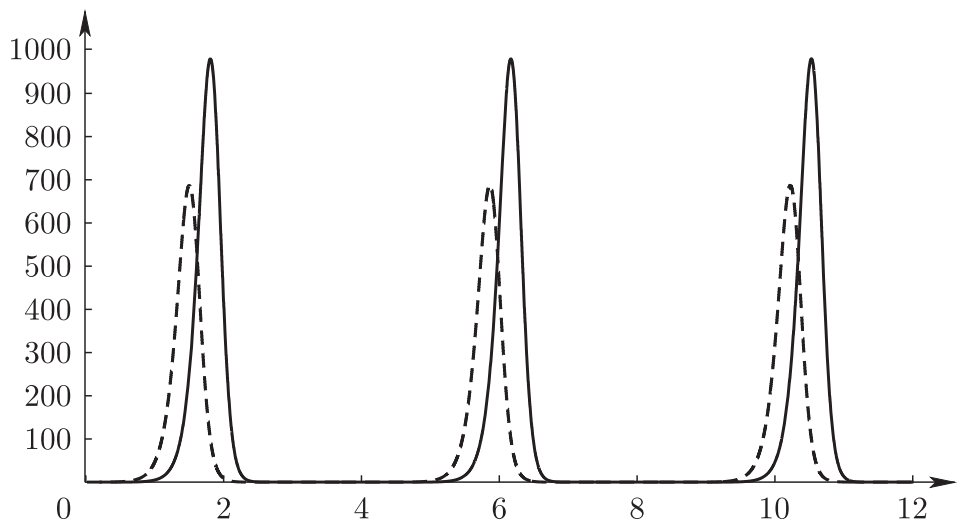

Рис. 7

3.3. Случай кольцевой однонаправленной связи. В данном пункте исследованию подлежит аналогичная (1.37) кольцевая система

$$
\dot{u}_{j}=d\left(u_{j+1}-u_{j}\right)+\lambda f\left(u_{j}(t-1)\right) u_{j}, \quad j=1, \ldots, m, \quad u_{m+1}=u_{1},
$$

где $\lambda \gg 1, d=$ const $>0$, функция $f(u)$ удовлетворяет прежним условиям $(1.49),(1.50),(2.61)$.

Отметим сразу, что для системы (3.73) остаются в силе аналоги базовых теорем 3.1, 3.2. А именно, после замен (3.3) она преобразуется к виду

$$
\begin{gathered}
\dot{x}=\varepsilon d\left(\exp y_{1}-1\right)+F(x(t-1), \varepsilon), \\
\dot{y}_{j}=d\left[\exp y_{j+1}-\exp y_{j}\right]+G_{j}\left(x(t-1), y_{1}(t-1), \ldots, y_{j}(t-1), \varepsilon\right), \\
j=1, \ldots, m-1,
\end{gathered}
$$

где $y_{m}=-y_{1}-y_{2}-\cdots-y_{m-1}$, а функции $F, G_{j}$ те же, что в $(3.4),(3.5)$. Далее, как и выше, проблемы существования и устойчивости периодических 
решений получившейся системы (3.74), (3.75) сводятся к анализу неподвижных точек отображения $(3.27)$, в котором теперь $\left(y_{1}^{0}(t, z), \ldots, y_{m-1}^{0}(t, z)\right)$ - решение импульсной задачи Коши

$$
\begin{gathered}
\dot{y}_{j}=d\left[\exp y_{j+1}-\exp y_{j}\right], \quad y_{j}(1+0)=y_{j}(1-0)-(1+a) y_{j}(0), \\
y_{j}\left(t_{0}+1+0\right)=y_{j}\left(t_{0}+1-0\right)-\left(1+\frac{1}{a}\right) y_{j}\left(t_{0}\right), \quad j=1, \ldots, m-1, \\
y_{m}=-y_{1}-y_{2}-\cdots-y_{m-1}, \\
\left.\left(y_{1}, \ldots, y_{m-1}\right)\right|_{t=0}=z, \quad z=\left(z_{1}, \ldots, z_{m-1}\right) .
\end{gathered}
$$

Проблема корректности определения отображения (3.27) в данном случае связана с системой

$$
\dot{y}_{j}=d\left[\exp y_{j+1}-\exp y_{j}\right], \quad j=1, \ldots, m-1, \quad y_{m}=-y_{1}-y_{2}-\cdots-y_{m-1} .
$$

Убедимся, что для нее сохраняется утверждение леммы 3.1.

Для проверки требуемого факта заметим, что любое решение системы (3.78) записывается в виде $(3.15)$, где теперь $\left(\xi_{1}(t), \ldots, \xi_{m}(t)\right)$ - произвольное решение линейной системы

$$
\dot{\xi}_{j}=d\left(\xi_{j+1}-\xi_{j}\right), \quad j=1, \ldots, m, \quad \xi_{m+1}=\xi_{1},
$$

лежащее в инвариантном конусе $K=\left\{\left(\xi_{1}, \ldots, \xi_{m}\right): \xi_{j}>0, j=1, \ldots, m\right\}$. Заметим, далее, что эта система обладает теми же общими свойствами, что и система (3.16). В частности, для любого ее решения из конуса $K$ имеют место соотношения (3.17). А отсюда автоматически следует стремление к нулю при $t \rightarrow+\infty$ всех решений исходной системы (3.78).

Обратимся теперь непосредственно к анализу отображения (3.27). Ниже в предположении малости $d$ будут найдены две группы его устойчивых неподвижных точек. В случае первой из этих групп мы усилим условие (2.61) и предположим, что

$$
a>m-1 .
$$

Итак, пусть выполнено условие (3.79). Тогда подставим в (3.77) соотношения

$$
z_{j}=-\frac{1}{a} \ln \frac{1}{d}+v_{j}, \quad j=1, \ldots, m-1,
$$

где $v=\left(v_{1}, \ldots, v_{m-1}\right) \in \Omega, \Omega$ - произвольный компакт, и, как обычно, обозначим через $\left(y_{1}(t, v, d), \ldots, y_{m-1}(t, v, d)\right)$ решение получившейся задачи Коши. Несложный ее анализ приводит к выводу, что при $d \rightarrow 0$ справедлива серия асимптотических равенств:

$$
\begin{array}{ll}
y_{j}(t, v, d)=-\frac{1}{a} \ln \frac{1}{d}+v_{j}+O\left(d^{1-(m-1) / a}\right) & \text { при } 0 \leqslant t<1, \\
y_{j}(t, v, d)=\ln \frac{1}{d}+\omega_{j}^{0}(t, v)+O\left(d^{1-(m-1) / a}\right) & \text { при } 1 \leqslant t<t_{0}+1, \\
y_{j}(t, v, d)=-\frac{1}{a} \ln \frac{1}{d}+\psi_{j}(v)+O\left(d^{1-(m-1) / a}\right) & \text { при } t_{0}+1 \leqslant t \leqslant T_{0},
\end{array}
$$


остатки в которых равномерны по $t, v$ и сохраняют свой порядок малости при дифференцировании по $v$. Далее, фигурирующие в $(3.82)$ функции $\omega_{j}^{0}(t, v)$, $j=1, \ldots, m-1$, определяются из задачи Коши

$$
\begin{gathered}
\dot{\omega}_{j}=\exp \omega_{j+1}-\exp \omega_{j}, \quad j=1, \ldots, m-2, \quad \dot{\omega}_{m-1}=-\exp \omega_{m-1}, \\
\left.\omega_{j}\right|_{t=1}=-a v_{j}, \quad j=1, \ldots, m-1,
\end{gathered}
$$

и имеют вид

$$
\begin{aligned}
& \omega_{m-1}^{0}(t, v)+\cdots+\omega_{m-s}^{0}(t, v) \\
& \quad=-\ln \left\{\frac{(t-1)^{s}}{s !}+\sum_{\ell=0}^{s-1} \frac{(t-1)^{\ell}}{\ell !} \exp \left(a \sum_{j=1}^{s-\ell} v_{m-j}\right)\right\},
\end{aligned}
$$

$s=1, \ldots, m-1$. Что же касается функций $\psi_{j}(v)$ из $(3.83)$, то они задаются равенствами вида (3.45).

Перечисленные факты (3.81)-(3.84) свидетельствуют о том, что после замены переменных (3.80) отображение (3.27) имеет при $d \rightarrow 0$ предел вида (3.48) (в метрике $\left.C^{1}(\Omega)\right)$. В свою очередь, отображение (3.48) после перехода к переменным $\alpha_{s}=-v_{m-1}-\cdots-v_{m-s}, s=1, \ldots, m-1$, принимает в точности вид (3.51)-(3.53). Таким образом, мы можем утверждать, что оно имеет единственную экспоненциально устойчивую неподвижную точку $\left(\alpha_{1}^{*}, \ldots, \alpha_{m-1}^{*}\right)$, которой в исходном отображении (3.27) отвечает устойчивая неподвижная точка вида

$$
\begin{gathered}
z_{*}=\left(z_{1}^{*}, \ldots, z_{m-1}^{*}\right), \quad z_{j}^{*}=-\frac{1}{a} \ln \frac{1}{d}+v_{j}^{*}+O\left(d^{1-(m-1) / a}\right), \\
j=1, \ldots, m-1, \quad d \rightarrow 0,
\end{gathered}
$$

где $v_{m-1}^{*}=-\alpha_{1}^{*}, v_{m-s}^{*}=\alpha_{s-1}^{*}-\alpha_{s}^{*}, s=2, \ldots, m-1$.

Интересно отметить, что в случае кольцевой системы отображение (3.27) инвариантно относительно преобразования

$$
\left(z_{1}, \ldots, z_{m-1}\right) \stackrel{\mathscr{A}}{\rightarrow}\left(z_{2}, z_{3}, \ldots, z_{m-1},-z_{1}-z_{2}-\cdots-z_{m-1}\right) .
$$

Поэтому найденная нами неподвижная точка (3.85) порождает сразу $m$ устойчивых неподвижных точек

$$
z_{*}^{(k)}=\mathscr{A}^{k} z_{*}, \quad k=0,1, \ldots, m-1
$$

(подчеркнем, что $\mathscr{A}^{m}=I$, где $I$ - единичный оператор). Остается добавить, что в исходной системе (3.73) этим точкам отвечает набор из $m$ устойчивых релаксационных периодических движений, переходящих друг в друга при циклических перестановках координат $u_{j}, j=1, \ldots, m$.

При отыскании второй группы устойчивых неподвижных точек отображения (3.27) вернемся к прежнему условию (2.61) на параметр $а$. Как и в предыдущем случае, здесь достаточно знать какую-либо одну устойчивую неподвижную точку, а остальные получаются из нее по правилам (3.86), (3.87). 
Итак, положим в (3.77)

$$
z_{j}=\frac{1}{a(m-1)} \ln \frac{1}{d}+v_{j}, \quad j=1, \ldots, m-1, \quad v=\left(v_{1}, \ldots, v_{m-1}\right) \in \Omega,
$$

где, как и ранее, $\Omega$ - произвольный компакт. Характерная особенность данного случая состоит в том, что при условиях (3.88) предельное отображение (3.48) оказывается тождественным по части переменных. Поэтому ниже при построении асимптотики при $d \rightarrow 0$ компонент $y_{j}(t, v, d), j=1, \ldots, m-1$, решения задачи Коши (3.76), (3.77), (3.88) в некоторых формулах будут учтены поправки порядка $d^{\sigma}$, где $\sigma=1-[a(m-1)]^{-1}>0$.

Обратимся сначала к промежутку $0 \leqslant t<1$. Несложный подсчет показывает, что здесь

$$
y_{j}(t, v, d)=\frac{1}{a(m-1)} \ln \frac{1}{d}+v_{j}+d^{\sigma} \delta_{j}(t, v)+o\left(d^{\sigma}\right), \quad j=1, \ldots, m-1,
$$

где

$$
\delta_{j}=t\left(\exp v_{j+1}-\exp v_{j}\right), \quad j=1, \ldots, m-2, \quad \delta_{m-1}=-t \exp v_{m-1} .
$$

Далее, при $1 \leqslant t<t_{0}+1$ с учетом уже установленных соотношений (3.89), (3.90) приходим к равенствам

$$
\begin{aligned}
y_{j}(t, v, d)=- & \frac{1}{m-1} \ln \frac{1}{d}-a v_{j} \\
& +d^{\sigma}\left(\exp v_{j+1}-\exp v_{j}\right)+o\left(d^{\sigma}\right), \quad j=1, \ldots, m-2 ; \\
y_{m-1}(t, v, d)=- & \frac{1}{m-1} \ln \frac{1}{d} \\
& +\ln \left[(t-1) \exp \left(a \sum_{k=1}^{m-2} v_{k}\right)+\exp \left(-a v_{m-1}\right)\right]+O\left(d^{\sigma}\right) .
\end{aligned}
$$

И наконец, при $t_{0}+1 \leqslant t \leqslant T_{0}$ получаем асимптотические представления

$$
\begin{aligned}
y_{j}(t, v, d)= & \frac{1}{a(m-1)} \ln \frac{1}{d}+v_{j}+d^{\sigma}\left(t-t_{0}-1-\frac{1}{a}\right) \\
& \quad \times\left(\exp v_{j+1}-\exp v_{j}\right)+o\left(d^{\sigma}\right), \quad j=1, \ldots, m-2 ; \\
y_{m-1}(t, v, d)= & \frac{1}{a(m-1)} \ln \frac{1}{d} \\
& +\ln \left[t_{0} \exp \left(a \sum_{k=1}^{m-2} v_{k}\right)+\exp \left(-a v_{m-1}\right)\right] \\
& -\left(1+\frac{1}{a}\right) \ln \left[\left(t_{0}-1\right) \exp \left(a \sum_{k=1}^{m-2} v_{k}\right)+\exp \left(-a v_{m-1}\right)\right]+O\left(d^{\sigma}\right) .
\end{aligned}
$$

Добавим еще, что во всех формулах (3.89)-(3.94) остатки имеют указанный порядок малости равномерно по $t, v$ и сохраняют его при дифференцировании по $v$. 
Из приведенного асимптотического анализа следует, что в случае (3.88) отображение (3.27) записывается в виде

$$
\begin{aligned}
v_{j} \rightarrow v_{j}+d^{\sigma}\left(a-\frac{1}{a}\right)\left(\exp v_{j+1}-\exp v_{j}\right)+o\left(d^{\sigma}\right), \quad j=1, \ldots, m-2 \\
v_{m-1} \rightarrow \ln \left[t_{0} \exp \left(a \sum_{k=1}^{m-2} v_{k}\right)+\exp \left(-a v_{m-1}\right)\right] \\
-\left(1+\frac{1}{a}\right) \ln \left[\left(t_{0}-1\right) \exp \left(a \sum_{k=1}^{m-2} v_{k}\right)+\exp \left(-a v_{m-1}\right)\right]+O\left(d^{\sigma}\right) .
\end{aligned}
$$

Заметим, далее, что при $d=0$ в силу уже известных свойств вспомогательного отображения (3.54) (см. лемму 3.2) отображение (3.95), (3.96) допускает экспоненциально устойчивое $(m-2)$-мерное инвариантное многообразие, состоящее из неподвижных точек. Упомянутое многообразие задается равенством

$$
\begin{gathered}
\left\{\left(v_{1}, \ldots, v_{m-1}\right): v_{m-1}=v_{*}-v_{1}-v_{2}-\cdots-v_{m-2},\right. \\
\left.v_{j} \in \mathbb{R}, j=1, \ldots, m-2\right\}
\end{gathered}
$$

где $v=v_{*}$ - единственный корень уравнения

$$
v=\ln \left[t_{0}+\exp (-a v)\right]-\left(1+\frac{1}{a}\right) \ln \left[t_{0}-1+\exp (-a v)\right] .
$$

При малых $d>0$ аналог инвариантного многообразия (3.97) сохраняется, а сужение на него отображения (3.95), (3.96) представляет собой оператор, асимптотически близкий к тождественному. В связи с этим указанный оператор можно аппроксимировать соответствующей системой обыкновенных дифференциальных уравнений. В нашем случае эта система имеет вид

$$
\begin{aligned}
\frac{d v_{j}}{d \tau} & =\exp v_{j+1}-\exp v_{j}, \quad j=1, \ldots, m-3, \\
\frac{d v_{m-2}}{d \tau} & =\exp \left(v_{*}-v_{1}-v_{2}-\cdots-v_{m-2}\right)-\exp v_{m-2} .
\end{aligned}
$$

Ясно также, что любому ее экспоненциально устойчивому состоянию равновесия $\mathscr{O}$ в исходном отображении (3.95), (3.96) при всех достаточно малых $d>0$ отвечает устойчивая неподвижная точка $\mathscr{O}(d)$, асимптотически близкая к $\mathscr{O}$.

Анализ системы (3.98) не вызывает затруднений. Действительно, заменами $v_{j}=w_{j}+v_{*} /(m-1), j=1, \ldots, m-2, \tau \exp \left(v_{*} /(m-1)\right) \rightarrow \tau$ она приводится к виду

$$
\begin{gathered}
\dot{w}_{j}=\exp w_{j+1}-\exp w_{j}, \quad j=1, \ldots, m-2, \\
w_{m-1}=-w_{1}-w_{2}-\cdots-w_{m-2} .
\end{gathered}
$$

А отсюда и из отмеченных выше свойств аналогичной системы (3.78) вытекает, что нулевое состояние равновесия системы (3.99) глобально экспоненциально устойчиво. 
Возвращаясь к отображению (3.27), приходим к выводу, что при условии (2.61) и при всех $d \ll 1$ оно допускает экспоненциально устойчивую неподвижную точку

$$
\begin{gathered}
z_{* *}=\left(z_{1}^{* *}, \ldots, z_{m-1}^{* *}\right), \quad z_{j}^{* *}=\frac{1}{a(m-1)} \ln \frac{1}{d}+\frac{v_{*}}{m-1}+o(1), \\
j=1, \ldots, m-1,
\end{gathered}
$$

а также серию устойчивых неподвижных точек

$$
z_{* *}^{(k)}=\mathscr{A}^{k} z_{* *}, \quad k=1, \ldots, m-1 .
$$

Как и в случае (3.85), (3.87), в исходной системе (3.73) неподвижным точкам (3.100), (3.101) соответствуют устойчивые релаксационные периодические движения, переходящие друг в друга при циклических перестановках компонент $u_{j}, j=1, \ldots, m$. Добавим еще, что при условии (3.79) найденные нами две группы устойчивых периодических решений сосуществуют.

3.4. Случай полносвязной нейронной сети. В настоящем пункте разработанные нами методы исследования периодических решений распространяются на полносвязную нейронную сеть. А именно, рассматривается аналогичная (1.38) система

$$
\dot{u}_{j}=d \sum_{i=1}^{m}\left(u_{i}-u_{j}\right)+\lambda f\left(u_{j}(t-1)\right) u_{j}, \quad j=1, \ldots, m,
$$

с симметричными нейронными связями. Здесь $\lambda \gg 1, d=$ const $>0$, a функция $f(u)$ удовлетворяет условиям (1.49), (1.50), (2.61).

Не претендуя на полноту анализа, ввиду сложности системы (3.102) ограничимся изучением специальных ее периодических решений - так называемых режимов двухкластерной синхронизации.

Для описания упомянутых режимов предположим, что совокупность индексов $1 \leqslant j \leqslant m$ разбита на два непересекающихся множества $\Sigma_{1}, \Sigma_{2}$, т. е.

$$
\{1,2, \ldots, m\}=\Sigma_{1} \cup \Sigma_{2} .
$$

Далее, обозначим через $k$ и $m-k$ количества элементов в множествах $\Sigma_{1}$ и $\Sigma_{2}$ соответственно. Тогда, очевидно, система (3.102) допускает решения с компонентами

$$
u_{j}=w_{1}(t) \quad \text { при } j \in \Sigma_{1}, \quad u_{j}=w_{2}(t) \quad \text { при } j \in \Sigma_{2}, \quad w_{1}(t) \not \equiv w_{2}(t),
$$

где переменные $w_{1}, w_{2}$ удовлетворяют системе

$$
\begin{aligned}
& \dot{w}_{1}=d\left(k w_{1}+(m-k) w_{2}-m w_{1}\right)+\lambda f\left(w_{1}(t-1)\right) w_{1}, \\
& \dot{w}_{2}=d\left(k w_{1}+(m-k) w_{2}-m w_{2}\right)+\lambda f\left(w_{2}(t-1)\right) w_{2} .
\end{aligned}
$$

Если же, в свою очередь, система (3.105) имеет периодическое решение, то отвечающее ему решение (3.104) исходной системы (3.102) назовем периодическим режимом двухкластерной синхронизации. 
Все допустимые типы периодических решений двухкластерной синхронизации, отвечающие произвольным разбиениям (3.103), будем кодировать с помощью $m$-мерных символьных векторов вида

$$
\left(\vartheta_{1}, \vartheta_{2}, \ldots, \vartheta_{m}\right): \quad \vartheta_{j}=A \text { или } B, \quad j=1, \ldots, m .
$$

Точнее говоря, предполагаем, что $j$-я координата вектора (3.106) равна $A$ в случае $j \in \Sigma_{1}$ и $B$ в случае $j \in \Sigma_{2}$. При таком способе кодирования однородному циклу (3.2) будут соответствовать сразу два вектора $(A, A, \ldots, A)$ и $(B, B, \ldots, B)$. В связи с этим данные кодировки считаем совпадающими.

Обращаем внимание, что всем векторам (3.106) с наперед заданными количествами элементов $A$ и $B$, равными $k$ и $m-k$, отвечает одна и та же система (3.105). Как будет показано ниже, при достаточно малых фиксированных значениях $d>0$ и при всех $\lambda \gg 1$ эта система допускает два неоднородных цикла

$$
\begin{array}{ll}
C_{1}: & \left(w_{1}, w_{2}\right)=\left(w_{1}^{(k, m-k)}(t), w_{2}^{(k, m-k)}(t)\right), \\
C_{2}: & \left(w_{1}, w_{2}\right)=\left(w_{2}^{(m-k, k)}(t), w_{1}^{(m-k, k)}(t)\right),
\end{array}
$$

переходящих друг в друга при заменах $k \rightarrow m-k, w_{1} \rightarrow w_{2}, w_{2} \rightarrow w_{1}$. А так как порядок следования переменных $w_{1}, w_{2}$ для нас несущественен, то для определенности всегда будем считать, что функции $w_{1}(t), w_{2}(t)$ из (3.104) заданы, к примеру, равенствами

$$
w_{1}(t)=w_{1}^{(k, m-k)}(t), \quad w_{2}(t)=w_{2}^{(k, m-k)}(t) .
$$

Отметим, что при описанных выше способах кодирования и отыскания компонент $w_{1}(t), w_{2}(t)$ имеет место взаимно однозначное соответствие между множеством всех периодических режимов двухкластерной синхронизации и множеством символьных векторов (3.106). Таким образом, с учетом однородного цикла (3.2) существует ровно $2^{m}-1$ двухкластерных режимов. Как выяснится в последующем, при достаточно малых значениях параметра $d$ все они экспоненциально орбитально устойчивы.

Сделаем еще одно полезное наблюдение. Несложная проверка показывает, что система (3.102) инвариантна относительно замен

$$
\left(u_{1}, u_{2}, \ldots, u_{m}\right) \rightarrow\left(u_{j_{1}}, u_{j_{2}}, \ldots, u_{j_{m}}\right)
$$

где $\left(j_{1}, j_{2}, \ldots, j_{m}\right)$ - произвольная перестановка набора индексов $\{1,2, \ldots, m\}$. А это значит, что если мы найдем циклы (3.104), (3.108), соответствующие символьным векторам

$$
(\underbrace{A, \ldots, A}_{k}, \underbrace{B, \ldots, B}_{m-k})
$$

при каждом $k, 1 \leqslant k \leqslant m-1$, и применим к ним преобразования (3.109), то в итоге будут исчерпаны все периодические режимы двухкластерной синхронизации, отличные от однородного цикла.

Как и в случае системы (3.73), для полносвязной сети (3.102) остаются в силе теоремы 3.1, 3.2. Точнее говоря, после замен (3.3) здесь получается некоторая система для $x, y_{1}, \ldots, y_{m-1}$, аналогичная системам (3.4), (3.5) 
и $(3.74),(3.75)$. Явный вид этой системы опустим. Отметим только, что для оператора последования $\Pi_{\varepsilon}(\varphi)$ (см. (3.8)-(3.10)) по ее траекториям сохраняются равенства (3.21). А отсюда, в свою очередь, следует, что за существование и устойчивость ее периодических решений отвечает в конечном итоге аналогичное $(3.27)(m-1)$-мерное отображение, где теперь $\left(y_{1}^{0}(t, z), \ldots, y_{m-1}^{0}(t, z)\right)-$ решение задачи Коши

$$
\begin{gathered}
\dot{y}_{j}=d\left[\sum_{r=1}^{j} \exp \left(-\sum_{s=r}^{j} y_{s}\right)+\sum_{r=j+2}^{m} \exp \left(\sum_{s=j+1}^{r-1} y_{s}\right)\right. \\
\left.-\sum_{r=1}^{j-1} \exp \left(-\sum_{s=r}^{j-1} y_{s}\right)-\sum_{r=j+1}^{m} \exp \left(\sum_{s=j}^{r-1} y_{s}\right)\right], \\
y_{j}(1+0)=y_{j}(1-0)-(1+a) y_{j}(0), \quad j=1, \ldots, m-1, \\
y_{j}\left(t_{0}+1+0\right)=y_{j}\left(t_{0}+1-0\right)-\left(1+\frac{1}{a}\right) y_{j}\left(t_{0}\right), \quad z=\left(z_{1}, \ldots, z_{m-1}\right) .
\end{gathered}
$$

Добавим еще, что все суммы в (3.111), у которых нижний индекс больше верхнего, считаются равными нулю.

Проблема корректности отображения (3.27) в данном случае решается так же, как и в двух предыдущих. А именно, это отображение заведомо определено во всем пространстве $\mathbb{R}^{m-1}$, поскольку все решения вспомогательной системы (3.111) существуют на полуоси $t \geqslant 0$ и стремятся к нулю при $t \rightarrow+\infty$. Действительно, любое ее решение допускает представление вида (3.15), где $\left(\xi_{1}, \ldots, \xi_{m}\right)$ - решение системы

$$
\dot{\xi}_{j}=d\left(\sum_{i=1}^{m} \xi_{i}-m \xi_{j}\right), \quad j=1, \ldots, m
$$

лежащее в инвариантном конусе $K$ векторов с положительными координатами. А так как для упомянутых решений системы (3.114) сохраняются соотношения (3.17), то отсюда вытекают требуемые факты.

Обратимся теперь к интересующему нас вопросу о существовании у системы (3.102) циклов двухкластерной синхронизации и их устойчивости. Как уже было сказано выше, данная проблема сводится к исследованию лишь специальных циклов этого семейства, закодированных векторами (3.110). Для таких циклов очевидным образом имеем

$$
y_{j}=\ln \frac{u_{j+1}}{u_{j}} \equiv 0 \quad \text { при } j=1, \ldots, m-1, j \neq k ; \quad y_{k} \not \equiv 0 .
$$

Подставляя, далее, соотношения (3.115) в (3.111)-(3.113), приходим к выводу, что от исходного отображения (3.27) "отщепляется" одномерное отображение вида

$$
z_{k} \rightarrow \Phi_{0, k}\left(z_{k}\right)=\left.y_{k}^{0}\left(t, z_{k}\right)\right|_{t=T_{0}}
$$


где $y_{k}^{0}\left(t, z_{k}\right)$ - решение импульсной задачи Коши

$$
\begin{gathered}
\dot{y}=d(k \exp (-y)+m-2 k-(m-k) \exp y), \\
y(1+0)=y(1-0)-(1+a) y(0), \\
y\left(t_{0}+1+0\right)=y\left(t_{0}+1-0\right)-\left(1+\frac{1}{a}\right) y\left(t_{0}\right), \\
\left.y\right|_{t=0}=z_{k} .
\end{gathered}
$$

Добавим еще, что получившееся одномерное отображение в силу своего происхождения отвечает за существование и устойчивость циклов вспомогательной системы (3.105).

Как и во всех предыдущих случаях, отыскание неподвижных точек отображения (3.116) проведем в предположении о малости $d$. Следуя изложенной в п. 3.2 методике, подставим в (3.118) соотношение

$$
z_{k}=\frac{1}{a} \ln \frac{1}{d}+v, \quad v=\text { const } \in \mathbb{R},
$$

и обозначим через $y_{k}(t, v, d)$ решение получившейся задачи Коши (3.117)-(3.119). Проводя соответствующие подсчеты, убеждаемся, что при $d \rightarrow 0$ справедливы следующие асимптотические представления:

$$
\begin{array}{ll}
y_{k}=\frac{1}{a} \ln \frac{1}{d}+v+O\left(d^{1-1 / a}\right) & \text { при } 0 \leqslant t<1 ; \\
y_{k}=-\ln \frac{1}{d}+\ln [k(t-1)+\exp (-a v)]+O\left(d^{1-1 / a}\right) & \text { при } 1 \leqslant t<t_{0}+1 ; \\
y_{k}=\frac{1}{a} \ln \frac{1}{d}+\psi_{k}(v)+O\left(d^{1-1 / a}\right) & \text { при } t_{0}+1 \leqslant t \leqslant T_{0},
\end{array}
$$

где

$$
\psi_{k}(v)=\ln \left[k t_{0}+\exp (-a v)\right]-\left(1+\frac{1}{a}\right) \ln \left[k\left(t_{0}-1\right)+\exp (-a v)\right] .
$$

Соотношения (3.120)-(3.122) свидетельствуют о том, что после выполнения в отображении (3.116) замены (3.119) оно допускает при $d \rightarrow 0$ (в $C^{1}$-метрике на любом конечном отрезке изменения $v$ ) предел

$$
v \rightarrow \psi_{k}(v)
$$

Далее, из (3.123) заключаем, что отображение (3.124) - частный случай уже изученного ранее отображения (3.54) (при $r_{1}=k t_{0}, r_{2}=k\left(t_{0}-1\right)$ ). А так как требование (3.55) здесь выполняется (оно эквивалентно неравенству (2.61)), то отображение (3.124) имеет единственную экспоненциально устойчивую неподвижную точку $v=v_{*, k}$. В исходном отображении (3.116) этой неподвижной точке отвечает устойчивая неподвижная точка

$$
z_{k}=z_{k}^{*}, \quad z_{k}^{*}=\frac{1}{a} \ln \frac{1}{d}+v_{*, k}+O\left(d^{1-1 / a}\right), \quad d \rightarrow 0,
$$

а в системе $(3.105)$ - устойчивый релаксационный цикл $C_{1}$ (см. (3.107)) 
Следует также заметить, что в силу инвариантности системы (3.117) по отношению к преобразованиям $k \rightarrow m-k, y \rightarrow-y$ наряду с точкой $(3.125)$ отображение (3.116) допускает также устойчивую неподвижную точку $z_{k}=z_{k}^{* *}$ c асимптотикой

$$
z_{k}^{* *}=-\frac{1}{a} \ln \frac{1}{d}-v_{*, m-k}+O\left(d^{1-1 / a}\right), \quad d \rightarrow 0 .
$$

Этой неподвижной точке в системе (3.105) соответствует цикл $C_{2}$ (см. (3.107)), не представляющий для нас интереса, поскольку выше мы договорились определять компоненты $w_{1}(t), w_{2}(t)$ из (3.104) по правилам (3.108).

Итак, для каждого натурального $k, 1 \leqslant k \leqslant m-1$, удалось найти цикл типа двухкластерной синхронизации, ассоциированный с символьным вектором (3.110). Согласно развитой нами теории, за устойчивость этого цикла отвечает расположение спектра матрицы Якоби $\Phi_{0}^{\prime}\left(\widetilde{z}_{(k)}\right)$ отображения (3.27) в неподвижной точке $\widetilde{z}_{(k)}=\left(z_{1}, \ldots, z_{m-1}\right): z_{j}=0$ при $j \neq k, z_{k}=z_{k}^{*}$, где $z_{k}^{*}-$ неподвижная точка (3.125) отображения (3.116).

Одно собственное значение матрицы $\Phi_{0}^{\prime}\left(\widetilde{z}_{(k)}\right)$ заранее известно - это мультипликатор неподвижной точки (3.125), задаваемый равенством

$$
\mu=\left.\frac{d \Phi_{0, k}}{d z_{k}}\right|_{z_{k}=z_{k}^{*}}=\left.\frac{d \psi_{k}}{d v}\right|_{v=v_{*, k}}+O\left(d^{1-1 / a}\right), \quad d \rightarrow 0,
$$

и в силу отмеченных выше свойств отображения (3.124) принадлежащий интервалу $(0,1)$. Другие же собственные значения данной матрицы совпадают со спектром оператора сдвига $Г$ за время от $t=0$ до $t=T_{0}$ по решениям импульсной системы:

$$
\begin{aligned}
& \dot{\mathrm{g}}_{j}=d\left[-\sum_{r=1}^{j} \sum_{s=r}^{j} \mathrm{~g}_{s}+\sum_{r=j+2}^{m} \sum_{s=j+1}^{r-1} \mathrm{~g}_{s}+\exp \left(y_{k}^{*}\right) \sum_{r=k+1}^{m} \sum_{\substack{s=j+1 \\
s \neq k}}^{r-1} \mathrm{~g}_{s}\right. \\
& \left.+\sum_{r=1}^{j-1} \sum_{s=r}^{j-1} \mathrm{~g}_{s}-\sum_{r=j+1}^{k} \sum_{s=j}^{r-1} \mathrm{~g}_{s}-\exp \left(y_{k}^{*}\right) \sum_{r=k+1}^{m} \sum_{\substack{s=j \\
s \neq k}}^{r-1} \mathrm{~g}_{s}\right], \quad 1 \leqslant j<k \\
& \dot{\mathrm{g}}_{j}=d\left[-\exp \left(-y_{k}^{*}\right) \sum_{r=1}^{k} \sum_{\substack{s=r \\
s \neq k}}^{j} \mathrm{~g}_{s}-\sum_{r=k+1}^{j} \sum_{s=r}^{j} \mathrm{~g}_{s}+\sum_{r=j+2}^{m} \sum_{s=j+1}^{r-1} \mathrm{~g}_{s}\right. \\
& \left.+\exp \left(-y_{k}^{*}\right) \sum_{r=1}^{k} \sum_{\substack{s=r \\
s \neq k}}^{j-1} \mathrm{~g}_{s}+\sum_{r=k+1}^{j-1} \sum_{s=r}^{j-1} \mathrm{~g}_{s}-\sum_{r=j+1}^{m} \sum_{s=j}^{r-1} \mathrm{~g}_{s}\right], \quad k<j \leqslant m-1, \\
& \mathrm{~g}_{j}(1+0)=\mathrm{g}_{j}(1-0)-(1+a) \mathrm{g}_{j}(0), \\
& \mathrm{g}_{j}\left(t_{0}+1+0\right)=\mathrm{g}_{j}\left(t_{0}+1-0\right)-\left(1+\frac{1}{a}\right) \mathrm{g}_{j}\left(t_{0}\right) \text {, } \\
& j=1, \ldots, m-1, \quad j \neq k \text {. }
\end{aligned}
$$


Здесь функция $y_{k}^{*}$ является решением задачи Коши (3.117), (3.118) при $z_{k}=z_{k}^{*}$ и вследствие этого допускает представления вида (3.120)-(3.122) (в которых $v$ и $\psi_{k}(v)$ необходимо заменить на неподвижную точку $v_{*, k}$ отображения (3.124)).

Для вычисления спектра введенного выше оператора $Г$ дополним систему (3.126)-(3.128) произвольными не зависящими от $d$ начальными условиями $\left.\mathrm{g}_{j}\right|_{t=0}=\mathrm{g}_{j}^{0}, j=1, \ldots, m-1, j \neq k$, и предпримем асимптотическое интегрирование получившейся задачи Коши.

Как обычно, начнем с промежутка $0 \leqslant t<1$. Опираясь на формулу (3.120), приходим к выводу, что в данном случае система (3.126), (3.127) записывается в виде

$$
\begin{gathered}
\dot{\mathrm{g}}_{j}=d^{1-1 / a} \exp \left(v_{*, k}\right)\left(\sum_{r=k+1}^{m} \sum_{\substack{s=j+1 \\
s \neq k}}^{r-1} \mathrm{~g}_{s}-\sum_{r=k+1}^{m} \sum_{\substack{s=j \\
s \neq k}}^{r-1} \mathrm{~g}_{s}\right)+o\left(d^{1-1 / a}\right) \\
=-d^{1-1 / a}(m-k) \mathrm{g}_{j} \exp \left(v_{*, k}\right)+o\left(d^{1-1 / a}\right), \quad j<k ; \\
\dot{\mathrm{g}}_{j}=O(d), \quad j>k, \quad d \rightarrow 0 .
\end{gathered}
$$

А отсюда, в свою очередь, выводим:

$$
\begin{gathered}
\mathrm{g}_{j}=\mathrm{g}_{j}^{0}\left(1-d^{1-1 / a} t(m-k) \exp \left(v_{*, k}\right)\right)+o\left(d^{1-1 / a}\right), \quad j<k ; \\
\mathrm{g}_{j}=\mathrm{g}_{j}^{0}+O(d), \quad j>k, \quad d \rightarrow 0 .
\end{gathered}
$$

Следует особо подчеркнуть, что для краткости символами $o\left(d^{1-1 / a}\right), O(d)$ в формулах (3.129)-(3.131) и в последующих формулах для $\dot{\mathrm{g}}_{j}, \mathrm{~g}_{j}$ обозначаются некоторые линейные формы переменных $\mathrm{g}_{s}, s=1, \ldots, m-1, s \neq k$ (в случае $\dot{\mathrm{g}}_{j}$ ) или $\mathrm{g}_{s}^{0}, s=1, \ldots, m-1, s \neq k$ (в случае $\mathrm{g}_{j}$ ) с коэффициентами, зависящими от $t$ и $d$. Что же касается упомянутых коэффициентов, то именно они равномерно по $t$ имеют указанные порядки малости при $d \rightarrow 0$.

При $1 \leqslant t<t_{0}+1$, учитывая равенство (3.121) и уже установленные формулы (3.131), получаем задачу Коши

$$
\begin{aligned}
& \dot{\mathrm{g}}_{j}=O(d), \quad j<k \text {; }
\end{aligned}
$$

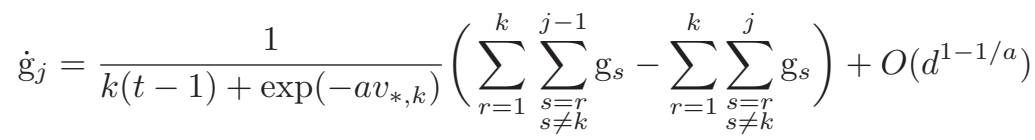

$$
\begin{aligned}
& =-\frac{k \mathrm{~g}_{j}}{k(t-1)+\exp \left(-a v_{*, k}\right)}+O\left(d^{1-1 / a}\right), \quad j>k \text {; } \\
& \left.\mathrm{g}_{j}\right|_{t=1}=-\mathrm{g}_{j}^{0}\left(a+d^{1-1 / a}(m-k) \exp \left(v_{*, k}\right)\right)+o\left(d^{1-1 / a}\right), \quad j<k ; \\
& \left.\mathrm{g}_{j}\right|_{t=1}=-a \mathrm{~g}_{j}^{0}+O(d), \quad j>k, \quad d \rightarrow 0 \text {. }
\end{aligned}
$$

Несложный ее анализ приводит к асимптотическим представлениям

$$
\begin{array}{ll}
\mathrm{g}_{j}=-\mathrm{g}_{j}^{0}\left(a+d^{1-1 / a}(m-k) \exp \left(v_{*, k}\right)\right)+o\left(d^{1-1 / a}\right), & j<k ; \\
\mathrm{g}_{j}=-\frac{a \exp \left(-a v_{*, k}\right)}{k(t-1)+\exp \left(-a v_{*, k}\right)} \mathrm{g}_{j}^{0}+O\left(d^{1-1 / a}\right), & j>k, \quad d \rightarrow 0 .
\end{array}
$$


На последнем участке $t_{0}+1 \leqslant t \leqslant T_{0}$, объединяя формулы $(3.122)$ и (3.132), снова приходим к системе вида (3.129), (3.130), но уже с начальными условиями

$$
\begin{array}{ll}
\left.\mathrm{g}_{j}\right|_{t=t_{0}+1}=\mathrm{g}_{j}^{0}\left(1+\frac{d^{1-1 / a}}{a}(m-k) \exp \left(v_{*, k}\right)\right)+o\left(d^{1-1 / a}\right), & j<k ; \\
\left.\mathrm{g}_{j}\right|_{t=t_{0}+1}=\left.\mathrm{g}_{j}^{0} \frac{d \psi_{k}}{d v}\right|_{v=v_{*, k}}+O\left(d^{1-1 / a}\right), & j>k, \quad d \rightarrow 0,
\end{array}
$$

где, напомним, $\psi_{k}(v)$ - функция (3.123). Проводя асимптотическое интегрирование задачи Коши (3.129), (3.130), (3.133), убеждаемся, что

$$
\begin{array}{rlrl}
\mathrm{g}_{j}=\mathrm{g}_{j}^{0}\left[1-d^{1-1 / a}(m-k)\left(t-t_{0}-1-\frac{1}{a}\right) \exp \left(v_{*, k}\right)\right] & \\
& +o\left(d^{1-1 / a}\right), & j<k ; \\
\mathrm{g}_{j}=\left.\mathrm{g}_{j}^{0} \frac{d \psi_{k}}{d v}\right|_{v=v_{*, k}}+O\left(d^{1-1 / a}\right), & j>k, \quad d \rightarrow 0 .
\end{array}
$$

Подводя итог, заметим, что интересующий нас оператор Г задается соотношением

$$
\left.\left(\mathrm{g}_{1}^{0}, \ldots, \mathrm{g}_{k-1}^{0}, \mathrm{~g}_{k+1}^{0}, \ldots, \mathrm{g}_{m-1}^{0}\right) \rightarrow\left(\mathrm{g}_{1}, \ldots, \mathrm{g}_{k-1}, \mathrm{~g}_{k+1}, \ldots, \mathrm{g}_{m-1}\right)\right|_{t=T_{0}} .
$$

Подставляя, далее, в (3.135) формулы (3.134), приходим к выводу, что собственные значения оператора $Г$ допускают при $d \rightarrow 0$ асимптотику

$$
\begin{array}{ll}
\mu_{j}=1-\left(a-\frac{1}{a}\right) d^{1-1 / a}(m-k) \exp \left(v_{*, k}\right)+o\left(d^{1-1 / a}\right), & j=1, \ldots, k-1 \\
\mu_{j}=\left.\frac{d \psi_{k}}{d v}\right|_{v=v_{*, k}}+O\left(d^{1-1 / a}\right), & j=k, \ldots, m-2,
\end{array}
$$

и вследствие этого удовлетворяют неравенствам $\left|\mu_{j}\right|<1, j=1, \ldots, m-2$.

Итак, все найденные нами периодические режимы системы (3.102) типа двухкластерной синхронизации, отличные от однородного цикла (3.2), при малых фиксированных $d$ и при $\lambda \gg 1$ экспоненциально орбитально устойчивы. Что же касается однородного цикла, то он не является исключением, поскольку отвечающая ему неподвижная точка $z=0$ отображения (3.27) по-прежнему экспоненциально устойчива при всех $d>0$.

Действительно, рассуждая, как при доказательстве леммы 3.2, приходим к выводу, что собственные значения матрицы Якоби $\Phi_{0}^{\prime}(0)$ задаются равенствами $\mu_{k}=\left.\mu(s)\right|_{s=s_{k}}, k=1, \ldots, m-1$, где $\mu(s)-$ функция $(3.33), s=s_{k}-$ ненулевые собственные значения матрицы системы (3.114). А так как $s_{k}=-m$, $k=1, \ldots, m-1$, то в силу свойств $(3.34)$ имеем $\mu_{k} \in(0,1), k=1, \ldots, m-1$.

3.5. Об одном способе математического моделирования химических синапсов. Предположим, что имеет место однонаправленное синаптическое взаимодействие в сети из $m, m \geqslant 2$, нейронов, объединенных в кольцо, причем каждый отдельно взятый нейрон моделируется уравнением (1.51) 
при условиях $(1.49),(1.50)$ на функцию $f(u)$. Тогда, руководствуясь описанной в п. 1.5 методикой, мы можем перейти от уравнения (1.51) к аналогичной (1.39) системе

$$
\dot{u}_{j}=\lambda f\left(u_{j}(t-1)\right) u_{j}+b s_{j-1}\left(u_{j-1}\right)\left(u_{*}-u_{j}\right), \quad j=1, \ldots, m,
$$

где $u_{0}=u_{m}, s_{0}=s_{m}$, а функции $s_{j}$ заданы равенствами (1.40). Однако, на наш взгляд, в данной ситуации следует отказаться от общепринятых представлений и в качестве математической модели рассматриваемой нейронной сети взять несколько иную систему

$$
\dot{u}_{j}=\left[\lambda f\left(u_{j}(t-1)\right)+b g\left(u_{j-1}\right) \ln \frac{u_{*}}{u_{j}}\right] u_{j}, \quad j=1, \ldots, m, \quad u_{0}=u_{m},
$$

в которой $b=$ const $>0, u_{*}=\exp (c \lambda), c=$ const $\in \mathbb{R}$, а функция $g(u) \in C^{2}\left(\mathbb{R}_{+}\right)$ такова, что

$$
\begin{gathered}
g(u)>0 \quad \forall u>0, \quad g(0)=0 ; \\
g(u)-1, u g^{\prime}(u), u^{2} g^{\prime \prime}(u)=O\left(\frac{1}{u}\right) \quad \text { при } u \rightarrow+\infty .
\end{gathered}
$$

Мотивы, по которым мы остановили свой выбор на системе (3.137), состоят в следующем. Во-первых, при переходе от (3.136) к (3.137) общий качественный характер синаптической связи сохраняется, поскольку в обоих случаях соответствующие связующие слагаемые $b s_{j-1}\left(u_{j-1}\right)\left(u_{*}-u_{j}\right)$ и $b g\left(u_{j-1}\right) u_{j} \ln \left(u_{*} / u_{j}\right)$ меняют знак с "+" на “-" при увеличении потенциалов $u_{j}$ и при прохождении их через критическое значение $u_{*}$. Во-вторых, и это самое главное, для системы (3.137) удается корректно определить предельный объект при $\lambda \rightarrow+\infty$, которым оказывается некоторая релейная система с запаздыванием.

Действительно, после перехода к переменным $x_{j}=(1 / \lambda) \ln u_{j}, j=1, \ldots, m$, система (3.137) записывается в виде

$$
\dot{x}_{j}=F\left(x_{j}(t-1), \varepsilon\right)+b\left(c-x_{j}\right) G\left(x_{j-1}, \varepsilon\right), \quad j=1, \ldots, m,
$$

где $\varepsilon=1 / \lambda \ll 1, x_{0}=x_{m}, F(x, \varepsilon)=f(\exp (x / \varepsilon)), G(x, \varepsilon)=g(\exp (x / \varepsilon))$. Далее, обратим внимание, что в силу свойств (1.49), (3.138) справедливы предельные равенства

$$
\lim _{\varepsilon \rightarrow 0} F(x, \varepsilon)=R(x), \quad \lim _{\varepsilon \rightarrow 0} G(x, \varepsilon)=H(x),
$$

где $R(x), H(x)$ - функции из (2.4) и (1.40) соответственно. А отсюда, в свою очередь, следует, что при $\varepsilon \rightarrow 0$ система (3.139) переходит в релейную систему

$$
\dot{x}_{j}=R\left(x_{j}(t-1)\right)+b\left(c-x_{j}\right) H\left(x_{j-1}\right), \quad j=1, \ldots, m, \quad x_{0}=x_{m} .
$$

Интересно также отметить, что в предельной системе (3.140) связующие слагаемые $b\left(c-x_{j}\right) H\left(x_{j-1}\right)$ уже в точности совпадают с соответствующими слагаемыми из (3.136). Тем самым, наблюдается некая преемственность по отношению к общепринятому способу моделирования химических синапсов. 
Наличие предельного объекта (3.140) существенно облегчает проблему отыскания аттракторов системы (3.139) и позволяет, в частности, применить к ней общие результаты из [48] о соответствии между устойчивыми циклами релейной и релаксационной систем. Опираясь на эти результаты, в работе [50] было показано, что при увеличении $m$ и при подходящем выборе остальных параметров в системе (3.139) может сосуществовать любое конечное число устойчивых периодических движений типа бегущих волн. Точнее говоря, в [50] речь шла о сосуществовании устойчивых периодических решений, допускающих представление

$$
x_{j}=x(t+(j-1) \Delta, \varepsilon), \quad j=1, \ldots, m,
$$

где $\Delta>0$, а функция $x(t, \varepsilon)$ - периодическое решение вспомогательного уравнения

$$
\dot{x}=F(x(t-1), \varepsilon)+b(c-x) G(x(t-\Delta), \varepsilon)
$$

периода $T=m \Delta / k, k \in \mathbb{N}$.

\section{4. Модель импульсного нейрона с двумя запаздываниями}

4.1. Математическое описание bursting-эффекта. В настоящем разделе в качестве математической модели отдельного нейрона рассматривается уравнение (1.48) с двумя запаздываниями при условиях (1.49), (1.50) на функции $f(u), g(u)$. Как оказывается, наличие второго запаздывания существенно меняет свойства модели и позволяет, в частности, охватить такие явления, как bursting-эффект и буферность.

Обратимся сначала к bursting-эффекту. Как уже отмечалось в п. 1.3, для моделирования этого феномена обычно привлекают трехмерные релаксационные системы вида (1.13). Мы же установим, что bursting-эффект наблюдается в рамках уравнения (1.48). Точнее говоря, ниже показывается следующее: по любому фиксированному натуральному $n$ можно так подобрать фигурирующие в (1.48)-(1.50) параметры $h, a, b$, что при всех достаточно больших $\lambda$ уравнение (1.48) будет иметь экспоненциально орбитально устойчивый цикл $u=u_{*}(t, \lambda)$ периода $T_{*}(\lambda)$, где $T_{*}(\lambda)$ при $\lambda \rightarrow \infty$ стремится к некоторому конечному пределу $T_{*}>0$. При этом сама функция $u_{*}(t, \lambda)$ на отрезке времени длины периода допускает ровно $n$ подряд идущих асимптотически высоких (порядка $\exp (\lambda h))$ всплесков продолжительности $\Delta t=(1+1 / a) h$, а все остальное время она асимптотически мала. Иными словами, при указанном выборе параметров $h, a, b$ реализуется bursting-эффект.

Наглядное представление о релаксационных свойствах цикла $u_{*}(t, \lambda)$ дает его график на плоскости $(t, u)$ (см. рис. 8), построенный в масштабе 25:1 для значений параметров $h=1 / 26, \lambda=130$ и для функций $f(u)=(1-u) /(1+0.5 u)$, $g(u)=4 u /(1+u)$.

Как и в п. 2.1, при исследовании вопроса о существовании и устойчивости у уравнения (1.48) релаксационного bursting-цикла целесообразно перейти к новой переменной $x$ по формуле $u=\exp (\lambda x)$. Указанная замена преобразует интересующее нас уравнение к аналогичному (2.3) виду

$$
\dot{x}=F(x(t-h), \varepsilon)-G(x(t-1), \varepsilon),
$$




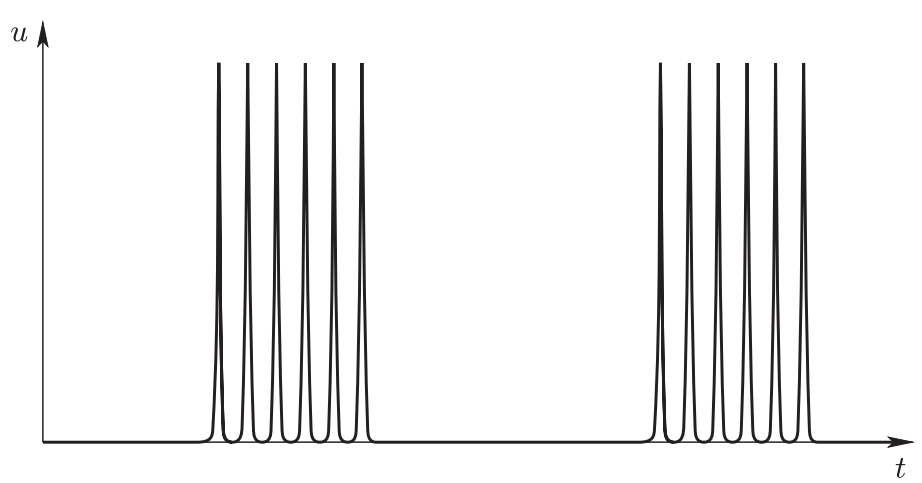

Рис. 8

где $F(x, \varepsilon)=f(\exp (x / \varepsilon)), G(x, \varepsilon)=g(\exp (x / \varepsilon)), \varepsilon=1 / \lambda \ll 1$. Далее, из свойств (1.49), (1.50) вытекает, что

$$
\lim _{\varepsilon \rightarrow 0} F(x, \varepsilon)=R(x), \quad \lim _{\varepsilon \rightarrow 0} G(x, \varepsilon)=H(x),
$$

где $R(x)$ - функция из (2.4), а $H(x)$ задается формулой

$$
H(x)= \begin{cases}0 & \text { при } x<0, \\ b & \text { при } x>0 .\end{cases}
$$

Равенства (4.2), в свою очередь, позволяют перейти от (4.1) к рассмотрению предельного релейного уравнения с запаздываниями

$$
\dot{x}=R(x(t-h))-H(x(t-1)) .
$$

Как и в случае уравнения (2.4), понятие решения уравнения (4.3) определим конструктивно. С этой целью зафиксируем произвольно натуральное $n$ и предположим, что параметры $h, a, b$ из (1.48)-(1.50) удовлетворяют условиям

$$
\begin{aligned}
\frac{1}{(n+1)(2+a+1 / a)}<h<\frac{1}{n(2+a+1 / a)+2+1 / a}, \\
b>1+a .
\end{aligned}
$$

Далее зафиксируем некоторое достаточно малое $\sigma_{0}>0$ (оценка сверху на этот параметр будет уточнена в последующем), рассмотрим множество функций вида (2.6) и обозначим через $x_{\varphi}(t), t \geqslant-\sigma_{0}$, решение уравнения (4.3) с произвольной начальной функцией (2.6).

При интегрировании уравнения (4.3) следует иметь в виду, что его правая часть представляет собой кусочно постоянную функцию и меняется лишь тогда, когда $x(t-h)$ или $x(t-1)$ меняет знак. В частности, при $-\sigma_{0} \leqslant t \leqslant-\sigma_{0}+h$ имеем одновременно $\varphi(t-h)<0$ и $\varphi(t-1)<0$. Поэтому на указанном промежутке времени согласно $(4.3),(2.6)$ функция $x_{\varphi}(t)$ является решением задачи Коши $\dot{x}=1, x\left(-\sigma_{0}\right)=-\sigma_{0}$, а значит, задается равенством вида (2.7). Ясно также, что формула (2.7) сохраняется до тех пор, пока $x_{\varphi}(t-h)<0$ и $x_{\varphi}(t-1)<0$. Таким образом, она заведомо справедлива на отрезке времени $-\sigma_{0} \leqslant t \leqslant 0$. 
При $0 \leqslant t<1$ в силу уже проделанных построений имеем $x_{\varphi}(t-1)<0$ и, следовательно, $H\left(x_{\varphi}(t-1)\right)=0$. Поэтому на данном промежутке времени интересующее нас решение $x_{\varphi}(t)$ удовлетворяет вспомогательному уравнению

$$
\dot{x}=R(x(t-h)) .
$$

Что же касается уравнения (4.6), то при $h=1$ его свойства были изучены в п. 2.1. Из проделанных там построений следует, что любое решение $x(t)$ этого уравнения такое, что $x(t)<0$ при $-h \leqslant t<0, x(0)=0$, совпадает при всех $t \geqslant 0$ с аналогичной (2.11) периодической функцией

$$
x_{0}(t)=\left\{\begin{array}{ll}
t & \text { при } 0 \leqslant t \leqslant h, \\
h-a(t-h) & \text { при } h \leqslant t \leqslant t_{0}+h, \\
-a h+t-t_{0}-h & \text { при } t_{0}+h \leqslant t \leqslant T_{0},
\end{array} \quad x_{0}\left(t+T_{0}\right) \equiv x_{0}(t),\right.
$$

где теперь (в отличие от разделов 2,3$)$ величины $t_{0}, T_{0}$ задаются равенствами $t_{0}=h(1+1 / a), T_{0}=h(2+a+1 / a)$. График функции (4.7) при $a=2, h=1 / 26$ представлен на рис. 9.

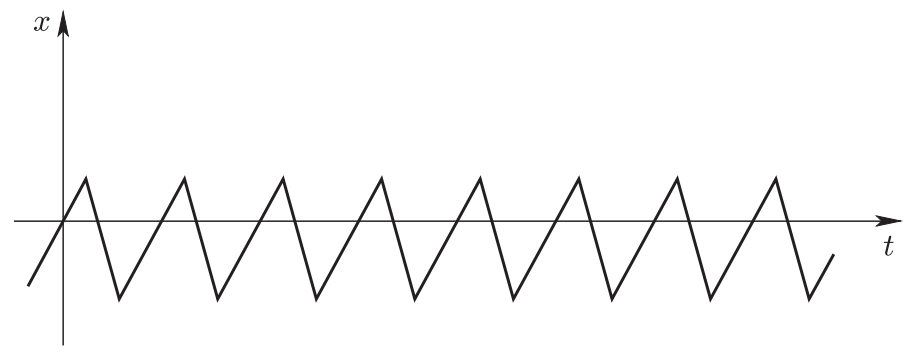

Рис. 9

Возвращаясь к исходному уравнению (4.3) и принимая во внимание все вышесказанное, приходим к равенству

$$
x_{\varphi}(t)=x_{0}(t), \quad 0 \leqslant t \leqslant 1 .
$$

Для последующего анализа нам потребуется специальная функция $y_{0}(t)$, являющаяся решением задачи Коши

$$
\dot{x}=1-H\left(x_{0}(t)\right),\left.\quad x\right|_{t=0}=0 .
$$

Привлекая формулы (4.7), нетрудно увидеть, что при $t \geqslant 0$ она задается соотношениями

$$
y_{0}(t)= \begin{cases}-(b-1) t & \text { при } 0 \leqslant t \leqslant t_{0}, \\ t-b t_{0} & \text { при } t_{0} \leqslant t \leqslant T_{0}, \\ (k-1)\left(T_{0}-b t_{0}\right) & \\ \quad+y_{0}\left(t-(k-1) T_{0}\right) & \text { при }(k-1) T_{0} \leqslant t \leqslant k T_{0}, k \in \mathbb{N}, k \geqslant 2 .\end{cases}
$$


Перейдем к рассмотрению очередного отрезка времени $1 \leqslant t \leqslant 1+h$ и заметим, что неравенства (4.4) обеспечивают принадлежность момента времени $t=1$ интервалу $\left(n T_{0}+t_{0}+h,(n+1) T_{0}\right)$. Тем самым, в силу (4.7), (4.8) имеем $x_{\varphi}(t-h)<0$ при $t \in[1,1+h]$, и, следовательно, на указанном отрезке функция $x_{\varphi}(t)$ является решением аналогичной (4.9) задачи Коши

$$
\dot{x}=1-H\left(x_{0}(t-1)\right),\left.\quad x\right|_{t=1}=x_{0}(1) .
$$

А отсюда заключаем, что при $1 \leqslant t \leqslant 1+h$ справедлива формула

$$
x_{\varphi}(t)=x_{0}(1)+y_{0}(t-1) .
$$

На следующем этапе обратим внимание, что при априорном условии

$$
x_{\varphi}(t-h)<0
$$

равенство (4.11) сохраняется на отрезке $1 \leqslant t \leqslant 2$. Но это условие действительно выполняется, поскольку согласно (4.4), (4.5), (4.7), (4.10)

$$
\begin{gathered}
x_{0}(1)=x_{0}\left(1-n T_{0}\right)=1-(n+1) T_{0}<0, \\
y_{0}(t-1)<0 \quad \text { при всех } t>1 .
\end{gathered}
$$

Таким образом, при $1 \leqslant t \leqslant 2$ соотношение (4.11) обретает законную силу.

При рассмотрении значений $t \geqslant 2$ будем предполагать, что наряду с (4.12) имеет место априорная оценка

$$
x_{\varphi}(t-1)<0 .
$$

В этом случае интересующее нас решение $x_{\varphi}(t)$ определяется из задачи Коши $\dot{x}=1,\left.x\right|_{t=2}=x_{0}(1)+y_{0}(1)$, а значит, задается вытекающей из $(4.10),(4.13)$ формулой

$$
x_{\varphi}(t)=t-T_{*}, \quad T_{*}=(n+1)\left(T_{0}+b t_{0}\right) .
$$

Остается добавить, что согласно (4.11), (4.15) априорные требования (4.12), (4.14) заведомо выполняются на промежутке $2 \leqslant t \leqslant T_{*}$, длина которого в силу неравенства $T_{*}-2>0$ (являющегося следствием оценок (4.4), (4.5)) положительна.

Распорядимся теперь свободным параметром $\sigma_{0}$ (см. (2.6)). Из проделанных выше построений следует, что при условии

$$
\sigma_{0}<T_{0}-t_{0}+(n+1) b t_{0}-1,
$$

которое всюду ниже считаем выполненным, функция $x_{\varphi}\left(t+T_{*}\right)$, рассматриваемая на отрезке $-1-\sigma_{0} \leqslant t \leqslant-\sigma_{0}$, принадлежит введенному выше множеству (2.6). А это значит, что на промежутках $k T_{*}-\sigma_{0} \leqslant t \leqslant(k+1) T_{*}-\sigma_{0}$, $k=1,2, \ldots$, весь описанный выше процесс построения $x_{\varphi}(t)$ повторяется снова и снова. Следовательно, при всех $t \geqslant-\sigma_{0}$ каждое решение $x_{\varphi}(t)$ с начальным условием (2.6) совпадает с одной и той же $T_{*}$-периодической функцией $x_{*}(t)$, задаваемой равенствами

$$
x_{*}(t)=\left\{\begin{array}{ll}
x_{0}(t) & \text { при } 0 \leqslant t \leqslant 1, \\
x_{0}(1)+y_{0}(t-1) & \text { при } 1 \leqslant t \leqslant 2, \\
t-T_{*} & \text { при } 2 \leqslant t \leqslant T_{*},
\end{array} \quad x_{*}\left(t+T_{*}\right) \equiv x_{*}(t) .\right.
$$




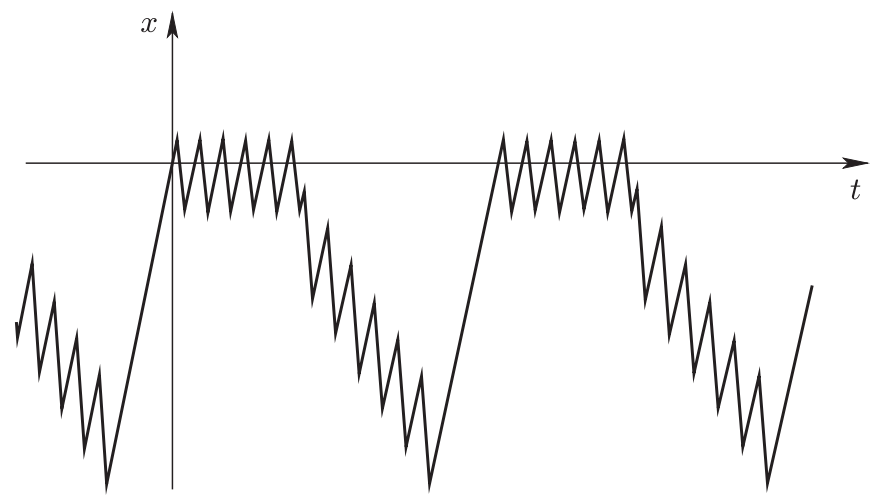

Рис. 10

График этой функции при $a=2, b=4, h=1 / 26$ показан на рис. 10 (при выбранных значениях параметров неравенства (4.4) выполняются для $n=5)$.

Как и в п. 2.1, отдельно остановимся на вопросе о связи между периодическими решениями уравнений (4.1) и (4.3). Имеет место следующее утверждение, являющееся аналогом теоремы 2.1 .

Теорема 4.1. Найдется такое достаточно малое $\varepsilon_{0}>0$, что при всех $0<\varepsilon \leqslant \varepsilon_{0}$ уравнение (4.1) имеет единственный экспоненииально орбитально устойчивый цикл $x_{*}(t, \varepsilon), x_{*}\left(-\sigma_{0}, \varepsilon\right) \equiv-\sigma_{0}$, периода $T_{*}(\varepsilon)$, удовлетворяющий асимптотическим равенствам

$$
\max _{0 \leqslant t \leqslant T_{*}(\varepsilon)}\left|x_{*}(t, \varepsilon)-x_{*}(t)\right|=O(\varepsilon), \quad T_{*}(\varepsilon)=T_{*}+O(\varepsilon), \quad \varepsilon \rightarrow 0 .
$$

Доказательство сформулированной теоремы опирается на некоторые дополнительные конструкции, подобные описанным в п. 2.1. А именно, как и ранее, зафиксируем постоянные $q_{1}>\sigma_{0}, q_{2} \in\left(0, \sigma_{0}\right)$ и обозначим через $S\left(\sigma_{0}, q_{1}, q_{2}\right) \subset$ $C\left[-1-\sigma_{0},-\sigma_{0}\right]$ множество начальных функций $\varphi(t)$, удовлетворяющих требованиям (2.13). Далее, рассмотрим решение $x=x_{\varphi}(t, \varepsilon), t \geqslant-\sigma_{0}$, уравнения (4.1) с начальным условием $\varphi(t) \in S\left(\sigma_{0}, q_{1}, q_{2}\right)$, заданным при $-1-\sigma_{0} \leqslant t \leqslant-\sigma_{0}$, а через $t=T_{\varphi}$ обозначим положительный корень уравнения $x_{\varphi}\left(t-\sigma_{0}, \varepsilon\right)=-\sigma_{0}$ с номером $2 n+2$ (в предположении, что это уравнение имеет на полуоси $t>0$ не менее $2 n+2$ корней, занумерованных в порядке возрастания). И наконец, зададим оператор последования Пуанкаре $\Pi_{\varepsilon}: S\left(\sigma_{0}, q_{1}, q_{2}\right) \rightarrow C\left[-1-\sigma_{0},-\sigma_{0}\right]$ посредством равенства вида (2.14). В процессе обоснования теоремы 4.1 устанавливается, что при соответствующем выборе параметров $\sigma_{0}, q_{1}, q_{2}$ этот оператор переводит множество $S\left(\sigma_{0}, q_{1}, q_{2}\right)$ в себя и является сжимающим.

Подробная реализация описанного плана действий содержится в статье [51] и представляет собой технически усложненный вариант доказательства теоремы 2.1. Поэтому соответствующие выкладки опустим.

Возвращаясь к исходному уравнению (1.48), отметим, что его релаксационный цикл

$$
u_{*}(t, \lambda)=\left.\exp \left(\frac{x_{*}(t, \varepsilon)}{\varepsilon}\right)\right|_{\varepsilon=1 / \lambda}
$$


обладает требуемыми асимптотическими характеристиками, т. е. является bursting-циклом. Действительно, на отрезке времени $0 \leqslant t \leqslant T_{*}(\lambda)$ цикл (4.19) допускает $n+1$ подряд идущих асимптотически высоких (порядка $\exp (\lambda h)$ ) всплеска. Этим всплескам соответствуют интервалы $k T_{0}<t<t_{0}+k T_{0}$, $k=0,1, \ldots, n$, положительности функции (4.17). Если же момент времени $t$ фиксирован и принадлежит множеству $\left[0, T_{*}\right) \backslash \bigcup_{k=0}^{n}\left[k T_{0}, t_{0}+k T_{0}\right]$, то при данном $t$ функция $u_{*}(t, \lambda)$ имеет порядок $\exp (-\lambda q), q=$ const $>0$.

Следует также отметить, что при уменьшении запаздывания $h$ для справедливости теоремы 4.1 необходимо надлежащим образом увеличивать параметр $\lambda$ (связано это с тем, что в рамках нашей асимптотической теории должно выполняться условие $\lambda h \gg 1$ ). Если же, напротив, $h=h_{0} / \lambda, h_{0}=$ const $>0$, то ситуация принципиально меняется. Например, в этом случае может оказаться устойчивым состояние равновесия $u=u_{0}>0$ уравнения (1.48) (существование такого состояния равновесия обеспечивают условия (1.49), а при вполне естественных требованиях " $f^{\prime}(u)<0, g^{\prime}(u)>0$ для любого $u \in \mathbb{R}_{+}$" оно единственно). Кроме того, используя так называемый метод квазинормальных форм (см. [52], [53]), можно показать, что при некоторых дополнительных предположениях из состояния равновесия $u=u_{0}$ бифурцирует любое наперед заданное число сосуществующих устойчивых циклов, т. е. реализуется феномен буферности. Соответствующий анализ излагается п. 4.2.

В заключение добавим, что условия (4.4) на $h$ автоматически означают, что $h<1$. Однако аналог теоремы 4.1 справедлив и в случае $h>1$. Для того чтобы убедиться в этом, обратимся к уравнению (1.47) и в предположении $h_{1}>h_{2}$ выполним в нем нормировку времени $t / h_{1} \rightarrow t$. В результате вместо (1.48) получим уравнение

$$
\dot{u}=\lambda[f(u(t-1))-g(u(t-h))] u
$$

где $h=h_{2} / h_{1}<1$.

Далее, рассмотрим отвечающее уравнению (4.20) релейное уравнение

$$
\dot{x}=R(x(t-1))-H(x(t-h))
$$

и заметим, что при условии $b>1$ оно преобразуется к виду (4.3). Действительно, полагая $\widetilde{a}=b-1, \widetilde{b}=a+1$,

$$
\widetilde{R}(x)=\left\{\begin{array}{ll}
1 & \text { при } x<0, \\
-\widetilde{a} & \text { при } x>0,
\end{array} \quad \widetilde{H}(x)= \begin{cases}0 & \text { при } x<0, \\
\widetilde{b} & \text { при } x>0,\end{cases}\right.
$$

приходим к выводу, что уравнение (4.21) записывается в требуемой форме

$$
\dot{x}=\widetilde{R}(x(t-h))-\widetilde{H}(x(t-1)) .
$$

А это, в свою очередь, означает, что если параметры $\widetilde{a}, \widetilde{b}, h$ удовлетворяют аналогичным (4.4), (4.5) неравенствам, то для уравнения (4.20) сохраняется утверждение теоремы 4.1 . 
4.2. Явление буферности. В данном пункте исследуем вопрос о локальных аттракторах уравнения (1.48), расположенных в малой окрестности состояния равновесия. В связи с этим сначала приведем это уравнение к некоторому специальному виду, удобному для соответствующего анализа, и попутно уточним ограничения на функции $f(u), g(u)$.

В первую очередь положим в (1.48)

$$
f(u)=(a+1) \tilde{f}(u)-a, \quad g(u)=b \widetilde{g}(u), \quad h=\varepsilon \widetilde{h}, \quad \varepsilon=\frac{1}{\lambda},
$$

а затем вернемся к прежним обозначениям, т. е. заменим снова $\widetilde{f}(u), \widetilde{g}(u), \widetilde{h}$ на $f(u), g(u), h$. В результате приходим к дифференциально-разностному уравнению вида

$$
\varepsilon \dot{u}=[(a+1) f(u(t-\varepsilon h))-a-b g(u(t-1))] u,
$$

где в силу $(1.49),(4.22)$ новые функции $f(u), g(u)$ удовлетворяют равенствам

$$
f(0)=1, \quad g(0)=0, \quad \lim _{u \rightarrow+\infty} f(u)=0, \quad \lim _{u \rightarrow+\infty} g(u)=1 .
$$

Кроме того, в дальнейшем считаем, что $f(u), g(u) \in C^{\infty}\left(\mathbb{R}_{+}\right)$и выполняются следующие три дополнительных ограничения.

УСловие 4.1. Справедливы неравенства $f^{\prime}(u)<0, g^{\prime}(u)>0$ для всех $u \in \mathbb{R}_{+}$.

Прежде чем формулировать второе условие, обратим внимание, что условие 4.1 и свойства (4.24) в совокупности обеспечивают существование и единственность решения $u=u_{0}(a, b)>0$ уравнения $(a+1) f(u)-a-b g(u)=0$. Далее, считая параметр $a>0$ фиксированным, введем в рассмотрение функцию $\psi(b)$, $b \in \mathbb{R}_{+}$, задаваемую соотношением

$$
\psi(b)=\left.(a+1) f^{\prime}(u)\right|_{u=u_{0}(a, b)}+\left.b g^{\prime}(u)\right|_{u=u_{0}(a, b)} .
$$

УСловие 4.2. Предполагаем, что уравнение $\psi(b)=0$ имеет на полуоси $b \in \mathbb{R}_{+}$единственное решение $b=b_{*}>0 u \psi^{\prime}\left(b_{*}\right)>0$.

Остановимся на связи между условиями 4.1 и 4.2. Несложный подсчет показывает, что функция $u_{0}(a, b)$ по переменной $b$ обладает свойствами

$$
u_{0}(a, 0)=u_{0,0}>0, \quad u_{0}(a, b)=\frac{1}{g^{\prime}(0) b}+O\left(\frac{1}{b^{2}}\right) \quad \text { при } b \rightarrow+\infty,
$$

где $u_{0,0}$ - корень уравнения $f(u)=a /(a+1)$, который в силу условия 4.1 определяется однозначно. Подставляя затем соотношения (4.26) в (4.25), приходим к выводу, что $\psi(0)<0, \lim _{b \rightarrow+\infty} \psi(b)=+\infty$. Таким образом, условие 4.1 гарантирует существование у функции (4.25) хотя бы одного корня $b=b_{*}>0$, $\psi^{\prime}\left(b_{*}\right) \geqslant 0$, а условие 4.2 содержит вполне естественные требования о единственности этого корня и о выполнении неравенства $\psi^{\prime}\left(b_{*}\right)>0$, характеризующего некоторую общность положения. 
Для формулировки последнего условия нам потребуются функции

$$
\begin{aligned}
& V_{1}(v, b)=\left.\frac{1}{2 f^{\prime}(u) u}\left[f(u(1+v))-f(u)-f^{\prime}(u) u v\right]\right|_{u=u_{0}(a, b)} \\
& V_{2}(v, b)=-\left.\frac{b}{2(a+1) f^{\prime}(u) u}\left[g(u(1+v))-g(u)-g^{\prime}(u) u v\right]\right|_{u=u_{0}(a, b)}
\end{aligned}
$$

Нетрудно убедиться, что их тейлоровские разложения в точке $v=0$ начинаются с квадратичных слагаемых. В частности, справедливы равенства

$$
V_{1}\left(v, b_{*}\right)=c_{1} v^{2}+c_{2} v^{3}+O\left(v^{4}\right), \quad V_{2}\left(v, b_{*}\right)=d_{1} v^{2}+d_{2} v^{3}+O\left(v^{4}\right) .
$$

Условие 4.3. Считаем, что $d \stackrel{\text { def }}{=} 4\left(c_{1}^{2}-d_{1}^{2}\right)+2\left(d_{2}-c_{2}\right)<0$.

Все перечисленные условия выполняются, например, для функций

$$
f(u)=\frac{1}{1+u}, \quad g(u)=1-\frac{1}{\sqrt{1+u}} .
$$

В симметричном же случае, когда $g(u)=1-f(u)$, с необходимостью имеем $d=0$.

При сформулированных ограничениях поставим вопрос об аттракторах уравнения (4.23), бифурцирующих из состояния равновесия $u=u_{0}(a, b)$ при изменении параметров $b, h$. В связи с этим распорядимся выбором указанных параметров таким образом, чтобы в задаче об устойчивости интересующего нас положения равновесия реализовывался случай, близкий к максимальному вырождению. Подчеркнем, что именно в окрестности максимальной особенности соответствующий локальный анализ может дать наиболее полную информацию об автоколебательных режимах исходного уравнения.

Остановимся сначала на выборе параметра $b$. Будем считать, что он меняется в малой окрестности критического значения $b=b_{*}$, о котором идет речь в условии 4.2. Точнее говоря, положим

$$
b=b_{*}+\alpha(\mu), \quad|\mu| \ll 1,
$$

где $\mu$ - вспомогательный малый параметр, а функция $\alpha(\mu), \alpha(0)=0$, определяется из уравнения

$$
\left.\psi(\alpha, \mu) \stackrel{\text { def }}{=} \frac{(1+a) f^{\prime}(u)}{\left(b_{*}+\alpha\right) g^{\prime}(u)}\right|_{u=u_{0}\left(a, b_{*}+\alpha\right)}+\frac{1-2 \mu}{1+2 \mu}=0 .
$$

Остается заметить, что в силу условия 4.2

$$
\psi(0,0)=0, \quad \psi_{\alpha}^{\prime}(0,0)=\frac{\psi^{\prime}\left(b_{*}\right)}{b_{*} g^{\prime}\left(u_{0}\left(a, b_{*}\right)\right)}>0
$$

и, следовательно, к уравнению $(4.32)$ в точке $(\alpha, \mu)=(0,0)$ применима теорема о неявной функции по переменной $\alpha$. 
Опишем теперь способ выбора параметра $h$. В связи с этим подставим в уравнение (4.23) соотношение (4.31) и перейдем к новой переменной $v$, полагая $u=u_{*}(\mu)(1+v)$, где $u_{*}(\mu)=u_{0}\left(a, b_{*}+\alpha(\mu)\right)$. В результате после нормировок

$$
\frac{\varepsilon}{\varkappa_{*}(\mu)} \rightarrow \varepsilon, \quad h \varkappa_{*}(\mu) \rightarrow h, \quad \varkappa_{*}(\mu) \stackrel{\text { def }}{=}-\frac{2(1+a) f^{\prime}\left(u_{*}(\mu)\right) u_{*}(\mu)}{1-2 \mu}>0
$$

рассматриваемое уравнение преобразуется к более удобному для последующего анализа виду

$$
\begin{aligned}
\varepsilon \dot{v}=- & {\left[\left(\frac{1}{2}-\mu\right) v(t-\varepsilon h)+\left(\frac{1}{2}+\mu\right) v(t-1)\right.} \\
& \left.+\Delta_{1}(v(t-\varepsilon h), \mu)+\Delta_{2}(v(t-1), \mu)\right](1+v),
\end{aligned}
$$

где $\Delta_{j}=(1-2 \mu) V_{j}\left(v, b_{*}+\alpha(\mu)\right), j=1,2$ (см. (4.27), (4.28)).

Введем в рассмотрение характеристическое уравнение

$$
\varepsilon \lambda+\left(\frac{1}{2}-\mu\right) \exp (-\varepsilon h \lambda)+\left(\frac{1}{2}+\mu\right) \exp (-\lambda)=0,
$$

отвечающее нулевому состоянию равновесия уравнения (4.33). Расположение его корней изучено в [51], где показано, что в случае $h<\pi$ все корни распадаются на две группы. K первой группе отнесем некритические корни, которые находятся в левой комплексной полуплоскости $\{\lambda: \operatorname{Re} \lambda<0\}$ и не приближаются к мнимой оси при $\varepsilon, \mu \rightarrow 0$. Во вторую группу объединим все оставшиеся корни $\lambda_{n}(\varepsilon, \mu), \bar{\lambda}_{n}(\varepsilon, \mu), n \in \mathbb{N}$, этого уравнения, являющиеся комплексными и при $\varepsilon=\mu=0$ обращающиеся в соответствующие корни $\lambda_{n}=i \omega_{n}, \omega_{n}=\pi(2 n-1)$, $n \in \mathbb{N}$, уравнения $\exp (-\lambda)=-1$. Как установлено в статье [53], эти корни допускают равномерные по $n$ асимптотические представления

$$
\begin{gathered}
\operatorname{Re} \lambda_{n}(\varepsilon, \mu)=\left.\delta(z, \mu)\right|_{z=\varepsilon \omega_{n}}+O(\varepsilon), \\
\delta(z, \mu)=\ln (1+2 \mu)-\frac{1}{2} \ln \left[(1-2 \mu)^{2}-4 z(1-2 \mu) \sin (h z)+4 z^{2}\right] .
\end{gathered}
$$

Добавим еще, что при условии $h<1$ справедливы неравенства $\delta(z, 0)<0$ для всех $z>0$ и $\delta_{z z}^{\prime \prime}(0,0)=-4(1-h)<0$. Следовательно, в этом случае при $\varepsilon, \mu \rightarrow 0$ и $n \rightarrow \infty$ корни $\lambda_{n}(\varepsilon, \mu)$ заведомо не могут иметь положительных действительных частей порядка единицы. Если же, напротив, $h>1$, то $\delta(z, 0)>0$ на некотором промежутке вида $0<z \leqslant z_{0}$, а значит, у уравнения (4.34) с необходимостью существуют корни в полуплоскости $\operatorname{Re} \lambda \geqslant c$, где $c>0$ - не зависящая от $\varepsilon$ и $\mu$ постоянная. В этом случае поставленная выше бифуркационная проблема очевидным образом теряет смысл.

Из всего вышесказанного следует, что интересующая нас ситуация, когда в задаче об устойчивости нулевого решения уравнения (4.33) имеет место максимальное вырождение, реализуется при $h=1+\nu,|\nu| \ll 1$. Точнее говоря, вопрос о бифурцирующих из нуля автоколебаниях этого уравнения будем изучать при дополнительных условиях

$$
\mu=\mu_{0} \varepsilon^{4}, \quad h=1+\nu_{0} \varepsilon^{2}, \quad \mu_{0}, \nu_{0}=\text { const } \in \mathbb{R},
$$

смысл которых прояснится в последующем. 
Проблему аттракторов уравнения (4.33), расположенных в малой окрестности нуля фазового пространства $C[-1,0]$, позволяет решить так называемый метод квазинормальных форм. Упомянутый метод был разработан Ю. С. Колесовым в начале 80-х годов прошлого века для исследования автоколебаний в случае, близком к бесконечномерному вырождению. Общая идея этого метода заключается в построении формальной нормальной формы Пуанкаре-Дюлака в окрестности положения равновесия и в последующем анализе получившейся счетной системы обыкновенных дифференциальных уравнений (квазинормальной формы). Что же касается алгоритмических аспектов процедуры нормализации, то они весьма многообразны (см. монографии [38], [39], [54], где рассмотрены различные примеры применения метода квазинормальных форм и приведено его обоснование в некоторых модельных ситуациях).

В нашем случае алгоритмическая часть метода квазинормальных форм состоит в следующем. При условиях (4.35) главную асимптотику возможных автоколебательных режимов уравнения (4.33) будем искать в виде

$$
v=\sum_{k=1}^{5} \varepsilon^{k+1} v_{k}(\tau, \theta, s), \quad \tau=\left(1+\sum_{k=1}^{4} \varepsilon^{k} \sigma_{k}\right) t,
$$

где $\theta=\varepsilon^{3} t, s=\varepsilon^{4} t, v_{k}(\tau, \theta, s)$ - подлежащие определению 2-периодические по $\tau$ функции, а постоянные $\sigma_{k}$ заданы равенствами

$$
\sigma_{1}=-1, \quad \sigma_{2}=1, \quad \sigma_{3}=\nu_{0}-1, \quad \sigma_{4}=-2 \nu_{0}+1 .
$$

Подставляя соотношения (4.36), (4.37) в (4.33) и приравнивая коэффициенты при одинаковых степенях $\varepsilon$ в левой и правой частях получившегося равенства, для нахождения функций $v_{k}$ получаем рекуррентную последовательность линейных неоднородных разностных уравнений

$$
\frac{1}{2}\left[v_{k}(\tau, \theta, s)+v_{k}(\tau-1, \theta, s)\right]=\mathscr{F}_{k}^{(1)}(\tau, \theta, s)+\mathscr{F}_{k}^{(2)}(\tau, \theta, s),
$$

где $\mathscr{F}_{k}^{(j)}(\tau+1, \theta, s) \equiv(-1)^{j+1} \mathscr{F}_{k}^{(j)}(\tau, \theta, s), j=1,2$. Условие же разрешимости каждого уравнения (4.38) в требуемом классе функций имеет вид

$$
\mathscr{F}_{k}^{(2)}(\tau, \theta, s) \equiv 0,
$$

и если оно выполняется, то само решение $v_{k}$ задается формулой

$$
v_{k}=\mathscr{F}_{k}^{(1)}(\tau, \theta, s)+v_{k}^{0}(\tau, \theta, s), \quad v_{k}^{0}(\tau+1, \theta, s) \equiv-v_{k}^{0}(\tau, \theta, s),
$$

где $v_{k}^{0}$ - некоторая произвольная функция.

Первые три шага описанной процедуры приводят к равенствам

$$
v_{1}=v_{1}^{0}(\tau, \theta, s), \quad v_{2}=v_{2}^{0}(\tau, \theta, s), \quad v_{3}=-\left(c_{1}+d_{1}\right)\left(v_{1}^{0}\right)^{2}+v_{3}^{0}(\tau, \theta, s),
$$

где $c_{1}, d_{1}-$ коэффициенты тейлоровских разложений (4.29). Подчеркнем, что при $k=1$ разрешимость уравнения (4.38) очевидна (в этом случае функции $\mathscr{F}_{1}^{(1)}, \mathscr{F}_{1}^{(2)}$ равны нулю), а при $k=2,3$ справедливость условий (4.39) обеспечивается специальным выбором постоянных $\sigma_{1}, \sigma_{2}$ (см. (4.37)). 
На четвертом шаге алгоритма с учетом известной информации (4.37), (4.40) приходим к равенству $\mathscr{F}_{4}^{(2)}=\frac{1}{6}\left(\frac{\partial^{3} v_{1}^{0}}{\partial \tau^{3}}-3 \frac{\partial v_{1}^{0}}{\partial \theta}\right)$. А отсюда и из (4.39) заключаем, что функция $v_{1}^{0}$ должна удовлетворять краевой задаче

$$
\frac{\partial v_{1}^{0}}{\partial \theta}=\frac{1}{3} \frac{\partial^{3} v_{1}^{0}}{\partial \tau^{3}}, \quad v_{1}^{0}(\tau+1, \theta, s) \equiv-v_{1}^{0}(\tau, \theta, s),
$$

где $\tau$ играет роль пространственной переменной, $\theta$ - время, а переменная $s$ считается параметром. Тем самым, для $v_{1}^{0}$ получаем представление

$$
v_{1}^{0}=\sum_{n=1}^{\infty} \xi_{n}(s) \exp \left[i \omega_{n} \tau-\frac{i \omega_{n}^{3} \theta}{3}\right]+\bar{\xi}_{n}(s) \exp \left[-i \omega_{n} \tau+\frac{i \omega_{n}^{3} \theta}{3}\right],
$$

где $\xi_{n}(s), n \geqslant 1,-$ пока произвольные комплексные амплитуды.

Заключительный этап связан с рассмотрением уравнений (4.38), (4.39) при $k=5$. Соответствующий подсчет показывает, что в данном случае из (4.39) для $v_{2}^{0}$ получается аналогичная (4.41) линейная неоднородная краевая задача

$$
\frac{\partial v_{2}^{0}}{\partial \theta}-\frac{1}{3} \frac{\partial^{3} v_{2}^{0}}{\partial \tau^{3}}=\Phi(\tau, \theta, s), \quad v_{2}^{0}(\tau+1, \theta, s) \equiv-v_{2}^{0}(\tau, \theta, s),
$$

где

$$
\Phi=d\left(v_{1}^{0}\right)^{3}+4 \mu_{0} v_{1}^{0}-\frac{\partial v_{1}^{0}}{\partial s}-2 \nu_{0} \frac{\partial^{2} v_{1}^{0}}{\partial \tau^{2}}-\frac{\partial^{3} v_{1}^{0}}{\partial \tau^{3}}-\frac{\partial^{2} v_{1}^{0}}{\partial \tau \partial \theta},
$$

a $d$ - постоянная из условия 4.3. Заметим, далее, что в силу (4.42) неоднородность (4.44) представляет собой формальный тригонометрический ряд переменных $\tau, \theta$. В таком же виде будем искать и $v_{2}^{0}$. Для этого, однако, необходимо и достаточно, чтобы в ряде для $\Phi$ отсутствовали гармоники вида $\exp ( \pm i y)$, $y=\omega_{n} \tau-\omega_{n}^{3} \theta / 3, n \in \mathbb{N}$. Добиться же равенства нулю коэффициентов при данных гармониках удается за счет подходящего выбора имеющихся в запасе комплексных амплитуд $\xi_{n}(s), n \geqslant 1$. Этот путь приводит к счетной системе обыкновенных дифференциальных уравнений для $\xi_{n}$ :

$$
\frac{d \xi_{n}}{d s}=\left(\gamma_{n}+i \omega_{n}^{3}\right) \xi_{n}+d\left(3\left|\xi_{n}\right|^{2}+6 \sum_{\substack{m=1 \\ m \neq n}}^{\infty}\left|\xi_{m}\right|^{2}\right) \xi_{n}, \quad n \geqslant 1
$$

где $\gamma_{n}=4 \mu_{0}+2 \nu_{0} \omega_{n}^{2}-\omega_{n}^{4} / 3$.

Система (4.45) и является интересующей нас квазинормальной формой исходного уравнения (4.33). Для формулировки основного результата перейдем от (4.45) к соответствующей амплитудной системе для $\eta_{n}=3\left|\xi_{n}\right|^{2}, n \geqslant 1$, которая после нормировки времени $2 s \rightarrow s$ примет вид

$$
\frac{d \eta_{n}}{d s}=\gamma_{n} \eta_{n}+d\left(\eta_{n}+2 \sum_{m \neq n} \eta_{m}\right) \eta_{n}, \quad n \geqslant 1
$$

Будем рассматривать (4.46) как динамическую систему в фазовом пространстве $l=\left\{\eta=\left(\eta_{1}, \ldots, \eta_{n}, \ldots\right):\|\eta\|=\sum_{n=1}^{\infty}\left|\eta_{n}\right|<\infty\right\}$. Справедливо следующее утверждение, аналоги которого установлены в [38], [39], [54]. 
Теорема 4.2. Любому фиксированному экспоненциалъно устойчивому или дихотомичному состоянию равновесия системы (4.46) с конечным числом ненулевых координат $\eta_{n_{j}}>0, j=1, \ldots, p$, в исходном уравнении (4.33) при условиях (4.35) и при всех достаточно малых $\varepsilon>0$ соответствует р-мерный инвариантный тор той же устойчивости. Главная асимптотика автоколебаний на этом торе задается соотношениями (4.36), (4.40), (4.42), в которых следует положить

$$
\xi_{n_{j}}(s)=\sqrt{\frac{\eta_{n_{j}}}{3}} \exp \left(i \omega_{n_{j}}^{3} s\right), \quad j=1, \ldots, p ; \quad \xi_{n}(s)=0 \quad \text { npu } n \neq n_{j} .
$$

Перейдем к вопросу об аттракторах уравнения (4.33), доставляемых теоремой 4.2. В связи с этим обратим внимание, что любое состояние равновесия системы (4.46) из инвариантного конуса $K=\left\{\eta \in l: \eta_{n} \geqslant 0 \forall n \in \mathbb{N}\right\}$, имеющее $p \geqslant 2$ ненулевых координат, оказывается неустойчивым. Устойчивыми же могут быть лишь ее состояния равновесия вида

$$
O_{n}=\left\{\eta \in l: \eta_{n}=-\frac{\gamma_{n}}{d}, \eta_{m}=0 \text { при } m \neq n\right\} .
$$

Точнее говоря, в силу неравенства $d<0$ состояние равновесия (4.47) с фиксированным номером $n$ принадлежит конусу $K$ при условии $\gamma_{n}>0$, а при дополнительном требовании $\gamma_{n}>(1 / 2) \max _{m} \geqslant 1 \gamma_{m}$ оказывается устойчивым. Добавим еще, что при согласованном увеличении параметров $\mu_{0}$ и $\nu_{0}$ оба эти условия будут выполняться для номеров $n_{1} \leqslant n \leqslant n_{2}$, где разность $n_{2}-n_{1}$ может быть сделана сколь угодно большой. А отсюда и из теоремы 4.2 вытекает следующее утверждение.

ТЕОРема 4.3. По любому натуральному $n_{0}$ можно так подобрать фигурирующие в (4.35) параметрь $\mu_{0}, \nu_{0}$, что при условиях (4.35) и при всех достаточно малых $\varepsilon>0$ уравнение (4.33) будет иметь не менее $n_{0}$ сосуществующих экспоненииально орбитально устойчивых периодических движений.

Сформулированная теорема свидетельствует о том, что при выполнении условий 4.1-4.3 в исходном уравнении (4.23) наблюдается феномен буферности. Реализуемость этого феномена подтверждает и численный эксперимент, который проводился для уравнения (4.23) с функциями (4.30) при значениях параметров $a=2, b=5.48, \varepsilon=1 / 130, \varepsilon h=0.016$. Точнее говоря, в $(4.23)$ предварительно была выполнена замена $u=\exp (x / \varepsilon)$, а затем получившееся уравнение для $x$ интегрировалось численно методом Рунге-Кутта четвертого порядка точности с постоянным шагом $\Delta t=2 \cdot 10^{-5}$. В результате удалось обнаружить не менее 60 сосуществующих устойчивых циклов.

В заключение добавим, что буферность вполне естественна для нейронных систем, поскольку она отражает ситуацию, когда в неокортексе человеческого мозга различные идеи и концепции соревнуются друг с другом в целях доминирования. Весьма нетривиальным здесь является то обстоятельство, что добиться наличия этого свойства можно не только за счет усложнения системы, т. е. рассмотрения цепочек или решеток связанных осцилляторов вида (4.23). Как оказалось, буферность свойственна даже отдельному нейрону, что свидетельствует о содержательности предложенной нами математической модели. 


\section{5. Заключение}

В настоящей статье мы стремились дать общие представления об идеях и методах, лежащих в основе математического моделирования нейродинамических процессов. Была приведена серия известных базовых математических моделей как отдельного нейрона, так и нейронных сетей, а также представлена наша новая модель функционирования отдельного нейрона (1.48). Полученные математические результаты свидетельствуют о том, что наша модель сочетает в себе внешнюю простоту с возможностью описать такие фундаментальные нейродинамические явления, как bursting-эффект и буферность.

Исторически сложилось так, что основное внимание исследователей было уделено эффекту "bursting behavior". В случае же феномена буферности ситуация оказалась несколько иной. Несмотря на то что данное явление представляет собой один из фундаментальных законов функционирования нелинейных систем из различных областей естествознания [38], [39], оно до сих пор не получило должного освещения в нейродинамической литературе. В то же время достаточно ясно, что буферность весьма актуальна именно для нейронной тематики. Действительно, она отражает конкурентное взаимодействие различных концепций и идей в неокортексе человеческого мозга, а также может быть использована для объяснения механизма работы ассоциативной памяти. Остановимся на последнем чуть более подробно.

Согласно монографии [55] в простейшем случае ассоциативная память работает по схеме, представленной на рис. 11. А именно, имеется некоторая запоминающая среда, реализованная в виде нейронной системы и хранящая в себе блоки информации $R_{n}, n=1,2, \ldots, n_{0}$. Для извлечения из системы конкретного результата $R_{n}$ необходимо на ее вход подать соответствующий символьный ключ $K_{n}$ и некоторую дополнительную информацию $C_{n}$, называемую контекстом.

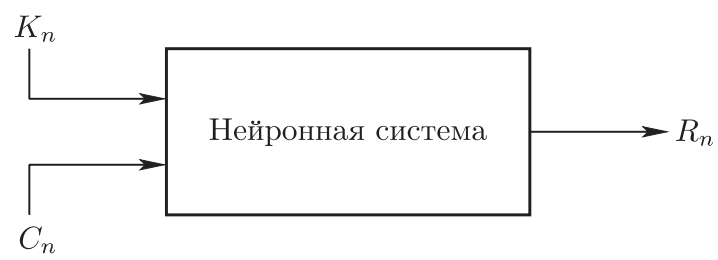

Рис. 11

Предположим теперь, что запоминающая среда описывается отдельным уравнением (1.48) или системой связанных уравнений типа (1.48). Далее, будем считать параметры данной системы выбранными таким образом, что она имеет $n_{0}$ сосуществующих устойчивых циклов, занумерованных индексами $n=$ $1,2, \ldots, n_{0}$. И наконец, допустим, что с каждым из этих циклов связан соответствующий блок информации $R_{n}$.

Работа приведенной математической модели ассоциативной памяти вполне понятна: для того чтобы получить требуемую информацию $R_{n}$, необходимо заставить систему функционировать в устойчивом периодическом режиме с соот- 
ветствующим номером $n$. Добиться желаемого результата удается за счет введения ключа $K_{n}=n$ и дополнительного контекста $C_{n}$. В качестве $C_{n}$ в данном случае уместно взять начальные условия упомянутого периодического режима.

В разделе 4 феномены bursting и буферности были обнаружены в уравнении (1.48) по отдельности: каждый в своем диапазоне параметров. Однако эти явления могут быть реализованы совместно в рамках некой единой модели. Одна из таких моделей имеет вид

$$
\begin{gathered}
\dot{u}_{j}=d\left(u_{j+1}-2 u_{j}+u_{j-1}\right)+\lambda\left[f\left(u_{j}(t-h)\right)-g\left(u_{j}(t-1)\right)\right] u_{j}, \\
j=1, \ldots, m,
\end{gathered}
$$

где $u_{0}=u_{1}, u_{m+1}=u_{m}, d=$ const $>0, \lambda \gg 1$.

Система (5.1) исследовалась в [56], где на нее были распространены аналоги теорем $3.1,3.2$. Попутно было установлено, что при увеличении $m$ и при согласованном стремлении $d$ к 0 и $\lambda \mathrm{K}+\infty$ в системе (5.1) происходит неограниченное накапливание устойчивых bursting-циклов. Термин bursting в данном случае означает, что компоненты $u_{j}, j=1, \ldots, m$, каждого из этих циклов демонстрируют то же самое асимптотическое поведение, что и функция $u_{*}(t, \lambda)$ из $(4.19)$, а именно, допускают на периоде ровно $n$ асимптотически высоких всплесков. Что же касается натурального $n$, то при соответствующем выборе параметров оно может быть любым.

Отдельного упоминания заслуживает следующее обстоятельство. Все приведенные в данной статье результаты остаются в силе при более общих требованиях на функции $f(u), g(u)$ из (1.48). А именно, можно предполагать, что при $u \rightarrow+\infty$ вместо (1.50) выполняются асимптотические равенства

$$
\begin{aligned}
& f(u)=-a+O\left(u^{-\gamma_{1}}\right), \quad u f^{\prime}(u)=O\left(u^{-\gamma_{1}}\right), \quad u^{2} f^{\prime \prime}(u)=O\left(u^{-\gamma_{1}}\right), \\
& g(u)=b+O\left(u^{-\gamma_{2}}\right), \quad u g^{\prime}(u)=O\left(u^{-\gamma_{2}}\right), \quad u^{2} g^{\prime \prime}(u)=O\left(u^{-\gamma_{2}}\right)
\end{aligned}
$$

при произвольно фиксированных $\gamma_{1}, \gamma_{2}>0$.

В заключение остановимся на наиболее актуальных нерешенных проблемах. Представляет несомненный интерес исследование аттракторов в аналогичных (3.73), (3.102) системах

$$
\begin{gathered}
\dot{u}_{j}=d\left(u_{j+1}-u_{j}\right)+\lambda\left[f\left(u_{j}(t-h)\right)-g\left(u_{j}(t-1)\right)\right] u_{j}, \\
j=1, \ldots, m, \quad u_{m+1}=u_{1} ; \\
\dot{u}_{j}=d \sum_{i=1}^{m}\left(u_{i}-u_{j}\right)+\lambda\left[f\left(u_{j}(t-h)\right)-g\left(u_{j}(t-1)\right)\right] u_{j}, \quad j=1, \ldots, m,
\end{gathered}
$$

где, как обычно, $d=$ const $>0, \lambda \gg 1$. Отметим сразу, что для систем $(5.2),(5.3)$ остаются в силе аналоги теорем 3.1, 3.2. Тем самым, проблема их аттракторов сводится к исследованию соответствующих $(m-1)$-мерных отображений, подобных (3.27), но эти отображения пока не изучены.

Еще один интересный нерешенный вопрос связан с системой (3.102). Выше мы ограничились рассмотрением простейших ее периодических решений так называемых режимов двухкластерной синхронизации. Однако эта система допускает и решения типа $s$-кластерной синхронизации, где $2 \leqslant s \leqslant m$. 
Действительно, предположим, что имеет место аналогичное (3.103) разбиение

$$
\{1,2, \ldots, m\}=\Sigma_{1} \cup \Sigma_{2} \cup \cdots \cup \Sigma_{s},
$$

где $\Sigma_{i} \cap \Sigma_{j}=\varnothing$ при $i \neq j$. Далее, пусть $k_{r}$ - количество элементов в множестве $\Sigma_{r}$, причем $k_{1}+\cdots+k_{s}=m$. Тогда у системы (3.102) существуют решения вида

$$
u_{j}=w_{r}(t), \quad j \in \Sigma_{r}, \quad r=1, \ldots, s,
$$

где компоненты $w_{r}(t), r=1, \ldots, s$, попарно различны и удовлетворяют системе

$$
\begin{gathered}
\dot{w}_{r}=d\left(r_{1} w_{1}+r_{2} w_{2}+\cdots+r_{s} w_{s}-m w_{r}\right)+\lambda f\left(w_{r}(t-1)\right) w_{r}, \\
r=1, \ldots, s .
\end{gathered}
$$

Если среди решений (5.4) имеется периодическое, то его уместно назвать периодическим режимом $s$-кластерной синхронизации.

Вопрос о существовании и устойчивости $s$-кластерных периодических режимов системы (3.102) при $s \geqslant 3$ остается открытым. То же самое относится и к $s$-кластерным режимам системы (5.3) при $s \geqslant 2$.

\section{Список литературы}

[1] E. M. Izhikevich, Dynamical systems in neuroscience: the geometry of excitability and bursting, Comput. Neurosci., MIT Press, Cambridge, MA, 2007, xvi+441 pp.

[2] А.Л. Ходжкин, Нервный импульс, Мир, М., 1965, 125 с.; пер. с англ.: A. L. Hodgkin, The conduction of the nervous impulse, Sherrington Lectures, 7, C. C. Thomas, Publisher, Springfield, IL, 1964, 108 pp.

[3] C. Koch, I. Segev, Methods in neuronal modeling. From ions to networks, 2nd ed., Comput. Neurosci., MIT Press, Cambridge, MA, 1998, 687 pp.

[4] A. L. Hodgkin, A. F. Huxley, "A quantitative description of membrane current and its application to conduction and excitation in nerve", J. Physiol., 117:4 (1952), 500-544.

[5] A. L. Hodgkin, A. F. Huxley, "Action potentials recorded from inside a nerve fiber", Nature, 144 (1939), 710-711.

[6] A. L. Hodgkin, "The local electric changes associated with repetitive action in a nonmedullated axon", J. Physiol., 107:2 (1948), 165-181.

[7] D. Hansel, G. Mato, C. Meunier, "Synchrony in excitatory neural networks", Neural Cотр., 7:2 (1995), 307-337.

[8] Е. Ф. Мищенко, Н. Х. Розов, Дифференииальные уравнения с малым параметром и релаксационные колебания, Наука, М., 1975, 247 с.; англ. пер.: Е. F. Mishchenko, N. Kh. Rozov, Differential equations with small parameters and relaxation oscillations, Math. Concepts Methods Sci. Engrg., 13, Plenum Press, New York, 1980, x+228 pp.

[9] R. FitzHugh, "Mathematical models of threshold phenomena in the nerve membrane", Bull. Math. Biophys., 17:4 (1955), 257-278.

[10] R. FitzHugh, "Threshold and plateaus in the Hodgkin-Huxley nerve equations", J. Gen. Physiol., 43 (1960), 867-896.

[11] R. FitzHugh, "Impulses and physiological states in theoretical models of nerve membrane", Biophys. J., 1:6 (1961), 445-466.

[12] J. Nagumo, S. Arimoto, S. Yoshizawa, "An active pulse transmission line simulating nerve axon", Proc. IRE, 50:10 (1962), 2061-2070. 
[13] C. Morris, H. Lecar, "Voltage oscillations in the barnacle giant muscle fiber", Biophys. J., 35:1 (1981), 193-213.

[14] J. L. Hindmarsh, R. M. Rose, "A model of the nerve impulse using two first-order differential equations", Nature, 296 (1982), 162-164.

[15] J. L. Hindmarsh, R. M. Rose, "A model of neuronal bursting using three coupled first order differential equations", Proc. Roy. Soc. London Ser. B, 221 (1984), 87-102.

[16] С. Д. Глызин, А.Ю. Колесов, Н.Х. Розов, “Об одной модификации нейронной модели ФитцХью-Нагумо", Журн. вычисл. матем. и матем. физ., 54:3 (2014), 430-449; S. D. Glyzin, A. Yu. Kolesov, N. Kh. Rozov, "On a modification of the FitzHugh-Nagumo neuron model", Comput. Math. Math. Phys., 54:3 (2014), 443-461.

[17] С. Д. Глызин, А. Ю. Колесов, Н. Х. Розов, "Неклассические релаксационные колебания в нейродинамике", Модел. и анализ информ. систем, 21:2 (2014), 71-89.

[18] E. M. Izhikevich, "Neural excitability, spiking and bursting", Internat. J. Bifur. Chaos Appl. Sci. Engrg., 10:6 (2000), 1171-1266.

[19] M. I. Rabinovich, P. Varona, A. I. Selverston, H. D. I. Abarbanel, "Dynamical principles in neuroscience", Rev. Modern Phys., 78:4 (2006), 1213-1265.

[20] T. R. Chay, J. Rinzel, "Bursting, beating, and chaos in an excitable membrane model", Biophys. J., 47:3 (1985), 357-366.

[21] J. Rinzel, "A formal classification of bursting mechanisms in excitable systems", Mathematical topics in population biology, morphogenesis and neurosciences (Kyoto, 1985), Lecture Notes in Biomath., 71, Springer, Berlin, 1987, 267-281.

[22] G. B. Ermentrout, N. Kopell, "Parabolic bursting in an excitable system coupled with a slow oscillation", SIAM J. Appl. Math., 46:2 (1986), 233-253.

[23] Bursting. The genesis of rhythm in the nervous system, eds. S. Coombes, P. C. Bressloff, World Scientific, Hackensack, NJ, 2005, xvi+401 pp.

[24] E. M. Izhikevich, "Resonance and selective communication via bursts in neurons having subthreshold oscillations", Biosystems, 67:1-3 (2002), 95-102.

[25] Б. Хэссард, Н. Казаринов, И. Вэн, Теория и приложения бифуркации рождения иикла, Мир, М., 1985, 280 с.; пер. с англ.: В. D. Hassard, N. D. Kazarinoff, Yieh Hei Wan, Theory and applications of Hopf bifurcation, London Math. Soc. Lecture Note Ser., 41, Cambridge Univ. Press, Cambridge-New York, 1981, v+311 pp.

[26] Е.Ф. Мищенко, Ю. С. Колесов, А. Ю. Колесов, Н.Х. Розов, Периодические движения и бифуркационные прочессы в сингулярно возмущенных системах, Физматлит, М., 1995, 336 с.; англ. пер.: E.F. Mishchenko, Yu.S. Kolesov, A. Yu. Kolesov, N. Kh. Rozov, Asymptotic methods in singularly perturbed systems, Monogr. Contemp. Math., Consultants Bureau, New York, 1994, xii+281 pp.

[27] J. J. Hopfield, "Neurons with graded response have collective computational properties like those of two-state neurons", Proc. Nat. Acad. Sci. USA, 81:10 (1984), 3088-3092.

[28] С. Хайкин, Нейронные сети: полный курс, 2-е изд., Изд. дом "Вильямс", М., 2006, 1104 с.; пер. с англ.: S. Haykin, Neural networks. A comprehensive foundation, 2nd ed., Pearson Prentice Hall, Singapore, 1999, 842 pp.

[29] C. M. Marcus, R. M. Westervelt, "Stability of analog neural networks with delay", Phys. Rev. A (3), 39:1 (1989), 347-359.

[30] J. Bélair, "Stability in a model of a delayed neural network", J. Dynam. Differential Equations, 5:4 (1993), 607-623.

[31] J. Bélair, S. A. Campbell, P. van den Driessche, "Frustration, stability and delay-induced oscillations in a neural network model", SIAM J. Appl. Math., 56:1 (1996), 245-255.

[32] K. Gopalsamy, I. Leung, "Delay induced periodicity in a neural netlet of excitation and inhibition", Phys. D, 89:3-4 (1996), 395-426. 
[33] Xiaoxin Liao, Yang Liao, "Stability of Hopfield-type neural networks. II", Sci. China Ser. A, 40:8 (1997), 813-816.

[34] Hui Ye, A. N. Michel, Kaining Wang, "Qualitative analysis of Cohen-Grossberg neural networks with multiple delays", Phys. Rev. E (3), 51:3, part B (1995), 2611-2618.

[35] S. A. Campbell, Shigui Ruan, Junjie Wei, "Qualitative analysis of a neural network model with multiple time delays", Internat. J. Bifur. Chaos Appl. Sci. Engrg., 9:8 (1999), 1585-1595.

[36] P. van den Driessche, Jianhong Wu, Xingfu Zou, "Stabilization role of inhibitory self-connections in a delayed neural network", Phys. D, 150:1-2 (2001), 84-90.

[37] С. Д. Глызин, А. Ю. Колесов, Н. Х. Розов, "Релаксационные автоколебания в сетях Хопфилда с запаздыванием", Изв. РАН. Сер. матем., 77:2 (2013), 53-96; англ. пер.: S. D. Glyzin, A. Yu. Kolesov, N. Kh. Rozov, "Relaxation self-oscillations in Hopfield networks with delay", Izv. Math., 77:2 (2013), 271-312.

[38] А. Ю. Колесов, Н.Х. Розов, Инвариантные торы нелинейных волновых уравнений, Физматлит, М., 2004, 408 с.

[39] Е.Ф. Мищенко, В.А. Садовничий, А. Ю. Колесов, Н. Х. Розов, Автоволновые прочессы в нелинейных средах с диффузией, Физматлит, М., 2005, 432 с.

[40] R. C. Elson, A. I. Selverston, R. Huerta, N. F. Rulkov, M. I. Rabinovich, H. D. I. Abarbanel, "Synchronous behavior of two coupled biological neurons", Phys. Rev. Lett., 81:25 (1998), 5692-5695.

[41] T. J. Lewis, J. Rinzel, "Dynamics of spiking neurons connected by both inhibitory and electrical coupling", J. Comput. Neurosci., 14:3 (2003), 283-309.

[42] N. Kopell, G. B. Ermentrout, "Symmetry and phaselocking in chains of weakly coupled oscillators", Comm. Pure Appl. Math., 39:5 (1986), 623-660.

[43] D. Somers, N. Kopell, "Rapid synchronization through fast threshold modulation", Biol. Cybernet., 68:5 (1993), 393-407.

[44] N. Kopell, D. Somers, "Anti-phase solutions in relaxation oscillators coupled through excitatory interactions", J. Math. Biol., 33:3 (1995), 261-280.

[45] D. Terman, "An introduction to dynamical systems and neuronal dynamics", Tutorials in mathematical biosciences. I. Mathematical neuroscience, Lecture Notes in Math., 1860, Springer-Verlag, Berlin, 2005, 21-68.

[46] С.А. Кащенко, В.В. Майоров, Модели волновой памяти, Книжный дом «ЛИБРОКОМ», М., 2009, 288 с.

[47] G. E. Hutchinson, "Circular causal systems in ecology", Ann. New York Acad. Sci., 50, New York Acad. Sci., New York, NY, 1948, 221-246.

[48] А.Ю. Колесов, Е.Ф. Мищенко, Н.Х. Розов, "Реле с запаздыванием и его $C^{1}$-аппроксимация", Динамические системы и смежные вопросы, Сборник статей. K 60-летию со дня рождения академика Дмитрия Викторовича Аносова, Тр. МИАН, 216, Наука, М., 1997, 126-153; англ. пер.: А. Yu. Kolesov, E. F. Mishchenko, N. Kh. Rozov, "Relay with delay and its $C^{1}$-approximation", Proc. Steklov Inst. Math., 216 (1997), 119-146.

[49] А.Ю. Колесов, Н.Х. Розов, “Автоволновые процессы в цепочках диффузионно связанных уравнений с запаздыванием”, УМH, 67:2(404) (2012), 109-156; англ. пер.: A. Yu. Kolesov, N. Kh. Rozov, "Self-excited wave processes in chains of diffusion-linked delay equations", Russian Math. Surveys, 67:2 (2012), 297-343.

[50] С.Д. Глызин, А. Ю. Колесов, Н.Х. Розов, "Об одном способе математического моделирования химических синапсов", Дифферени. уравнения, 49:10 (2013), 1227-1244; англ. пер.: S. D. Glyzin, A. Yu. Kolesov, N. Kh. Rozov, "On a method for mathematical modeling of chemical synapses", Differ. Equ., 49:10 (2013), 1193-1210.

[51] С. Д. Глызин, А.Ю. Колесов, Н.Х. Розов, "Моделирование эффекта взрыва в нейронных системах", Матем. заметки, 93:5 (2013), 684-701; англ. пер.: 
S. D. Glyzin, A. Yu. Kolesov, N. Kh. Rozov, "Modeling the bursting effect in neuron systems", Math. Notes, 93:5 (2013), 676-690.

[52] А. Ю. Колесов, Е.Ф. Мищенко, Н.Х. Розов, “Новые методы доказательства существования и устойчивости периодических решений в сингулярно возмущенных системах с запаздыванием", Анализ и особенности. Часть 2, Сборник статей. K 70-летию со дня рождения академика Владимира Игоревича Арнольда, Тр. МИАН, 259, Наука, М., 2007, 106-133; англ. пер.: А. Yu. Kolesov, E. F. Mishchenko, N. Kh. Rozov, "New methods for proving the existence and stability of periodic solutions in singularly perturbed delay systems", Proc. Steklov Inst. Math., 259 (2007), 101-127.

[53] С. Д. Глызин, А. Ю. Колесов, Н. Х. Розов, “Экстремальная динамика обобщенного уравнения Хатчинсона”, Журн. вычисл. матем. и матем. физ., 49:1 (2009), 76-89; англ. пер.: S. D. Glyzin, A. Yu. Kolesov, N. Kh. Rozov, "Extremal dynamics of the generalized Hutchinson equation", Comput. Math. Math. Phys., 49:1 (2009), $71-83$.

[54] А. Ю. Колесов, Е.Ф. Мищенко, Н.Х. Розов, Асимптотические методы исследования периодических решений нелинейных гиперболических уравнений, Тр. МИАН, 222, Наука, М., 1998; англ. пер.: А. Yu. Kolesov, E. F. Mishchenko, N. Kh. Rozov, "Asymptotic methods of investigation of periodic solutions of nonlinear hyperbolic equations", Proc. Steklov Inst. Math., 222 (1998), 1-189.

[55] Т. Кохонен, Ассоциативная память, Мир, М., 1980, 240 с.; пер. с англ.: T. Kohonen, Associative memory. A system-theoretical approach, Communication and Cybernetics, 17, Springer-Verlag, Berlin-New York, 1977, ix+176 pp.

[56] С. Д. Глызин, А. Ю. Колесов, Н. Х. Розов, “Дискретные автоволны в нейронных системах", Журн. вычисл. матем. и матем. физ., 52:5 (2012), 840-858; англ. пер.: S.D. Glyzin, A. Yu. Kolesov, N. Kh. Rozov, "Discrete autowaves in neural systems", Comput. Math. Math. Phys., 52:5 (2012), 702-719.

\section{Сергей Дмитриевич Глызин}

Поступила в редакцию

(Sergey D. Glyzin)

05.02 .2015

Ярославский государственный университет

им. П. Г. Демидова

E-mail: glyzin@uniyar.ac.ru

\section{Андрей Юрьевич Колесов (Andrei Yu. Kolesov)}

Ярославский государственный университет

им. П. Г. Демидова

E-mail: kolesov@uniyar.ac.ru

\section{Николай Христович Розов (Nikolai Kh. Rozov)}

Московский государственный университет им. М. В. Ломоносова

E-mail: fpo.mgu@mail.ru 